\title{
CT-PET imaging of lung cancer patients for radiotherapy
}

Citation for published version (APA):

Bosmans, G. H. K. (2007). CT-PET imaging of lung cancer patients for radiotherapy. [Doctoral Thesis, Maastricht University]. Universiteit Maastricht. https://doi.org/10.26481/dis.20071220gb

Document status and date:

Published: 01/01/2007

DOI:

10.26481/dis.20071220gb

Document Version:

Publisher's PDF, also known as Version of record

\section{Please check the document version of this publication:}

- A submitted manuscript is the version of the article upon submission and before peer-review. There can be important differences between the submitted version and the official published version of record.

People interested in the research are advised to contact the author for the final version of the publication, or visit the DOI to the publisher's website.

- The final author version and the galley proof are versions of the publication after peer review.

- The final published version features the final layout of the paper including the volume, issue and page numbers.

Link to publication

\footnotetext{
General rights rights.

- You may freely distribute the URL identifying the publication in the public portal. please follow below link for the End User Agreement:

www.umlib.nl/taverne-license

Take down policy

If you believe that this document breaches copyright please contact us at:

repository@maastrichtuniversity.nl

providing details and we will investigate your claim.
}

Copyright and moral rights for the publications made accessible in the public portal are retained by the authors and/or other copyright owners and it is a condition of accessing publications that users recognise and abide by the legal requirements associated with these

- Users may download and print one copy of any publication from the public portal for the purpose of private study or research.

- You may not further distribute the material or use it for any profit-making activity or commercial gain

If the publication is distributed under the terms of Article $25 \mathrm{fa}$ of the Dutch Copyright Act, indicated by the "Taverne" license above, 
CT-PET imaging of lung cancer patients for radiotherapy 
(C) Copyright G. Bosmans, Maastricht 2007

Druk: Datawyse | Universitaire Pers Maastricht

Cover Design: B. Hanbeukers gebaseerd op een fries van Guus van Eck, die de horizon van Maastricht in al zijn pracht weerspiegelt. 


\title{
CT-PET imaging of lung cancer patients for radiotherapy
}

\author{
Proefschrift \\ ter verkrijging van de graad van doctor \\ aan de Universiteit Maastricht, \\ op gezag van de Rector Magnificus \\ Prof. mr. G.P.M.F. Mols, \\ volgens het besluit van het College van Decanen, \\ in het openbaar te verdedigen, \\ op donderdag 20 december 2007 om 14.00 uur \\ door
}

\section{Geert Bosmans}




\section{Promotor}

Prof. dr. Ph. Lambin

\section{Co-Promotores}

Dr. D.K.M. De Ruysscher

Dr. ir. A.L.A.J. Dekker

\section{Beoordelingscommissie}

Prof. dr. J.M.A. van Engelshoven (voorzitter)

Prof. dr. ir. B.M. ter Haar Romeny (Technische Universiteit Eindhoven)

Prof. dr. S. Senan (Vrije Universiteit Amsterdam)

Prof. dr. G.J.J. Teule

Prof. dr. D. Verellen (Vrije Universiteit Brussel)

Financial support by Siemens Nederland NV for the publication of this thesis is gratefully acknowledged. 


\section{Contents}

Chapter 1 Introduction and outline of the thesis 7

Chapter 2 An "in silico" clinical trial comparing free breathing, slow and respiration correlated computed tomography in lung cancer patients

Radiother Oncol. 2006;81(1):73-80

Chapter 3 Phased attenuation correction in respiration correlated computed tomography/positron emitted tomography Med Phys. 2006;33(6):1840-47

Chapter 4 Respiratory-gated CT as a tool for the simulation of breathing artifacts in PET and PET/CT

Med Phys. Accepted for publication

Chapter 5 Intra-patient variability of tumor volume and tumor motion during conventionally fractionated radiotherapy for locally advanced non-small cell lung cancer: a prospective clinical study Int J Radiat Oncol Biol Phys. 2006;66(3):748-53

Chapter 6 Time trends in the maximal uptake of FDG on PET scan during thoracic radiotherapy. A prospective study in locally advanced non-small cell lung cancer (NSCLC) patients Radiother Oncol. 2007;82(2):145-52

Chapter 7 Time trends in nodal CT volume and nodal motion during radiotherapy for patients with stage III non-small cell lung cancer Int J Radiat Oncol Biol Phys. Accepted for publication 
Samenvatting

Dankwoord

Curriculum Vitae

Publications 


\section{H A P T E R}

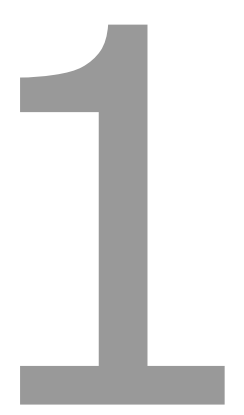

\section{Introduction and outline of the thesis}





\section{CANCER INCIDENCE IN EUROPE AND THE NETHERLANDS}

In 2006, only in Europe more than 3 million cancer cases were estimated to be diagnosed with an overall mortality of about $53 \%$. The total number of new cases of cancer in Europe has increased by 300000 since 2004, and ageing of the population will cause these numbers to continue to increase ${ }^{1}$. It is thus clear that cancer will remain one of the major health problems in Europe for years to come. One of the most common malignancies is lung cancer $(12.1 \%)$ and with an estimated 334800 deaths (19.7\% of total cancer deaths), it is currently the most common cause of death from cancer. The overwhelming majority of these lung cancers are caused by smoking tobacco.

In the Netherlands an average increase of $1.5 \%-2 \%$ new cancer cases is observed each year. In 2005 about 6000 men and 2800 women died of lung cancer. The incidence of lung cancer for men has decreased by one third between 1989 and 2003, but for women the incidence has increased with $80 \%$ in the same period. This can be explained by a change in smoking habits between men and women. The increasingly similar smoking habits of men and women have resulted in a convergence of lung cancer incidence between men and women.

Lung cancer can be subdivided in two main pathological subtypes: Small Cell Lung Cancer (SCLC) and Non-Small Cell Lung Cancer (NSCLC). The majority $(80 \%)$ of patients have NSCLC, a disease only rarely diagnosed at an early stage. Five years after the first diagnosis only $10 \%-15 \%$ of all NSCLC patients are alive (www.ikc.nl), both due to a high incidence of local tumor failure and distant metastases. Research on lung cancer is therefore an important research field aimed at achieving better survival.

\section{Radiotherapy for patients with lung cancer}

Surgery remains the treatment of choice for patients with early stage NSCLC. If patients with stage I-II are medically inoperable because of age or co-morbidity (e.g. poor lung function tests), radiotherapy can be curative. For patients with stage III, often referred as locally advanced NSCLC, combined chemotherapy and radiotherapy has evolved as the standard treatment ${ }^{2-4}$, resulting in an overall 5 year survival of $5 \%-40 \%{ }^{5,6}$.

The two main causes of death in locally advanced NSCLC cancer are distant metastases and lack of local tumor control. Local tumor control with radiotherapy is a function of the total delivered tumor dose in a certain overall treatment time. In addition, improved local control has been shown to yield an improved overall survival ${ }^{7,8}$. Therefore increasing radiation dose or dose escalation could 
lead to better local control and hence improve overall survival ${ }^{9}$. However, dose escalation is hampered by the risk of radiation-induced side effects, like radiation pneumonitis, esophageal damage, spinal cord and heart injury ${ }^{10,}{ }^{11}$. The goal of radiotherapy is to find an optimum between local tumor control and these side effects.

To improve local tumor control, NSCLC patients are increasingly treated with innovative techniques such as highly conformal three-dimensional external beam radiotherapy ${ }^{12}$, Stereotactic Body Radiation Therapy ${ }^{13}$ (SBRT), Intensity modulated radiotherapy ${ }^{14}$ (IMRT), Image Guided Radiotherapy ${ }^{15}$ (IGRT) and in-vivo dosimetry using Electronic Portal Imaging Devices (EPID) ${ }^{16}$, sometimes called as Dose Guided Radiotherapy (DGRT) ${ }^{17}$. To perform these techniques state-of-the-art equipment is used, this means modern linear accelerators equipped with Multi Leaf Collimators (MLC), to shape the radiation beam to the tumor and spare as much as possible the normal tissue, extensive imaging modalities for monitoring the patient's setup in two dimensions (EPID, stereoscopic imaging ${ }^{18}, \ldots$ ) as well as three dimensions (Kilo Voltage or Mega Voltage Cone Beam CT) and possibilities for four-dimensional (4D) radiotherapy (gated radiotherapy ${ }^{19}$ ). Also a Record and Verify system in combination with unfailing digital (DICOM) connections between all used systems is essential to assure a safe patient treatment and to archive and analyze the huge amount of (imaging) data in a systematic central database.

Tumor delineation is one of the weak points of the radiotherapy chain. Large interobserver variations in delineation lung tumors on CT images were reported and especially identification of atelectasis and pathological lymph nodes seems to be difficult ${ }^{20-22}$.

In this thesis two approaches to improve radiotherapy for patients with lung cancer are investigated. The first approach is to better define the target using novel imaging techniques such as 4D-CT and 4D-CT-PET. In the second approach the target is followed during treatment using repeated CT and CT-PET imaging, with the final goal being adaptation of radiotherapy during treatment.

\section{Improving radiotherapy for lung cancer patients by better target definition}

One of the most important critical steps in the radiation treatment process is the definition of the tumor and surrounding tissues. Currently a CT scan is widely used to delineate the gross tumor volume (GTV), which consists of all clinically macroscopic disease, including what is visible on imaging modalities ${ }^{23,} 24$. Safety margins are added to this volume to prevent geographical misses. The clinical target volume (CTV) is created by adding a certain margin to account for microscopic disease. After accurate definition of this GTV and CTV, geo- 
metric uncertainties like set-up errors and tumor motion are covered by an additional safety margin to create the planning target volume (PTV). These geometrical errors can be divided into systematic (every fraction the same) and random (varying from fraction to fraction) deviations from the planned situation. Several margin 'recipes' are described in literature to calculate the CTV-PTV margin ${ }^{25-27}$. Once the target volume is accurately delineated, treatment fields can be defined and the dose distribution can be calculated and optimized.

Optimizing target definition for radiotherapy is therefore extremely important; below are two ways to improve target delineation in lung cancer patients described.

\section{(1) Adding PET to improve GTV delineation}

Computed Tomography (CT) with the current multislice scanners is a fast imaging technique which provides anatomical information. This imaging technique is based on the physical principle of attenuation on an X-Ray fan beam through the patient. A three-dimensional representation of the patient is acquired and different densities are visualized as a grey value with a certain Hounsfield unit. These Hounsfield units can be converted to electron densities which are used to calculate the planned dose distribution. Based on the CT scan, Digitally Reconstructed Radiographs (DRR) which is a computer generated fluoroscopic image, can be created and used as a template for the verification of the patient's position during treatment.

However, CT images are limited to visualize anatomic information only, and they yield no data on intra-tumor heterogeneity. With the advent of Positron Emission Tomography (PET) scanning however, it became possible to visualize and quantify heterogeneities within the tumor. PET is based on the emission of two gamma rays, created by the annihilation of an emitted positron and an electron, which are simultaneous detected. A tracer combined with a radioactive isotope (positron emitter) is injected in the patient. After a rest period, which is needed for the tracer to be diffused within the patient, the patient is imaged. PET images are acquired in several table positions (about $16 \mathrm{~cm}$ ) of several (25) minutes. A whole PET procedure will last between 30-45 minutes. Compared to CT, PET imaging is therefore a slow imaging technique.

The most currently used tracer in PET scanning for radiotherapy is ${ }^{18} \mathrm{~F}$-fluorodeoxyglucose (FDG). This radiotracer is a measure for the rate of glycolysis in the tumor. FDG-PET has a higher sensitivity, specificity and accuracy for detection of tumor involvement than $\mathrm{CT}$ and results in a more accurate staging of malignancies, both for the primary tumor as well as for lymph nodes and distant metastases ${ }^{28}$. FDG-PET, and more specifically the Standardized Uptake Value 
(SUV), can also be very useful for evaluation of treatment response and prediction of recurrence and/or survival after surgery, radiotherapy and chemotherapy 29,30 . It has also been proven to be safe to only irradiate PET positive lymph nodes and thus reducing target volume resulting in a higher tumor dose ${ }^{31-33}$.

Interobserver variation in lung cancer delineation is very large using only CT as imaging information, which could be reduced by standardized institutional protocols ${ }^{20-22,34}$. Delineation accuracy could be further improved by using PET images which also decrease the observer variation ${ }^{35-38}$. An autocontouring method, based on the Source-to-Background ratio in PET images ${ }^{39}$, which was validated in laryngeal carcinomas ${ }^{40}$ was applied in NSCLC patients. With this method we also found a good correlation with pathology, currently the gold standard in defining the tumor, both for the maximal diameter of the primary tumor as well as for the involved lymph nodes ${ }^{41}$. In addition, the delineation variability amongst observers could be reduced even further with the autocontouring method in NSCLC patients.

Incorporating PET imaging in the treatment planning process seems very promising ${ }^{42-44}$ and could for example improve treatment outcome since using this information can lead to sparing of normal tissue and by escalating radiation dose to achieve a higher tumor control probability. Another big advantage of PET imaging is that it can give information on biological characteristics of the tumor and the different areas within the tumor. This might be used in the future to delineate a biological target volume and to adapt radiotherapy treatment. PET imaging is therefore often described as functional or molecular imaging.

Given these benefits, there has been an exponentially growing interest in using (CT-) PET for radiotherapy, as reflected by the number of publications on this subject (Fig. 1).

\section{(2) Adding respiration correlated CT and PET to improve PTV delineation}

One of the major challenges when imaging and treating lung lesions is respiration. The current radiotherapy practice is to account for respiration via a safety margin, usually a population based margin applied to all lung cancer patients. However some lung tumors move more than this margin, resulting in part of the tumor receiving a too low dose (geographic miss) while other tumors barely move, resulting in normal tissue being unnecessarily irradiated. Therefore knowledge of the motion for the individual tumor could optimize radiation therapy. 


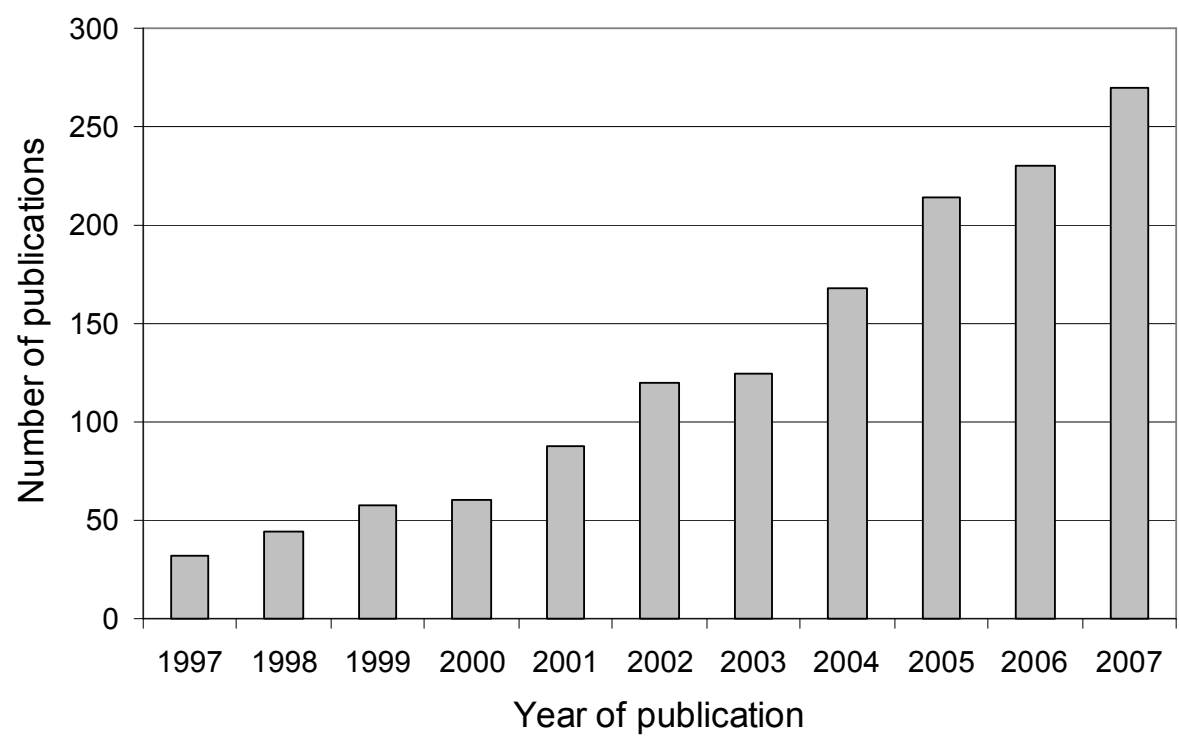

Figure 1: Evolution of the number of publications on PET for radiotherapy. A Medline (www.pubmed.com) search was performed on June 1st 2007 using the terms: [PET AND radiotherapy]. (extrapolated numbers for 2007)

\section{Respiration Correlated CT}

With a fast helical multislice CT scanner the tumor is actually freezed at one location. As this is not necessarily the average tumor position, fast CT scanning can cause a large systematic error. Moreover, it can also lead to distortions of the tumor's shape ${ }^{45}$. Efforts should focus on minimizing these systematic errors, rather than controlling the breathing motion during irradiation (gating) ${ }^{46}$. Several strategies have been investigated to solve these problems when imaging and treating moving lung tumors, such as immobilization of the tumor by Deep Inspiration Breath Hold techniques (DIBH), respiratory gating ${ }^{47}$ or FourDimensional (4D) CT/Respiration Correlated CT (RC-CT) imaging. Other methods are in detail described in a recent report of the AAPM ${ }^{19}$.

RC-CT is an oversampled or low-pitch CT scan during which the respiration signal is recorded. This respiration signal can be measured with different methods, e.g. a thorax band with a pressure sensor ${ }^{48,49}$, infrared markers placed on the patient's chest which is visualized with a camera ${ }^{50}$, measuring the flow of warm (expiration) and cold (inspiration) air with a thermocouple in a mouth mask ${ }^{51}$, magnetic sensors placed on the patients skin ${ }^{52}, \ldots$. Afterwards the CT 
images can be sorted ('binned') according to the respiration phase or respiration amplitude ${ }^{53,54}$. Instead of one CT dataset, several datasets, according to the number of bins, are available for treatment planning. Based on these datasets, movies can be generated in which the motion of the tumor can be measured in all directions.

Incorporating this four-dimensional information into the treatment planning is possible in different ways. Some investigators use Maximum Intensity Projections (MIPs), which reflects the highest data value encountered along the viewing ray for each pixel of volumetric data, giving rise to a full intensity display of the brightest object along each ray on the projection image ${ }^{55}$. Others propose to use only one phase of the respiration, the half-ventilation phase, i.e. the phase where the tumor is at its average position, together with a margin 'recipe' to account for the motion ${ }^{49,56}$.

\section{Respiration correlated CT-PET (RC-CT-PET)}

As mentioned above, a lung tumor can be much better imaged with the combination of PET and CT, than with CT alone. As RC-CT has also shown to be beneficial to image lung cancer, the effect of respiration on PET and the possibility of respiration correlated CT-PET, needs careful consideration in lung cancer.

PET imaging is a slow imaging technique, several minutes are necessary to achieve an acceptable signal/noise ratio. Therefore multiple respiration periods are covered by the images and this obviously blurs the images. This causes artifacts when matching with fast CT images ${ }^{57}$. Some investigators pose that PET is the ideal imaging technique of lung cancer patients because the respiration is already taken into account in the imaging process and the visible lesion represents an Internal Target Volume (ITV) ${ }^{58}$. Many arguments can be raised against this point of view. First, PET images are extremely sensitive to Window/Level settings, therefore automatic delineation software should be developed. Validation of these methods should occur by correlating these PET images with pathology ${ }^{59}$, which is extremely difficult with blurred PET images. Second, accurate information of several parameters, such as the maximal Standardized Uptake Value $\left(\mathrm{SUV}_{\max }\right)$, is not present in classic CT-PET imaging ${ }^{60}$. Moreover, SUV measurements are only reliable when no or little movements of the target are present. Third, attenuation correction could be wrongly performed when combining the fast imaging technique (CT) with the slow one (PET) ${ }^{61}$. Finally, normal CT-PET scanning will represent different intra-tumor heterogeneity due to the respiration, with regard to the tracer uptake. Therefore accurate treatment of these regions, by for example dose painting, is not possible.

Since standard PET procedures, with its respiration blurring, have these limitations in lung cancer, four-dimensional or respiration correlated PET imaging 
have been developed. Similar to RC-CT, these techniques rely on the simultaneous acquisition of list-mode PET data and the respiration signal ${ }^{51,62-64}$.

\section{Improving radiotherapy for lung cancer patients by target adaptation (Adaptive Radiotherapy)}

Nowadays the planned dose distribution is mostly based on a single CT(-PET) scan, taken before the treatment. So the time trends of the tumor are not taken into account in the treatment. Repeated imaging of the tumor during the therapy could give more insight in its dynamics, posing the question if the anatomical and/or biological changes are worthwhile to adapt. A radiation treatment process where the treatment plan can be modified using a systematic feedback of measurements is called 'adaptive radiation therapy' (ART) ${ }^{65}$. With repeated 4D-CT-PET images knowledge is gained about tumor volume (CT), tumor motion (4D) and tumor biological/molecular properties (PET). Several groups are now investigating the time trends of several parameters using different imaging modalities like CT ${ }^{66}$, Mega Voltage CT ${ }^{67,68}$, 4D-CT ${ }^{69,}{ }^{70}$, CT-PET ${ }^{70}$. Haasbeek et al. showed that for their patient population the dosimetric consequences were minimal and therefore adaptive treatment planning was of limited value for fractionated stereotactic radiotherapy ${ }^{71}$. This information about changes during therapy is not only of interest for the primary tumor but also for the involved lymph nodes, since these are also part of the treatment volumes. Until now, this information was never reported.

Since FDG-PET uptake pre-treatment, and more specifically the maximal standardized uptake value $\left(\mathrm{SUV}_{\max }\right)$, is correlated with outcome ${ }^{29,30}$, it is of interest to know the variations of this parameter during treatment. Also an FDG-PET scan post-treatment can predict residual disease and hence outcome in NSCLC patients ${ }^{72,73}$. Therefore, the evolution of the FDG uptake may reflect intrinsic tumor characteristics and might finally be useful to adapt and individualize the treatment early during the course of radiotherapy. This could theoretically be done by adjusting the fractionation scheme or by adding other treatment modalities, such as surgery or drugs.

\section{OBJECTIVES AND OUTLINE OF THE THESIS}

In this thesis two approaches to improve radiotherapy for patients with lung cancer are investigated. The first approach is to better define the target using novel imaging techniques such as respiration correlated (RC-) CT and respiration correlated CT-PET. These techniques take into account the intra-fraction variations and artifacts due to respiration. In the second approach the target is followed during treatment using repeated (RC-) CT and CT-PET imaging, with 
the final goal being adaptation of radiotherapy during treatment and tackle interfraction changes.

In Chapter 2 we investigated how to use the four-dimensional CT information into the treatment planning. More specifically different methods of defining the internal target volume (ITV) were compared with respect to the optimal Clinical Target Volume (CTV) coverage. This was an "in silico" (plannings) study based on real RC-CT patient data.

In Chapter 3 our first results are described with RC-CT-PET imaging with phantom measurements (a lollipop phantom). It mainly concerned the technical feasibility of acquiring RC-CT-PET images and it also investigated differences in uptake using different CTs for the attenuation correction. The purpose of the study was to investigate the difference in uptake between phased attenuation correction and normal attenuation correction.

A similar study was performed in Chapter 4, but now based on real patient data. More different strategies to perform the attenuation correction were studied. Besides the phased attenuation and a fast CT scan we also looked at the average CT data. The influence of tumor motion and tumor location on the SUV recovery was also reported.

Chapter 5 is dedicated to the time trends in tumor volume, tumor motion and breathing frequency of locally advanced lung cancer patients during fractionated radiotherapy. In Chapter 6, we also looked at the changes in biological parameters and more specifically the maximum Standardized Uptake Value $\left(\mathrm{SUV}_{\max }\right)$ between metabolic responders and non-responders during and after radiotherapy, for the same patients as in the previous chapter. In the last Chapter 7, the time trends in volume and motion are described for the involved lymph nodes.

\section{REFERENCES}

1. Ferlay J, Autier P, Boniol M, et al. Estimates of the cancer incidence and mortality in Europe in 2006. Ann Oncol 2007;18:581-592.

2. Senan S, Lagerwaard FJ. The role of radiotherapy in non-small-cell lung cancer. Ann Oncol 2005;16 Suppl 2:ii223-228.

3. Jassem J. The role of radiotherapy in lung cancer: Where is the evidence? Radiother Oncol 2007;83:203-213.

4. Farray D, Mirkovic N, Albain KS. Multimodality therapy for stage III non-small-cell lung cancer. J Clin Oncol 2005;23:3257-3269.

5. Chemotherapy for non-small cell lung cancer. Non-small Cell Lung Cancer Collaborative Group. Cochrane Database Syst Rev 2000:CD002139.

6. Dillman RO, Herndon J, Seagren SL, et al. Improved survival in stage III non-small-cell lung cancer: seven-year follow-up of cancer and leukemia group B (CALGB) 8433 trial. $J$ Natl Cancer Inst 1996;88:1210-1215. 
7. Zatloukal P, Petruzelka L, Zemanova M, et al. Concurrent versus sequential chemoradiotherapy with cisplatin and vinorelbine in locally advanced non-small cell lung cancer: a randomized study. Lung Cancer 2004;46:87-98.

8. Fournel P, Robinet G, Thomas P, et al. Randomized phase III trial of sequential chemoradiotherapy compared with concurrent chemoradiotherapy in locally advanced non-small-cell lung cancer: Groupe Lyon-Saint-Etienne d'Oncologie Thoracique-Groupe Francais de Pneumo-Cancerologie NPC 95-01 Study. J Clin Oncol 2005;23:5910-5917.

9. Arriagada R, Komaki R, Cox JD. Radiation dose escalation in non-small cell carcinoma of the lung. Semin Radiat Oncol 2004;14:287-291.

10. Kong FM, Hayman JA, Griffith KA, et al. Final toxicity results of a radiation-dose escalation study in patients with non-small-cell lung cancer (NSCLC): predictors for radiation pneumonitis and fibrosis. Int J Radiat Oncol Biol Phys 2006;65:1075-1086.

11. Bradley J, Graham MV, Winter K, et al. Toxicity and outcome results of RTOG 9311: a phase I-II dose-escalation study using three-dimensional conformal radiotherapy in patients with inoperable non-small-cell lung carcinoma. Int J Radiat Oncol Biol Phys 2005;61:318328.

12. Senan S, De Ruysscher D, Giraud P, et al. Literature-based recommendations for treatment planning and execution in high-dose radiotherapy for lung cancer. Radiother Oncol 2004;71:139-146.

13. Timmerman RD, Kavanagh BD, Cho LC, et al. Stereotactic body radiation therapy in multiple organ sites. J Clin Oncol 2007;25:947-952.

14. Yom SS, Liao Z, Liu HH, et al. Initial evaluation of treatment-related pneumonitis in advanced-stage non-small-cell lung cancer patients treated with concurrent chemotherapy and intensity-modulated radiotherapy. Int J Radiat Oncol Biol Phys 2007;68:94-102.

15. Dawson LA, Jaffray DA. Advances in image-guided radiation therapy. J Clin Oncol 2007;25:938-946.

16. Nijsten SM, Mijnheer BJ, Dekker AL, et al. Routine individualised patient dosimetry using electronic portal imaging devices. Radiother Oncol 2007;83:65-75.

17. Chen J, Morin O, Aubin M, et al. Dose-guided radiation therapy with megavoltage conebeam CT. Br J Radiol 2006;79 Spec No 1:S87-98.

18. Verellen D, Soete G, Linthout N, et al. Optimal control of set-up margins and internal margins for intra- and extracranial radiotherapy using stereoscopic kilovoltage imaging. Cancer Radiother 2006;10:235-244.

19. Keall PJ, Mageras GS, Balter JM, et al. The management of respiratory motion in radiation oncology report of AAPM Task Group 76. Med Phys 2006;33:3874-3900.

20. Van de Steene J, Linthout N, de Mey J, et al. Definition of gross tumor volume in lung cancer: inter-observer variability. Radiother Oncol 2002;62:37-49.

21. Bowden P, Fisher R, Mac Manus M, et al. Measurement of lung tumor volumes using threedimensional computer planning software. Int J Radiat Oncol Biol Phys 2002;53:566-573.

22. Steenbakkers RJ, Duppen JC, Fitton I, et al. Observer variation in target volume delineation of lung cancer related to radiation oncologist-computer interaction: a 'Big Brother' evaluation. Radiother Oncol 2005;77:182-190.

23. ICRU report 50: Prescribing, recording, and reporting photon beam therapy. Bethesda, MD: International Commission on Radiation Units and Measurements; 1993.

24. ICRU report 62: Prescribing, recording, and reporting photon beam therapy (Supplement to ICRU report 50). Bethesda, MD: International Commission on Radiation Units and Measurements; 1999.

25. Geometric Uncertainties in Radiotherapy - Defining the Planning Target Volume: The British Institute of Radiology; 2003. 
26. Stroom JC, de Boer HC, Huizenga H, et al. Inclusion of geometrical uncertainties in radiotherapy treatment planning by means of coverage probability. Int J Radiat Oncol Biol Phys 1999;43:905-919.

27. van Herk M. Errors and margins in radiotherapy. Semin Radiat Oncol 2004;14:52-64.

28. van Baardwijk A, Baumert BG, Bosmans G, et al. The current status of FDG-PET in tumour volume definition in radiotherapy treatment planning. Cancer Treat Rev 2006;32:245-260.

29. Borst GR, Belderbos JS, Boellaard R, et al. Standardised FDG uptake: a prognostic factor for inoperable non-small cell lung cancer. Eur J Cancer 2005;41:1533-1541.

30. Vansteenkiste JF, Stroobants SG, Dupont PJ, et al. Prognostic importance of the standardized uptake value on (18)F-fluoro-2-deoxy-glucose-positron emission tomography scan in non-small-cell lung cancer: An analysis of 125 cases. Leuven Lung Cancer Group. J Clin Oncol 1999;17:3201-3206.

31. van Der Wel A, Nijsten S, Hochstenbag M, et al. Increased therapeutic ratio by 18FDG-PET CT planning in patients with clinical CT stage N2-N3M0 non-small-cell lung cancer: a modeling study. Int J Radiat Oncol Biol Phys 2005;61:649-655.

32. De Ruysscher D, Wanders S, van Haren E, et al. Selective mediastinal node irradiation based on FDG-PET scan data in patients with non-small-cell lung cancer: a prospective clinical study. Int J Radiat Oncol Biol Phys 2005;62:988-994.

33. De Ruysscher D, Wanders S, Minken A, et al. Effects of radiotherapy planning with a dedicated combined PET-CT-simulator of patients with non-small cell lung cancer on dose limiting normal tissues and radiation dose-escalation: a planning study. Radiother Oncol 2005;77:5-10.

34. Senan S, van Sornsen de Koste J, Samson M, et al. Evaluation of a target contouring protocol for 3D conformal radiotherapy in non-small cell lung cancer. Radiother Oncol 1999;53:247-255.

35. Ashamalla H, Rafla S, Parikh K, et al. The contribution of integrated PET/CT to the evolving definition of treatment volumes in radiation treatment planning in lung cancer. Int J Radiat Oncol Biol Phys 2005;63:1016-1023.

36. Caldwell $\mathrm{CB}$, Mah K, Ung YC, et al. Observer variation in contouring gross tumor volume in patients with poorly defined non-small-cell lung tumors on CT: the impact of 18FDGhybrid PET fusion. Int J Radiat Oncol Biol Phys 2001;51:923-931.

37. Fox JL, Rengan R, O'Meara W, et al. Does registration of PET and planning CT images decrease interobserver and intraobserver variation in delineating tumor volumes for nonsmall-cell lung cancer? Int J Radiat Oncol Biol Phys 2005;62:70-75.

38. Steenbakkers RJ, Duppen JC, Fitton I, et al. Reduction of observer variation using matched CT-PET for lung cancer delineation: a three-dimensional analysis. Int J Radiat Oncol Biol Phys 2006;64:435-448.

39. Daisne JF, Sibomana M, Bol A, et al. Tri-dimensional automatic segmentation of PET volumes based on measured source-to-background ratios: influence of reconstruction algorithms. Radiother Oncol 2003;69:247-250.

40. Daisne JF, Duprez T, Weynand B, et al. Tumor volume in pharyngolaryngeal squamous cell carcinoma: comparison at CT, MR imaging, and FDG PET and validation with surgical specimen. Radiology 2004;233:93-100.

41. van Baardwijk A, Bosmans G, Boersma L, et al. PET-CT-Based Auto-Contouring in NonSmall-Cell Lung Cancer Correlates With Pathology and Reduces Interobserver Variability in the Delineation of the Primary Tumor and Involved Nodal Volumes. Int J Radiat Oncol Biol Phys 2007;68:771-778.

42. Gregoire V, Haustermans K, Geets X, et al. PET-based treatment planning in radiotherapy: a new standard? J Nucl Med 2007;48 Suppl 1:68S-77S. 
43. Grills IS, Yan D, Black QC, et al. Clinical implications of defining the gross tumor volume with combination of CT and 18FDG-positron emission tomography in non-small-cell lung cancer. Int J Radiat Oncol Biol Phys 2007;67:709-719.

44. Nestle U, Kremp S, Grosu AL. Practical integration of [18F]-FDG-PET and PET-CT in the planning of radiotherapy for non-small cell lung cancer (NSCLC): the technical basis, ICRU-target volumes, problems, perspectives. Radiother Oncol 2006;81:209-225.

45. Chen GT, Kung JH, Beaudette KP. Artifacts in computed tomography scanning of moving objects. Semin Radiat Oncol 2004;14:19-26.

46. Engelsman M, Damen EM, De Jaeger K, et al. The effect of breathing and set-up errors on the cumulative dose to a lung tumor. Radiother Oncol 2001;60:95-105.

47. Mageras GS, Yorke E. Deep inspiration breath hold and respiratory gating strategies for reducing organ motion in radiation treatment. Semin Radiat Oncol 2004;14:65-75.

48. Li XA, Stepaniak C, Gore E. Technical and dosimetric aspects of respiratory gating using a pressure-sensor motion monitoring system. Med Phys 2006;33:145-154.

49. Bosmans G, Buijsen J, Dekker A, et al. An "in silico" clinical trial comparing free breathing, slow and respiration correlated computed tomography in lung cancer patients. Radiother Oncol 2006;81:73-80.

50. Ford EC, Mageras GS, Yorke E, et al. Respiration-correlated spiral CT: a method of measuring respiratory-induced anatomic motion for radiation treatment planning. Med Phys 2003;30:88-97.

51. Wolthaus JW, van Herk M, Muller SH, et al. Fusion of respiration-correlated PET and CT scans: correlated lung tumour motion in anatomical and functional scans. Phys Med Biol 2005;50:1569-1583.

52. Remouchamps VM, Huyskens DP, Mertens I, et al. The use of magnetic sensors to monitor moderate deep inspiration breath hold during breast irradiation with dynamic MLC compensators. Radiother Oncol 2007;82:341-348.

53. Lu W, Parikh PJ, Hubenschmidt JP, et al. A comparison between amplitude sorting and phase-angle sorting using external respiratory measurement for 4D CT. Med Phys 2006;33:2964-2974.

54. Abdelnour A, Nehmeh S, Pan T, et al. Phase and amplitude binning for 4D-CT. Phys Med Biol 2007;52:3515-3529.

55. Underberg RW, Lagerwaard FJ, Slotman BJ, et al. Use of maximum intensity projections (MIP) for target volume generation in 4DCT scans for lung cancer. Int J Radiat Oncol Biol Phys 2005;63:253-260.

56. Wolthaus JW, Schneider C, Sonke JJ, et al. Mid-ventilation CT scan construction from fourdimensional respiration-correlated CT scans for radiotherapy planning of lung cancer patients. Int J Radiat Oncol Biol Phys 2006;65:1560-1571.

57. Pan T, Mawlawi O, Nehmeh SA, et al. Attenuation correction of PET images with respiration-averaged CT images in PET/CT. J Nucl Med 2005;46:1481-1487.

58. Caldwell CB, Mah K, Skinner M, et al. Can PET provide the 3D extent of tumor motion for individualized internal target volumes? A phantom study of the limitations of CT and the promise of PET. Int J Radiat Oncol Biol Phys 2003;55:1381-1393.

59. Stroom JC, Blaauwgeers H, van Baardwijk A, et al. The Feasibility of Pathology-correlated Lung Imaging for Accurate Target Definition of Lung Tumors. Int J Radiat Oncol Biol Phys 2007;69:267-275.

60. Nehmeh SA, Erdi YE, Ling CC, et al. Effect of respiratory gating on quantifying PET images of lung cancer. $J$ Nucl Med 2002;43:876-881.

61. Goerres GW, Burger C, Kamel E, et al. Respiration-induced attenuation artifact at PET/CT: technical considerations. Radiology 2003;226:906-910. 


\section{0 | Chapter 1}

62. Nehmeh SA, Erdi YE, Pan T, et al. Quantitation of respiratory motion during 4D-PET/CT acquisition. Med Phys 2004;31:1333-1338.

63. Nehmeh SA, Erdi YE, Pan T, et al. Four-dimensional (4D) PET/CT imaging of the thorax. Med Phys 2004;31:3179-3186.

64. Nagel CC, Bosmans G, Dekker AL, et al. Phased attenuation correction in respiration correlated computed tomography/positron emitted tomography. Med Phys 2006;33:1840-1847.

65. Yan D, Vicini F, Wong J, et al. Adaptive radiation therapy. Phys Med Biol 1997;42:123132.

66. Underberg RW, Lagerwaard FJ, van Tinteren H, et al. Time trends in target volumes for stage I non-small-cell lung cancer after stereotactic radiotherapy. Int J Radiat Oncol Biol Phys 2006;64:1221-1228.

67. Siker ML, Tome WA, Mehta MP. Tumor volume changes on serial imaging with megavoltage CT for non-small-cell lung cancer during intensity-modulated radiotherapy: how reliable, consistent, and meaningful is the effect? Int J Radiat Oncol Biol Phys 2006;66:135141.

68. Kupelian PA, Ramsey C, Meeks SL, et al. Serial megavoltage CT imaging during external beam radiotherapy for non-small-cell lung cancer: observations on tumor regression during treatment. Int J Radiat Oncol Biol Phys 2005;63:1024-1028.

69. Britton KR, Starkschall G, Tucker SL, et al. Assessment of gross tumor volume regression and motion changes during radiotherapy for non-small-cell lung cancer as measured by four-dimensional computed tomography. Int J Radiat Oncol Biol Phys 2007.

70. Bosmans G, van Baardwijk A, Dekker A, et al. Intra-patient variability of tumor volume and tumor motion during conventionally fractionated radiotherapy for locally advanced nonsmall-cell lung cancer: a prospective clinical study. Int J Radiat Oncol Biol Phys 2006;66:748-753.

71. Haasbeek CJ, Lagerwaard FJ, Cuijpers JP, et al. Is adaptive treatment planning required for stereotactic radiotherapy of stage I non-small-cell lung cancer? Int J Radiat Oncol Biol Phys 2007;67:1370-1374.

72. Mac Manus MP, Hicks RJ, Matthews JP, et al. Metabolic (FDG-PET) response after radical radiotherapy/chemoradiotherapy for non-small cell lung cancer correlates with patterns of failure. Lung Cancer 2005;49:95-108.

73. Mac Manus MP, Hicks RJ, Matthews JP, et al. Positron emission tomography is superior to computed tomography scanning for response-assessment after radical radiotherapy or chemoradiotherapy in patients with non-small-cell lung cancer. J Clin Oncol 2003;21:12851292. 


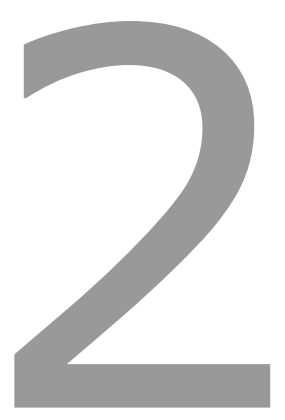

\title{
An "in silico" clinical trial comparing free breathing, slow and respiratory correlated computed tomography in lung cancer patients
}

Radiother Oncol. 2006;81(1):73-80

\author{
Geert Bosmans* \\ Jeroen Buijsen* \\ André Dekker \\ Marije Velders \\ Liesbeth Boersma \\ Dirk De Ruysscher \\ André Minken \\ Philippe Lambin
}

* these authors equally contributed to this study 
22 | Chapter 2

\begin{abstract}
Background and Purpose: To determine which method of internal target volume (ITV) definition based on a respiration correlated CT (RC-CT) allows optimal tumor coverage.

Material and Methods: A free breathing CT $\left(\mathrm{CT}_{\mathrm{fb}}\right)$ and an RC-CT scan were acquired in forty-one lung cancer patients. For twelve patients with a motion $>$ $7 \mathrm{~mm}$ in any direction, a detailed analysis was made. The RC-CT scan was used to measure tumor motion and to reconstruct a $\mathrm{CT}$ at 10 phases $\left(\mathrm{CT}_{10 \mathrm{ph}}\right)$, amongst which the half ventilation $\mathrm{CT}\left(\mathrm{CT}_{\mathrm{hv}}\right)$. By averaging the $\mathrm{CT}_{10 \mathrm{ph}}$, a slow $\mathrm{CT}$ $\left(\mathrm{CT}_{\text {slow }}\right)$ was reconstructed. Based on those scans ITVs were delineated and treatments were planned, where for the ITV $\mathrm{hv}_{\mathrm{hv}}$ an internal margin of (motion amplitude)/4 was used. The treatment plans for the ITVs were projected on the 10 respiration phases. Doses were calculated and averaged over the ten phases to estimate the actual CTV coverage.

Results: The 3D motion was on average $8.1 \mathrm{~mm} \pm 1.0 \mathrm{~mm}(1 \mathrm{SD})$ for all patients; no statistical difference was found between lower and upper lobe tumors. The $\operatorname{ITV}_{\text {slow }}$ was the smallest volume on average $\left(142 \mathrm{~cm}^{3} \pm 38 \mathrm{~cm}^{3}\right)$, followed by the $\operatorname{ITV}_{\mathrm{hv}}\left(160 \mathrm{~cm}^{3} \pm 40 \mathrm{~cm}^{3}\right)$, the $\operatorname{ITV}_{10 \mathrm{ph}}\left(161 \mathrm{~cm}^{3} \pm 41 \mathrm{~cm}^{3}\right)$ and the $\operatorname{ITV}_{\mathrm{fb}}$ $\left(250 \mathrm{~cm}^{3} \pm 63 \mathrm{~cm}^{3}\right)$. Mean CTV doses were between $95 \%$ and $107 \%$ of the prescribed dose for nearly all patients and treatment plans. Analysis of the CTV coverage suggested that underdosage may occur when the $\mathrm{CT}_{\text {slow }}$ is used and a geographical miss occurred using the $\mathrm{CT}_{\mathrm{fb}}$, due to uncorrect localization of the average tumor position.

Conclusions: The $\mathrm{CT}_{\mathrm{hv}}$ seems to be the optimal dataset for delineation, using an adequate anisotropic internal margin of (motion amplitude)/4.
\end{abstract}




\section{INTRODUCTION}

Non-small cell lung cancer (NSCLC) has a poor prognosis. If surgery is not possible, radiation therapy, alone or in combination with chemotherapy, is the principal mode of treatment, resulting in 5 year survival rates of $5 \%-40 \%{ }^{11,17-}$ 19. This poor outcome is due to distant metastases and local recurrences. Since an improved local control (LC) results in a better survival ${ }^{20,22}$, improvement of local treatment is important. One of the strategies to improve LC, is radiation dose escalation.

Dose escalation, however, is associated with an increased risk of complications, especially from damage to the lung and the esophagus. Therefore it is important to define ways to decrease the volume of irradiated normal tissues allowing dose escalation without undue side effects. In our department, we investigated two complementary strategies in lung cancer: 1) better definition of invaded nodes with CT-PET ${ }^{5,24}$ and 2) respiratory correlated CT to individualize the margin needed to account for tumor motion and avoiding geographic misses due to respiration (this paper).

Commonly, the PTV margin is split into a set-up margin, to account for daily set-up and machine variations, and an internal margin to account for tumor motion, i.e. with respiration ${ }^{1}$. Individualizing the internal margin around the tumor may thus tackle two major causes of local failure: first decreasing for some patients irradiated normal tissue volumes thereby allowing dose escalation and second, by decreasing geographical miss.

Respiratory correlated CT (RC-CT) is a new development ${ }^{8,13}$ with which the systematic error (geographical miss) can be minimized and the random error (tumor motion during a radiotherapy fraction) can be estimated for an individual patient. With an RC-CT, separate CTs can be reconstructed from multiple phases of the respiration, allowing estimation of tumor motion as well as the selection of a CT with the tumor in its central position (the half ventilation CT). In our institution, we assume that the tumor moves $7 \mathrm{~mm}$, peak to peak, in all patients. With our known set-up error, standard deviation of $2 \mathrm{~mm}$ both for systematic and random error, and the margin recipe ${ }^{25}$, this assumption results in a $10 \mathrm{~mm}$ total PTV margin in all lung cancer patients (see Appendix). However, it is well known that there is a large variation in tumor motion, ranging from 7 $\mathrm{mm}$ in most patients but much larger amplitudes in some other ${ }^{21,26}$, which illustrates the need for patient-specific measurement of tumor motion and patientspecific PTV margins.

Based on the literature, we hypothesized that the application of RC-CT would offer significant benefits over using free breathing CT by reducing the internal margin but still avoiding geographic misses, thus making radiation dose escalation possible ${ }^{4}$. Furthermore, we expected a benefit of RC-CT over a slow CT ${ }^{15}$, ${ }^{27}$ in the delineation of the tumor. With a slow CT the tumor is smeared out due 
to the breathing motion. Therefore the contrast for tumors located close to the mediastinum or surrounded by atelactic tissue will decrease compared with the non-blurred RC-CT. In this case the delineation will be more difficult and subject to intra- and interobserver variability.

We therefore set up an "in silico" clinical trial in lung cancer patients to compare four treatment plans based on four different datasets: a free breathing CT $\left(\mathrm{CT}_{\mathrm{fb}}\right.$, with standard margins for motion), a slow $\mathrm{CT}$ ( $\mathrm{CT}_{\text {slow }}$, no margins for motion), the half ventilation $\mathrm{CT}$ ( $\mathrm{CT}_{\mathrm{hv}}$, with margins based on measured tumor motion), and the complete 10-phase RC-CT ( $\mathrm{CT}_{10 \mathrm{ph}}$, no margins for motion). The goal of this study was to evaluate which of these four methods results in the best tumor coverage.

\section{MATERIALS AND METHODS}

\section{Patients and CT scans}

Forty-one patients, with stage I to IIIB inoperable non-small cell lung cancer, underwent a free breathing CT immediately followed by an RC-CT scan. The RC-CT scan was performed using offline correlation of a 10-slice spiral CT scan (Siemens Sensation 10, Erlangen, Germany) that was modified to enable a low pitch (minimum 0.1 ), in combination with a respiratory signal obtained by a pressure sensor in a chest belt (AZ-733V, Anzai Medical Corporation, Tokyo, Japan). The raw CT data was reconstructed in 10 phases, from $0 \%$ to $100 \%$ in steps of $10 \%$ of the respiration period starting at end inspiration. In-house developed software with Matlab (Mathworks Inc, Natick, MA) was used to measure the motion of the lesion in all three orthogonal directions by choosing a clearly visible tumor edge and measuring the distance between the extreme positions in an axial, coronal and sagittal movie using the 10 respiratory phases reconstructed from the RC-CT scan. A slow $\mathrm{CT}$ was reconstructed by averaging these 10 phases of the RC-CT.

A detailed analysis was performed on patients with a motion in any direction of more than $7 \mathrm{~mm}$, since we currently use an internal margin based on a motion of $7 \mathrm{~mm}$ (see Appendix). Since the influence of breathing was assumed to be largest for the primary tumor, only the primary tumor was delineated without taking into account the nodal status.

All patients gave written informed consent before entering this study, which was approved by the Medical Ethical Committee according to the Dutch law. 


\section{Target Volume Delineation}

The internal target volume (ITV) was defined as the clinical target volume (CTV) with margins for tumor motion based on the ICRU $62{ }^{1}$.

We used four different methods to determine the margin for internal motion, resulting in four ITVs:

1. Free breathing $\mathrm{CT}$ : On the $\mathrm{CT}_{\mathrm{fb}}$, the gross tumor volume (GTV) was delineated with a window/level setting of 1700/-300 Hounsfield Units (HU), and a $5 \mathrm{~mm}$ margin for microscopic disease was added to obtain the clinical target volume (CTV). For the internal target volume (ITV), an $8 \mathrm{~mm}$ internal margin in all directions was added to the CTV to account for internal motion of the tumor (see Appendix). This was performed using commercial delineation software (Focal 4.2.0, CMS, St. Louis, USA). This ITV will later be referred to as ITV $_{\mathrm{fb}}$.

2. Slow CT: On the $\mathrm{CT}_{\text {slow, }}$ the tumor was smeared out, and the resulting visible structure was delineated with a window/level setting of 1700/$300 \mathrm{HU}$. After adding the $5 \mathrm{~mm}$ margin to the GTV for microscopic disease, this was assumed to reflect the ITV $\mathrm{Vlow}_{\text {slow }}$

3. Half ventilation CT: With in-house built software, the motion of the tumor in 3 orthogonal directions was measured. Thereafter the CT from the phase with the tumor in its central position (half ventilation CT $=$ $\mathrm{CT}_{\mathrm{hv}}$ ) was selected and exported to the delineation software. On this $\mathrm{CT}_{\mathrm{hv}}$, the GTV and CTV (= GTV $\left.+5 \mathrm{~mm}\right)$ were delineated. To obtain the $\mathrm{ITV}_{\mathrm{hv}}$, the CTV was expanded with an asymmetric margin of $\mathrm{A} / 4$, with A being the peak-to-peak amplitude of tumor motion in each of the three orthogonal directions (see Appendix for an explanation of this internal margin).

4. Ten-phase CT: In the same in-house built software, a volume was drawn around the moving visible tumor. This volume was termed the internal gross tumor volume (IGTV). To get the ITV $\mathrm{I0ph}_{10 \mathrm{p}}$, $5 \mathrm{~mm}$ margin was added to the IGTV for microscopic disease. Finally, this ITV $_{10 \mathrm{ph}}$ was projected on the slow $\mathrm{CT}$ and exported to the delineation software.

\section{Influence of tumor motion on CTV coverage}

To compare the influence of tumor motion on the actually delivered dose in the CTV for the four delineation methods, we chose to ignore set-up uncertainties. Therefore, treatment plans were made for the four different ITVs (XiO version 4.2.0, CMS, St. Louis, USA) using a multigrid superposition algorithm. To be able to compare the four methods, the same number of beams and similar gantry angles were used and only field blocking, weight and/or wedge angle were different between the four plans. Criteria for the planning were a dose of $70.2 \mathrm{~Gy}$ 
$(39 \times 1.8 \mathrm{~Gy}, \mathrm{BID})$ to the ITV (acceptable inhomogeneity 95\% - 107\%) and a spinal cord dose below 55 Gy.

To estimate the actually delivered dose, the plans were projected on all the ten phases of the RC-CT. The CTV was contoured on all phases by delineating the visible tumor and 3D-expanding this volume with $5 \mathrm{~mm}$ for microscopic disease. For each phase the dose volume histogram (DVH) of the CTV was calculated, and the average dose distribution was calculated over all ten phases, for each of the four ITV treatment plans. From these DVHs, the minimal dose and the mean dose in the CTV was recorded.

\section{Statistical analysis}

All results are expressed as mean \pm standard error of the mean (SEM). Statistical differences between paired parameters from treatment plans based on $\mathrm{CT}_{\mathrm{fb}}$ versus $\mathrm{CT}_{\text {slow }}, \mathrm{CT}_{\mathrm{hv}}$ and $\mathrm{CT}_{10 \mathrm{ph}}$ were evaluated using the Wilcoxon signed rank test. The statistical difference in tumor motion between the different tumor locations were evaluated using a student t-test. Differences were considered to be significant if the 2-tailed p-value was less than 0.05 .

\section{RESULTS}

\section{Patient characteristics}

The 3D vector motion of all 41 patients is displayed in Fig. 1. Two different groups were identified according to their location, upper lobe tumors $(n=27)$ versus lower lobe tumors $(\mathrm{n}=14)$. The average $3 \mathrm{D}$ motion for the entire group was $8.1 \mathrm{~mm} \pm 1.0 \mathrm{~mm}(1 \mathrm{SD})$. There was no statistically significant difference of tumor motion between the tumor locations $(\mathrm{p}=0.136)$, although the average motion was somewhat bigger for lower lobe tumors, on average $10.1 \mathrm{~mm} \pm 2.1$ $\mathrm{mm}$, compared with the upper lobe tumors, on average $7.0 \mathrm{~mm} \pm 1.0 \mathrm{~mm}$. Fifteen patients $(36.5 \%)$ showed a tumor motion $>7 \mathrm{~mm}$ in any direction. Due to technical reasons we could not complete a full analysis for three of these 15 patients. In the remaining 12 patients we performed a more detailed analysis (Table 1). As stated in the methods section, patients with $\mathrm{N}+$ disease $(8 / 12)$ were planned as if they had no metastatic lymph nodes (N0). 


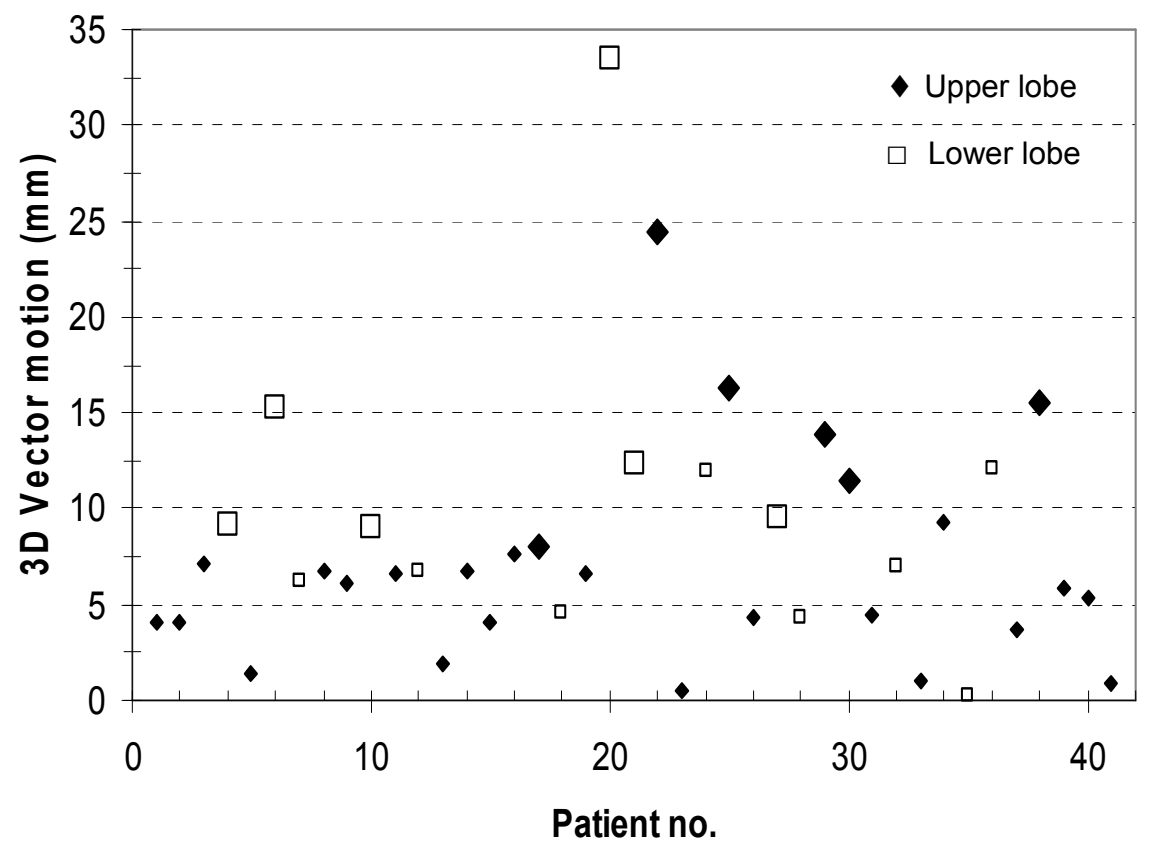

Figure 1: Overview of the 3D vector motion of all 41 patients for different tumor locations. The big markers indicate the patients which were studied more in detail and had an individual motion $>7 \mathrm{~mm}$ in any direction.

Table 1: Patient characteristics of the 12 patients with a tumor motion in any direction $>7 \mathrm{~mm}$. The GTV size of the primary tumor was measured on the free breathing CT.

\begin{tabular}{|c|c|c|c|l|l|}
\hline Patient no. & Age & Gender & Stage & GTV Size $\left(\mathrm{cm}^{3}\right)$ & Location \\
\hline 1 & 74 & F & T2N0M0 & 64.9 & Right upper lobe \\
2 & 56 & M & T1N3M0 & 0.4 & Left upper lobe \\
3 & 79 & M & T2N0M0 & 65.0 & Right lower lobe \\
4 & 79 & M & T2N2M0 & 50.9 & Right upper lobe \\
5 & 79 & M & T4N0M0 & 12.2 & Right lower lobe \\
6 & 58 & F & T2N2M0 & 272.2 & Right upper lobe \\
7 & 79 & M & T4N2M0 & 33.5 & Right lower lobe \\
8 & 60 & M & T1N0M0 & 5.0 & Left lower lobe \\
9 & 52 & F & T2N3M0 & 79.0 & Left lower lobe \\
10 & 71 & M & T4N2M0 & 7.3 & Right upper lobe \\
11 & 61 & F & T2N2M0 & 101.9 & Right upper lobe \\
12 & 60 & M & T1N3M0 & 7.5 & Right upper lobe \\
\hline
\end{tabular}




\section{Tumor motion}

For the 12 patients with a tumor motion $>7 \mathrm{~mm}$ in any direction, the mean amplitude for tumor motion was $5.0 \mathrm{~mm} \pm 1.5 \mathrm{~mm}$ (1 SD) in anterior-posterior (AP), $12.5 \mathrm{~mm} \pm 1.7 \mathrm{~mm}$ in craniocaudal (CC) and $3.1 \mathrm{~mm} \pm 1.1 \mathrm{~mm}$ in lateral (LR) direction (Table 2). The maximum craniocaudal motion was $28.5 \mathrm{~mm}$. The quadratic sum of the motion, i.e. the $3 \mathrm{D}$ vector motion, was $14.5 \mathrm{~mm} \pm 2.1 \mathrm{~mm}$.

\begin{tabular}{|c|c|c|c|c|}
\hline Patient no. & $\mathrm{AP}$ & $\mathrm{CC}$ & LR & Vector \\
\hline 1 & 9.8 & 28.5 & 14.7 & 33.5 \\
\hline 2 & 16.6 & 18 & 0 & 24.5 \\
\hline 3 & 1 & 15 & 3 & 15.3 \\
\hline 4 & 3 & 15 & 2.9 & 15.6 \\
\hline 5 & 0 & 12 & 2.9 & 12.3 \\
\hline 6 & 10.8 & 12 & 2.4 & 16.3 \\
\hline 7 & 0 & 9 & 0 & 9.0 \\
\hline 8 & 2 & 9 & 0 & 9.2 \\
\hline 9 & 2.5 & 9 & 2 & 9.6 \\
\hline 10 & 8,8 & 9 & 5.9 & 13.9 \\
\hline 11 & 6.4 & 9 & 3 & 11.4 \\
\hline 12 & 2.5 & 7.4 & 2 & 8.1 \\
\hline MEAN & 5.0 & 12.5 & 3.1 & 14.5 \\
\hline SEM & 1.5 & 1.7 & 1.1 & 2.1 \\
\hline
\end{tabular}

\section{ITVs for the four different methods}

As shown in Fig. 2, the ITVs based on the $\mathrm{CT}_{\text {slow }}$, the $\mathrm{CT}_{\mathrm{hv}}$ and the $\mathrm{CT}_{10 \mathrm{ph}}$ were all considerably smaller than the ITV based on the $\mathrm{CT}_{\mathrm{fb}}(\mathrm{p}=0.002, \mathrm{p}=0.002$ and $\mathrm{p}=0.002$ respectively). On average, the ITV obtained from the $\mathrm{CT}_{\text {slow }}$ was the smallest $\left(49 \% \pm 6 \%\right.$ of the ITV $\left._{\mathrm{fb}}\right)$, the ITV from the $\mathrm{CT}_{10 \mathrm{ph}}$ and the ITV from the $\mathrm{CT}_{\mathrm{hv}}$ were comparable, $57 \% \pm 6 \%$ and $59 \% \pm 6 \%$ of the $\mathrm{ITV}_{\mathrm{fb}}$ respectively. 


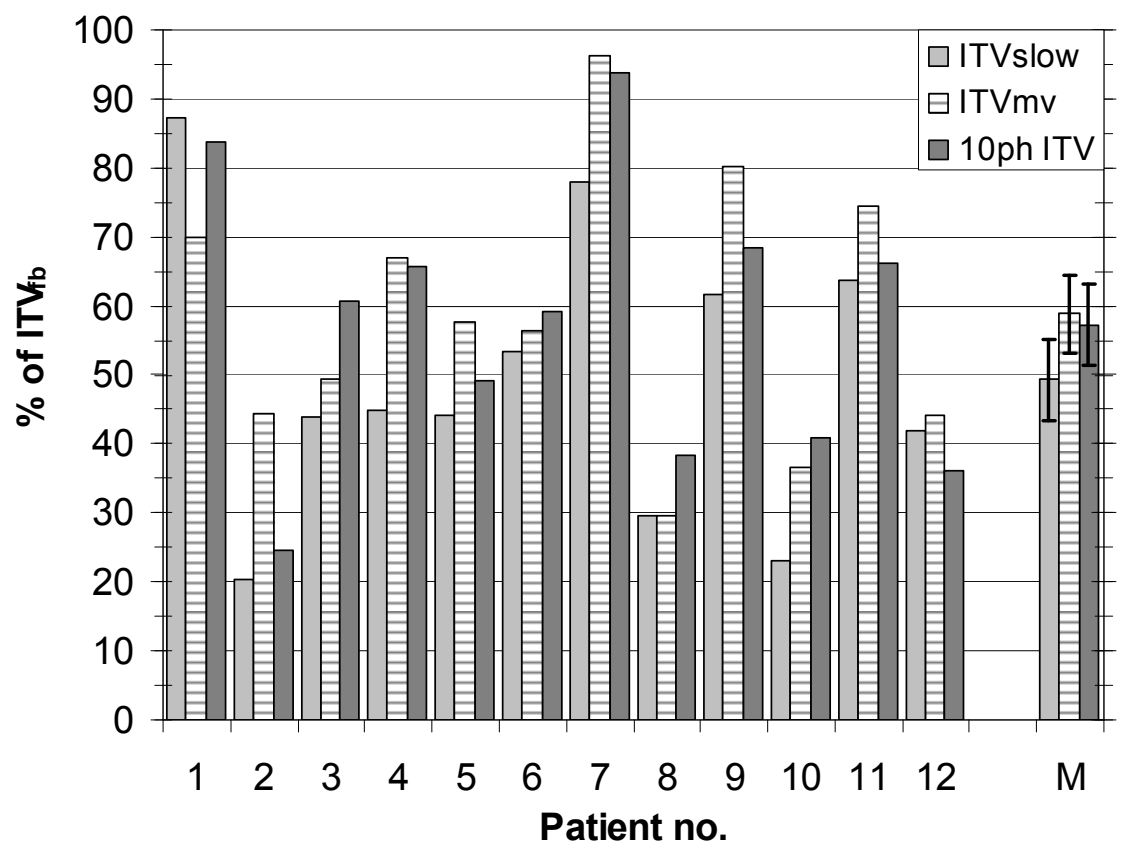

Figure 2: Internal target volumes for four different delineation methods in 12 patients. Volumes are shown relative to the free breathing Internal Target Volume $\left(\mathrm{ITV}_{\mathrm{fb}}\right)$. On average $(\mathrm{M})$ the volumes, ITV slow $_{\text {ITV }}$, and ITV I0ph $_{10}$, can be reduced to about $50 \%$. Error bars on the average values show the Standard Error of the Mean.

\section{CTV coverage}

The CTV coverage for treatment plans based on the ITVs for the four different delineation methods for all 12 patients is shown in Fig. 3. The CTVs drawn for each technique were projected on all the 10 respiratory phases and the mean dose to the CTV was calculated $(100 \%=70.2$ Gy). The mean CTV doses resulting from the 3 alternative plans were not significantly different from the conventional free breathing plan. Almost all mean CTV doses were between $95 \%$ and $107 \%$ of 70.2 Gy, suggesting that the margins used were adequate. When looking at the minimum doses per respiration phase, we found that in one patient in one phase the treatment plan based on the $\mathrm{CT}_{\text {slow }}$ and in one patient the treatment plan based on the $\mathrm{CT}_{\mathrm{fb}}$ did not reach the $95 \%$ minimum dose requirement. 

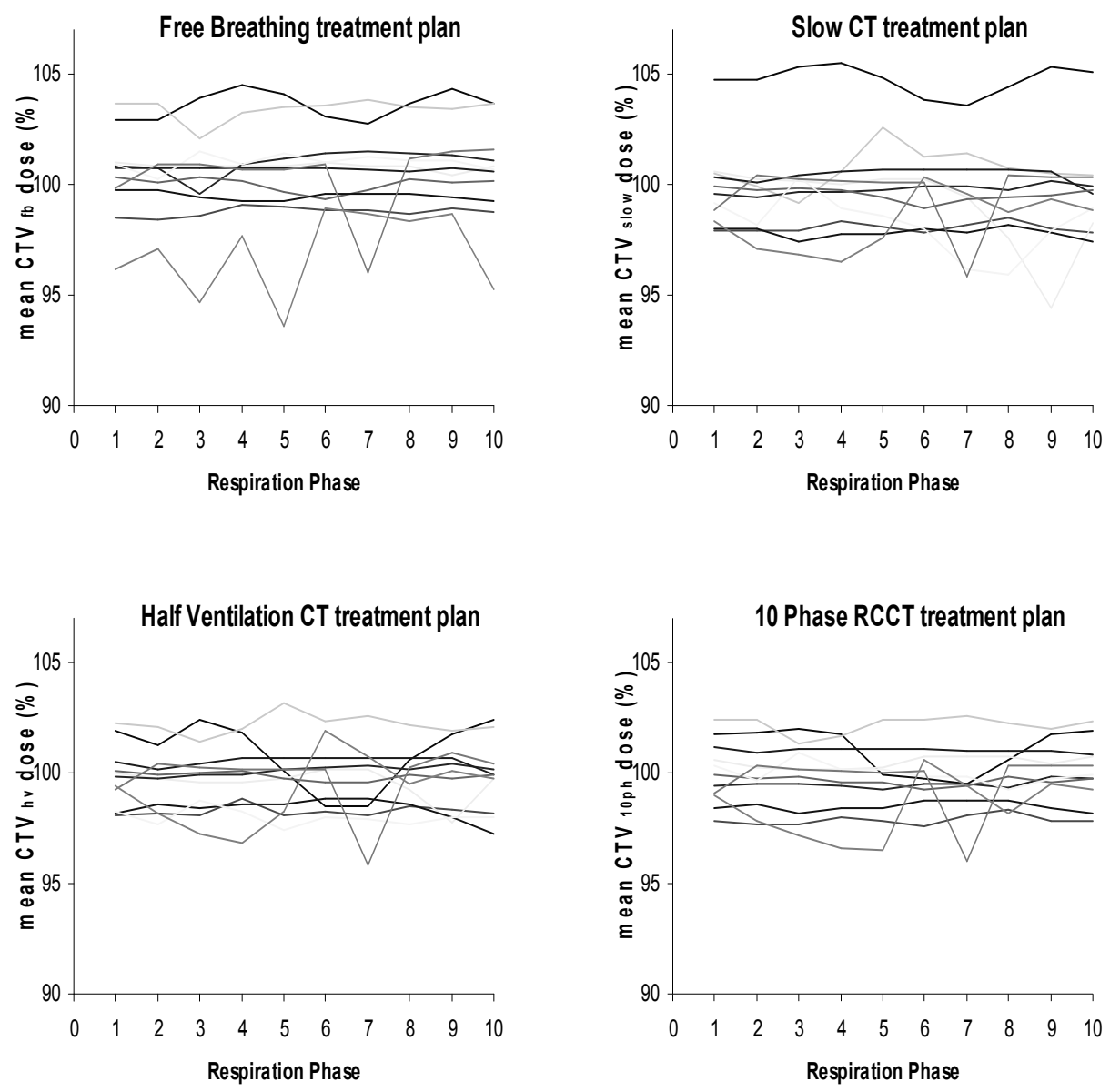

Figure 3: CTV coverage for four different treatment plans in 12 patients. The CTVs delineated on the free breathing scan $\left(\mathrm{CTV}_{\mathrm{fb}}\right)$, on the slow $\mathrm{CT}\left(\mathrm{CTV}_{\text {slow }}\right)$, the half ventilation $\mathrm{CT}$ scan $\left(\mathrm{CTV}_{\mathrm{hv}}\right)$ and the 10 phases of the RC-CT $\left(\mathrm{CTV}_{10 \mathrm{ph}}\right)$ were projected on all 10 phases. For each phase of the respiration and for each technique the mean CTV dose was calculated, where $100 \%$ equals 70.2 Gy. In almost all patients good CTV coverage is achieved in all phases for all the four different treatment plans. Only in one patient in one phase the treatment plan based on the slow CT plan, the CTV did not reach the $95 \%$ dose requirement, and in one patient in two phases in de $\mathrm{CT}_{\mathrm{fb}}$ ).

Although the mean CTV doses were thus adequate, localized underdosage in the CTV may occur, since the volumes were drastically reduced with the respiration correlated techniques compared to the conventional $\mathrm{CT}_{\mathrm{fb}}$. Detailed analysis of the minimum doses in all datasets showed that there were several patients who showed a significant underdosage using the $\mathrm{CT}_{\text {slow }}$ for treatment planning. To study this issue in more detail, a 3-point moving average was calculated for all minimal doses (for example for phase 2 the mean of the minimum doses of 
phases 1, 2 and 3 was calculated). Assuming that cold spots in consecutive phases were located in the same area of the tumor, using the 3-point moving average, a dose less than $83 \%$ in three consecutive phases meant that it was likely there was a real coldspot, since a dose less than $83 \%$ in three phases cannot be compensated by normal doses in other phases $((3 \times 83 \%+7 \times 100 \%)$ $/ 10 \approx 95 \%$. In Fig. 4, the 3-point moving average is shown for the minima in the $\mathrm{CTV}$. For several patients it was shown that the $\mathrm{CT}_{\text {slow }}$ plan results in underdosage.
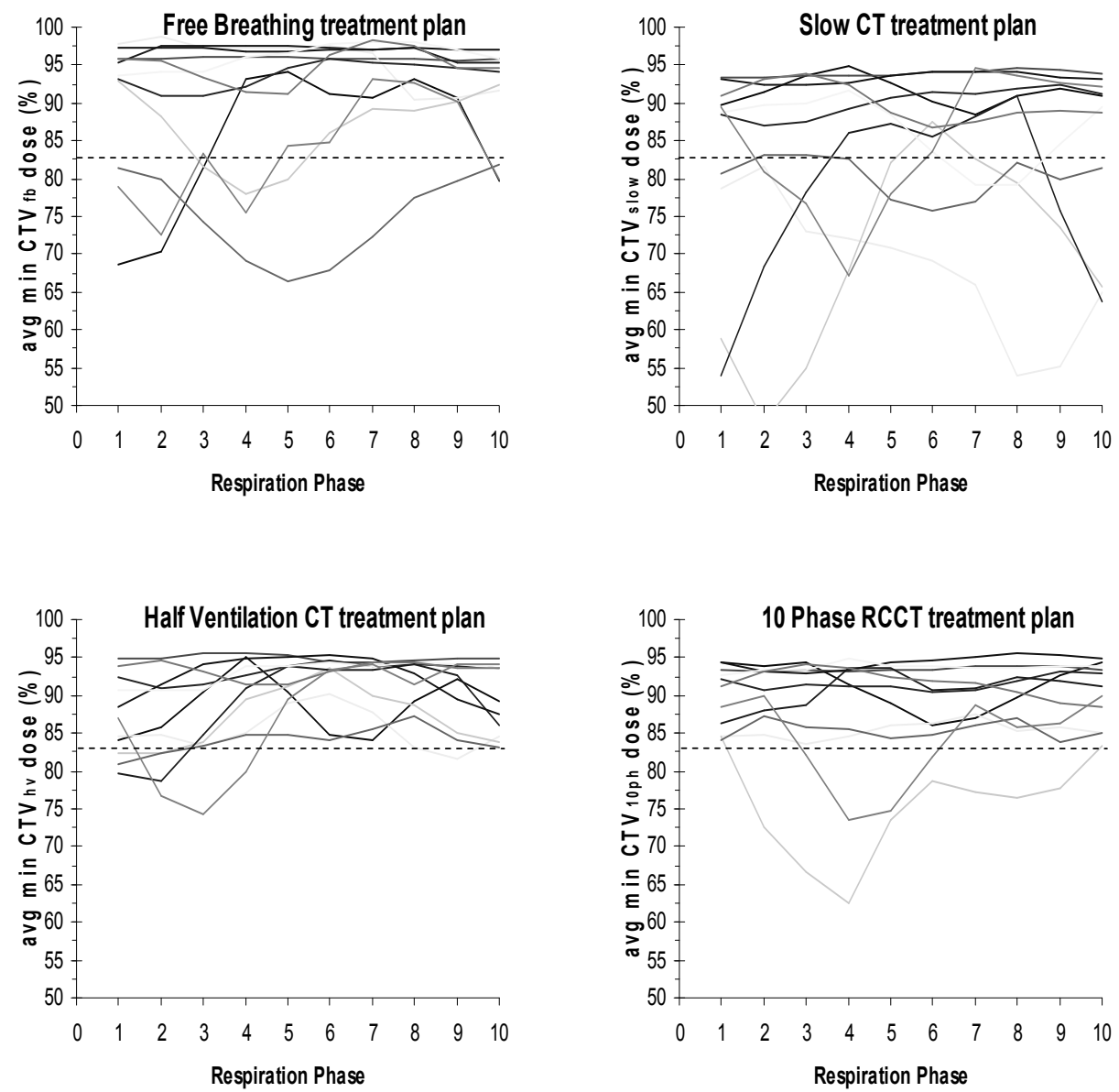

Figure 4: Representation of possible underdosage of four different treatment plans for 12 patients. The average minimum CTV dose of three adjacent respiration phases are shown for the treatment plans based on the $\mathrm{CT}_{\mathrm{fb}}$, the $\mathrm{CT}_{\text {slow }}$, the $\mathrm{CT}_{\text {hv }}$ and the $\mathrm{CT}_{10 \mathrm{ph}}$. For several patients the $\mathrm{CT}_{\text {slow }}$ treatment plan shows for almost every respiration phase a dose less then $83 \%$, which strongly suggests a real underdosage. The underdosage in the treatment plan based on the $\mathrm{CT}_{\mathrm{fb}}$ was due to geographical miss. 
A possible explanation of this underdosage is shown in Fig. 5, where one Beams Eye View (BEV) of the treatment plan based on the $\mathrm{CT}_{\text {slow }}$ scan for a patient is projected on all 10 respiratory phases. It is clearly visible that for some phases the CTV is not adequately covered by the beam settings caudally.
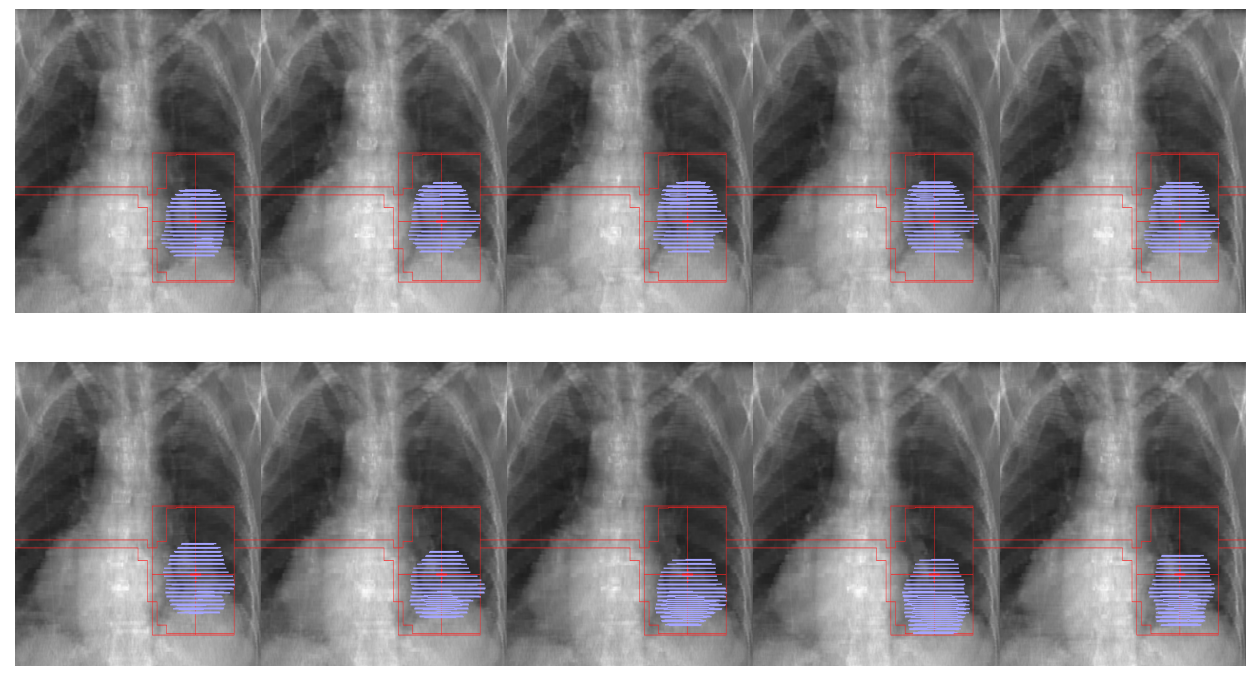

Figure 5: Projection of one (gantry $=0^{\circ}$ ) beam of the treatment plan based on the $\mathrm{CT}_{\text {slow }}$ scan for the patient with a cranial caudal motion of $15 \mathrm{~mm}$, on all ten phases of the respiration. In blue the CTV is shown on all ten phases. It is clearly shown that in some phases the tumor is not adequately covered by the beam settings.

The underdosage for one patient using the treatment plan based on the $\mathrm{CT}_{10 \mathrm{ph}}$ can be explained by the difficult delineation of the IGTV for this patient without using positron emission tomography information. For some patients also the treatment plan based on the $\mathrm{CT}_{\mathrm{fb}}$ showed underdosage, caused by the fact that the position of the isocentre, defined as the centre of mass of the $\mathrm{ITV}_{\mathrm{fb}}$, was located close to end inspiration instead of half ventilation, thereby causing a "geographical miss". In Fig. 6, the craniocaudal trajectory is shown for the patient with the largest motion, with the position of the isocentre of the different treatment plans indicated.

\section{DISCUSSION}

The internal margin due to respiration has two main contributors in CT based radiotherapy planning. First, $\mathrm{CT}$ is a fast imaging technique that captures the 
tumor at only one phase of the respiration ${ }^{2}$. If the tumor is not at its central position when it is imaged, a systematic error is introduced possibly resulting in a geographic miss ${ }^{6}$. Second, the motion of the tumor with respiration causes an intra-fractional random error, which cannot be estimated on a single CT. The systematic error has a much larger contribution to the internal margin than the random error ${ }^{25}$.

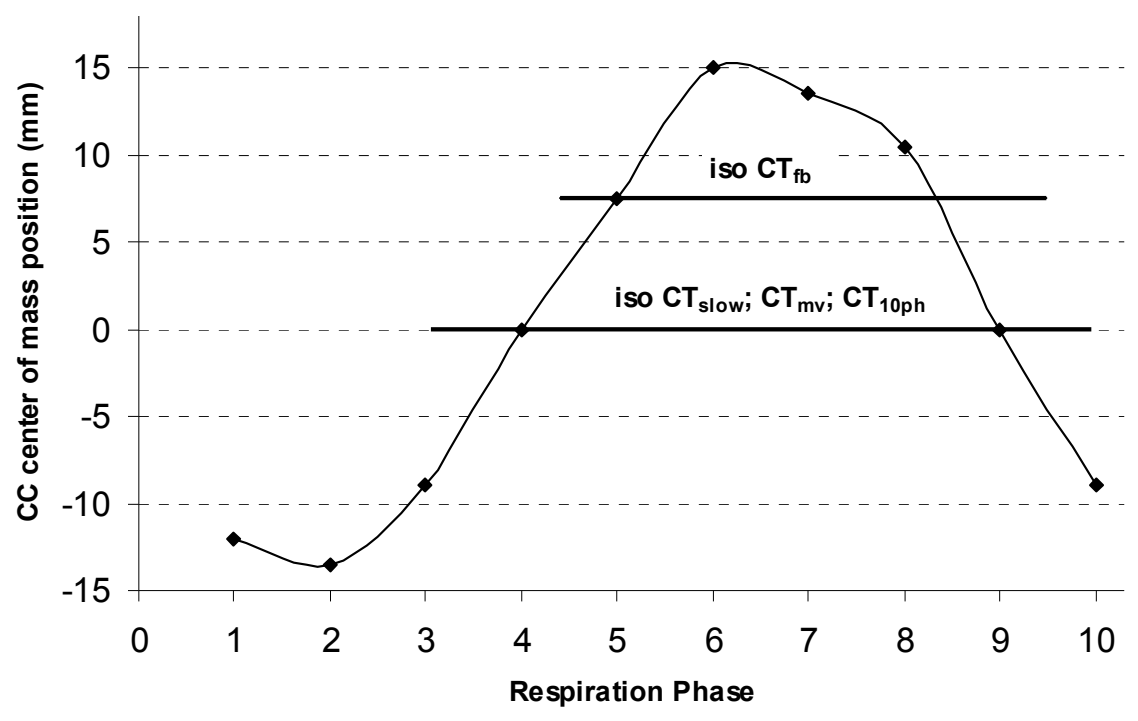

Figure 6: Craniocaudal trajectory of the center of mass of the tumor in one patient with a geographical miss. The locations of the isocenter of all treatment plans are indicated.

Respiration correlated CT (RC-CT) scans give information about tumor motion during respiration. Including this information in treatment planning may lead to more optimal treatment plans, avoiding geographical miss and minimizing margins. We showed that by using RC-CT the ITVs were significantly smaller than ITVs delineated in the $\mathrm{CT}_{\mathrm{fb}}$, suggesting that considerable dose escalation might be possible. However, analysis of the CTV coverage (see further) suggested that underdosage may occur when the ITV $\mathrm{hv}_{\mathrm{hv}}$ is not used, such that the $\mathrm{CT}_{\mathrm{hv}}$ seems to be the optimal dataset for delineation.

\section{Tumor delineation}

We used the RC-CT information to determine the ITV with three different methods, the $\mathrm{CT}_{\text {slow }}$, the $\mathrm{CT}_{\mathrm{hv}}$ and the $\mathrm{CT}_{10 \mathrm{ph}}$. The smallest volume was obtained using the $\mathrm{CT}_{\text {slow }}$ scan. This can be explained by the "smear out effect", which 
plays an important role using the $\mathrm{CT}_{\text {slow }}$. Regions where the tumor stays for very short time may therefore show a lower density and less contrast, and may therefore not be recognized as tumor. Therefore, the GTV delineation is very dependent on Window/Level settings. Especially the most cranial and caudal parts of the motion path can be missed. Furthermore, particularly in slow CT images, it is sometimes difficult to distinguish tumor from infiltrate.

The delineation of the ITV $\mathrm{I0ph}_{1}$ for all the different phases of the respiratory cycle was labour-intensive. Although other authors also suggested using only a few phases to determine the ITV, this remains time-consuming and is more subject to variations in delineations ${ }^{12}$.

First results, reported by Hugo et al., showed that the mean position (half ventilation) during the treatment course and the breathing pattern remains stable, supporting the use of the half ventilation scan for tumor delineation ${ }^{10}$. In addition, in an earlier study we showed that the tumor motion does not change significantly during a course of radiotherapy ${ }^{3}$.

\section{Internal margins and systematic errors}

The internal margin due to respiration applied to the half ventilation CT, A/4 (with A the peak-to-peak amplitude), is quite tight, in comparison with other authors $^{7,25}$. However, the projection of these plans on all the respiratory phases did not show any underdosage, thus this margin recipe for internal motions seems to be valid. The average motion observed in our study was comparable with other studies using CT scans to evaluate tumor motion ${ }^{2,16}$. In addition, we could not found a significant difference in tumor motion depending on the tumor location, which is in agreement with several other authors ${ }^{26,27}$.

There was a difference in average tumor position between the different treatment techniques based on the RC-CT and the $\mathrm{CT}_{\mathrm{fb}}$ scan for some patients. This resulted in a geographical miss when using the $\mathrm{CT}_{\mathrm{fb}}$. For patients with a smaller motion $(<7 \mathrm{~mm})$ of the lesion this effect would of course be less pronounced and will probably not be clinically relevant because this systematic error will be covered by additional setup errors.

\section{Tumor coverage}

The next step was to study the influence of tumor motion on the CTV coverage for the four different treatment plans. For this purpose, we estimated the actually delivered dose distribution by projecting the treatment plans on all 10 phases of the RC-CT, and by averaging the dose distributions over the 10 phases. This part of the study was difficult, since due to limitations of the planning system it was not possible to summarize the dose volume histograms (DVH) of the different breathing phases. The mean doses in the different CTVs 
were not significantly different between the treatment plans, although for one patient the 95\% mean CTV dose was not reached (Fig. 4) for the treatment plan based on the $\mathrm{CT}_{\mathrm{fb}}$ and the $\mathrm{CT}_{\text {slow. }}$ The minimum doses were more difficult to interpret, because the planning system only gave information about the absolute minimum dose, but it does not provide information about the location of this minimum. Therefore, it is possible that a cold spot (Dose $<95 \%$ ) observed in one phase of the respiratory cycle is compensated by a higher dose (Dose $>$ $100 \%$ ) in the same voxel in another phase.

The minimal dose data were analyzed in more detail with the use of a 3-point moving average. Using these strict criteria, in several patients a real underdosage was find when using the $\mathrm{CT}_{\text {slow }}$. This would give evidence for the benefical effect of gated treatment for patients with a large tumor motion, like stated in other articles ${ }^{23}$. However, it has to be realized that the analysis of CTV coverage was carried out for treatment plans based on the ITVs. The advantage of this choice was that we could separately analyze the effect of tumor motion on CTV coverage. However, it cannot be excluded that when repeating this analysis of CTV coverage for PTV plans, this would probably show less underdosage.

In conclusion, smaller internal target volumes could be reached using information about breathing and tumor motion. Although the $\mathrm{CT}_{\text {slow }}$ reduced the ITV the most, the delineation is inaccurate due to the blurring of the lesion at the edges. In addition, the CTV coverage analysis suggested that use of the $\mathrm{CT}_{\text {slow }}$ for delineation might result in underdosage. Therefore we propose that the treatment plan based on the half ventilation $\mathrm{CT}$, combined with the individual anisotropic margins, is the most suitable method for delineation of lung tumors. This method is easy (only one phase), the tumor is clearly visible (no blurring due to motion) and the reduced margin is adequate.

\section{APPENDIX: CALCULATION OF INTERNAL MARGINS}

Internal margins can be calculated using the margin recipe proposed by van Herk ${ }^{25}$. The total margin $\left(M_{P T V}\right)$ can individually be calculated by the following formula:

$$
M_{P T V}=2.5 \sqrt{\sum_{\text {setup }}^{2}+\sum_{\text {internal }}^{2}}+0.7 \sqrt{\sigma_{\text {setup }}^{2}+\sigma_{\text {int ernal }}^{2}}
$$

With $\sum_{\text {setup }}$ and $\sum_{\text {internal }}$ being the standard deviation of the systematic error for setup and organ motion respectively, $\sigma_{\text {setup }}$ and $\sigma_{\text {internal }}$ the standard deviation of the random error for setup and organ motion respectively. 
Assuming a sinusoidal tumor motion with peak-to-peak amplitude of $\mathrm{A}$, the internal margin can be calculated as follows:

$$
\Sigma_{\text {internal }}=\sigma_{\text {internal }}=\frac{A}{2 \sqrt{2}}
$$

Using this in Equation A1 gives:

$$
\begin{aligned}
& \sum_{\text {total }}=\sqrt{\sum_{\text {setup }}^{2}+\sum_{\text {internal }}^{2}}=\sqrt{\sum_{\text {setup }}^{2}+A^{2} / 8} \\
& \sigma_{\text {total }}=\sqrt{\sigma_{\text {setup }}^{2}+\sigma_{\text {internal }}^{2}}=\sqrt{\sigma_{\text {setup }}^{2}+A^{2} / 8}
\end{aligned}
$$

When using a total PTV margin of $10 \mathrm{~mm}$, and using our known set-up error from our institute, i.e. a standard deviation of $2 \mathrm{~mm}$ both for systematic and random errors based on EPID measurements, we assumed a tumor motion of 7 $\mathrm{mm}$. The setup errors have been calculated in a previous group of lung cancer patients with the same immobilization method. Since this is the major source of setup uncertainties we could extrapolate these results to our patient group For a tumor moving $7 \mathrm{~mm}$ with respiration, the margin recipe thus prescribes an internal margin of $8 \mathrm{~mm}$ for a free breathing CT based plan (setup errors excluded).

In half ventilation CT the systematic error is zero, while the random error $\sigma_{\text {internal }}$ of a tumor with motion $A$ is again $A / 2 \sqrt{2}$. Applying the margin recipe yields an internal margin of $0.7 \mathrm{~A} / \sqrt{ } 8$ or approximately $\mathrm{A} / 4$.

\section{REFERENCES}

1. ICRU report 62: Prescribing, recording, and reporting photon beam therapy (Supplement to ICRU report 50). Bethesda, MD: International Commission on Radiation Units and Measurements; 1999.

2. Allen AM, Siracuse KM, Hayman JA, Balter JM. Evaluation of the influence of breathing on the movement and modeling of lung tumors. Int J Radiat Oncol Biol Phys 2004;58:12511257.

3. Bosmans G, van Baardwijk A, Dekker A, et al. Intra-patient variability of tumor volume and tumor motion during conventionally fractionated radiotherapy for locally advanced nonsmall cell lung cancer: A Prospective Clinical Study. Int J Rad Onc Biol Phys 2006; 66(3):748-53.

4. Cho BC, Craig T. More optimal dose distributions for moving lung tumours: a planning study. Radiother Oncol 2006;79:122-130.

5. De Ruysscher D, Wanders S, van Haren E, et al. Selective mediastinal node irradiation based on FDG-PET scan data in patients with non-small-cell lung cancer: a prospective clinical study. Int J Radiat Oncol Biol Phys 2005;62:988-994. 
6. Engelsman M, Damen EM, De Jaeger K, van Ingen KM, Mijnheer BJ. The effect of breathing and set-up errors on the cumulative dose to a lung tumor. Radiother Oncol 2001;60:95105.

7. Engelsman M, Sharp GC, Bortfeld T, Onimaru R, Shirato H. How much margin reduction is possible through gating or breath hold? Phys Med Biol 2005;50:477-490.

8. Ford EC, Mageras GS, Yorke E, Ling CC. Respiration-correlated spiral CT: a method of measuring respiratory-induced anatomic motion for radiation treatment planning. Med Phys 2003;30:88-97.

9. Fowler JF, Tome WA, Fenwick JD, Mehta MP. A challenge to traditional radiation oncology. Int J Radiat Oncol Biol Phys 2004;60:1241-1256.

10. Hugo G, Vargas C, Liang J, Kestin L, Wong JW, Yan D. Changes in the respiratory pattern during radiotherapy for cancer in the lung. Radiother Oncol 2006;78:326-331.

11. Jeremic B, Classen J, Bamberg M. Radiotherapy alone in technically operable, medically inoperable, early-stage (I/II) non-small-cell lung cancer. Int J Radiat Oncol Biol Phys 2002;54:119-130.

12. Jin JY, Ajlouni M, Chen Q, Yin FF, Movsas B. A technique of using gated-CT images to determine internal target volume (ITV) for fractionated stereotactic lung radiotherapy. $R a-$ diother Oncol 2006;78:177-184.

13. Keall PJ, Starkschall G, Shukla H, et al. Acquiring 4D thoracic CT scans using a multislice helical method. Phys Med Biol 2004;49:2053-2067.

14. Kwa SL, Lebesque JV, Theuws JC, et al. Radiation pneumonitis as a function of mean lung dose: an analysis of pooled data of 540 patients. Int J Radiat Oncol Biol Phys 1998;42:1-9.

15. Lagerwaard FJ, Van Sornsen de Koste JR, Nijssen-Visser MR, et al. Multiple "slow" CT scans for incorporating lung tumor mobility in radiotherapy planning. Int $J$ Radiat Oncol Biol Phys 2001;51:932-937.

16. Mageras GS, Pevsner A, Yorke ED, et al. Measurement of lung tumor motion using respiration-correlated CT. Int J Radiat Oncol Biol Phys 2004;60:933-941.

17. Marino P, Preatoni A, Cantoni A. Randomized trials of radiotherapy alone versus combined chemotherapy and radiotherapy in stages IIIa and IIIb nonsmall cell lung cancer. A metaanalysis. Cancer 1995;76:593-601.

18. Perez CA, Pajak TF, Rubin P, et al. Long-term observations of the patterns of failure in patients with unresectable non-oat cell carcinoma of the lung treated with definitive radiotherapy. Report by the Radiation Therapy Oncology Group. Cancer 1987;59:1874-1881.

19. Pritchard RS, Anthony SP. Chemotherapy plus radiotherapy compared with radiotherapy alone in the treatment of locally advanced, unresectable, non-small-cell lung cancer. A meta-analysis. Ann Intern Med 1996;125:723-729.

20. Schaake-Koning C, van den Bogaert W, Dalesio O, et al. Effects of concomitant cisplatin and radiotherapy on inoperable non-small-cell lung cancer. N Engl J Med 1992;326:524530 .

21. Shimizu S, Shirato H, Kagei K, et al. Impact of respiratory movement on the computed tomographic images of small lung tumors in three-dimensional (3D) radiotherapy. Int J Radiat Oncol Biol Phys 2000;46:1127-1133.

22. Sibley GS, Jamieson TA, Marks LB, Anscher MS, Prosnitz LR. Radiotherapy alone for medically inoperable stage I non-small-cell lung cancer: the Duke experience. Int J Radiat Oncol Biol Phys 1998;40:149-154.

23. Underberg RW, Lagerwaard FJ, Slotman BJ, Cuijpers JP, Senan S. Benefit of respirationgated stereotactic radiotherapy for stage I lung cancer: an analysis of 4DCT datasets. Int J Radiat Oncol Biol Phys 2005;62:554-560. 


\section{8 | Chapter 2}

24. van Der Wel A, Nijsten S, Hochstenbag M, et al. Increased therapeutic ratio by 18FDG-PET CT planning in patients with clinical CT stage N2-N3M0 non-small-cell lung cancer: a modeling study. Int J Radiat Oncol Biol Phys 2005;61:649-655.

25. van Herk M. Errors and margins in radiotherapy. Semin Radiat Oncol 2004;14:52-64.

26. van Sornsen de Koste JR, Lagerwaard FJ, Nijssen-Visser MR, Graveland WJ, Senan S. Tumor location cannot predict the mobility of lung tumors: a 3D analysis of data generated from multiple CT scans. Int J Radiat Oncol Biol Phys 2003;56:348-354.

27. Wurstbauer K, Deutschmann H, Kopp P, Sedlmayer F. Radiotherapy planning for lung cancer: slow CTs allow the drawing of tighter margins. Radiother Oncol 2005;75:165-170. 


\title{
C H A P T E R
}

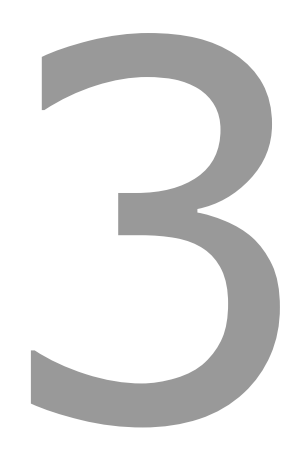

\section{Phased attenuation correction in respiration correlated CT-PET}

Med Phys. 2006;33(6):1840-7

\author{
Cathryn Nagel* \\ Geert Bosmans* \\ André Dekker \\ Michel Öllers \\ Dirk De Ruysscher \\ Philippe Lambin \\ André Minken \\ Norbert Lang \\ Klaus Schäfers
}

* these authors equally contributed to this study 


\section{ABSTRACT}

Purpose: Motion of lung tumors with respiration causes difficulties in the imaging with CT and PET. Since accurate knowledge of the position of the tumor and the surrounding tissues is needed for radiation treatment planning it is important to improve CT-PET image acquisition. The purpose of this study was to evaluate the potential to improve image acquisition using phased attenuation correction in respiration correlated CT-PET, where data of both modalities was binned retrospectively.

Methods and materials: Respiration correlated scans were made on a Siemens Biograph Sensation 16 CT-PET scanner which was modified to make a low pitch CT scan and list-mode PET scan possible. A lollipop phantom was used in the experiments. The sphere with a diameter of $3.1 \mathrm{~cm}$ was filled with approximately $20 \mathrm{MBq}{ }^{18} \mathrm{~F}$-FDG. Three longitudinal movement amplitudes were tested: $2.5,3.9$ and $4.8 \mathrm{~cm}$. After collection of the raw CT data, list-mode PET data, and the respiratory signal CT-PET images were binned to ten phases with help of in-house built software. Each PET phase was corrected for attenuation with CT data of the corresponding phase. For comparison, the attenuation correction was also performed with non-respiration correlated (non-RC) CT data. The volume and the amplitude of the movement were calculated for every phase of both the CT and PET data (with phased attenuation correction). Maximum and average activity concentrations were compared between the phased and non-phased attenuation corrected PET.

Results: With a standard, non-RC CT-PET scan, the volume was underestimated by as much as $46 \%$ in CT and the PET volume was overestimated to $370 \%$. The volumes found with RC-CT-PET scanning had average deviations of $1.9 \%( \pm 4.8 \%)$ and $1.5 \%( \pm 3.4 \%)$ from the actual volume, for the CT and PET volumes respectively.

Evaluation of the maximum activity concentration showed a clear displacement in the images with non-RC attenuation correction, and activity values were on average $14 \%( \pm 12 \%)$ lower than with phased attenuation correction. The standard deviation of the maximum activity values found in the different phases was a factor 10 smaller when phased attenuation correction was applied.

Conclusion: In this phantom study we have shown that a combination of respiration correlated CT-PET scanning with application of phased attenuation correction can improve the imaging of moving objects and can lead to improved volume estimation and a more precise localization and quantification of the activity. 


\section{INTRODUCTION}

The combination of CT and PET scanning is the best way to image lung tumors for radiotherapy ${ }^{1,2}$. However, with the integration of the two modalities in one machine problems may occur because CT-PET combines a fast multi-slice computed tomography (CT) technique with the much slower positron emitted tomography (PET), which should be considered when imaging lung tumors that may move up to $2 \mathrm{~cm}$ (peak-to-peak amplitude) with respiration ${ }^{3}$. A multi-slice CT scan of the thorax is completed in a few seconds and a tumor might be recorded in less than a second. Given the respiration cycle length (3-6 seconds) a moving tumor will most likely not be imaged at its average position in a multislice CT scan. The measurement of a single bed position with PET on the other hand takes about 5 minutes, and will represent several full respiratory cycles. On a PET image a moving tumor will thus be smeared out around its average position.

When the tumor movement is considerable these shortcomings of the two separate imaging modalities can lead to a distortion of the combined CT-PET image ${ }^{4-7}$ resulting in an underestimation of the measured standardized uptake value ${ }^{8}$ (SUV, which is a prognostic factor in lung cancer ${ }^{9}$ ) or a mislocalization of the tumor. Therefore, in radiotherapy, an isotropic margin of usually $15 \mathrm{~mm}$ is added to the tumor volume that is visible on CT-PET images to account for microscopic disease and for the possible mislocalization of the tumor on the CTPET image and for tumor movement during radiation. Depending on the actual movement of the tumor this margin may be too small or too large leading to an under-dosage of the tumor or an over-dosage of the normal tissue, respectively.

To reduce the influence of movement on tumor location several new techniques have been developed. One of these involves correlation of the respiration signal to the scan data. This technique has previously been studied and shows good results for both $\mathrm{CT}^{10,11}$ and $\mathrm{PET}{ }^{12,13}$. In a respiration correlated CT scan (RC$\mathrm{CT})$ the respiration signal is recorded simultaneously with the acquisition of the CT data. This data is then binned retrospectively according to the respiration phase during which it was acquired. This provides information on the tumor position for each phase of the respiration.

Previous studies with 4D-CT-PET in patients ${ }^{14,15}$ show good results in reducing motion artifacts and increasing maximum SUV. In these studies however, the PET data is acquired in a gated mode and binned prospectively into 10 fractions of, for example, $500 \mathrm{~ms}$. Because the bins have predefined time lengths problems may arise in the case of irregular breathing patterns.

The respiration correlated PET (RC-PET) scanning in the protocol proposed in this study uses continuous acquisition of the data in list-mode and phase binning is carried out retrospectively. In retrospective binning the time length of the bins 
is determined individually for each period of the respiration. This way the binning can take into account a varying breathing frequency.

In a CT-PET scanner attenuation correction of PET can be performed automatically with information obtained from the fast CT scan. This CT image represents the position of the lung tumor and surrounding tissues during only part of the respiratory cycle, however, while the PET image contains the information of several full-breathing cycles. The CT image used to correct for attenuation then not fully corresponds to the PET image, thereby introducing motion artifacts ${ }^{16}$. After binning the CT and uncorrected PET data into corresponding phases, the tumor and tissue positions on PET and CT match more closely. Therefore an additional advantage of phase binning is the possible reduction of motion artifacts introduced to the PET scan during CT-based attenuation correction. Applying phase correlated attenuation correction will thus, most likely, lead to a more accurate correction and fewer introductions of motion artifacts. A great advantage of an improved attenuation correction would be the possibility of auto-segmentation for delineation of tumors based on the SUV.

For radiotherapy of lung cancer patients the ultimate goal is to adapt the margins needed in delineation of the tumor to the actual movement of the tumor in each patient. The aim of the current study is to show the potential of respiration correlation of PET with retrospective binning and of phased attenuation correction in RC-CT-PET scanning.

\section{METHODS AND MATERIALS}

Scans were made on a Siemens Biograph Sensation 16 CT-PET scanner (Siemens Medical Solutions, Hoffmann Estates, IL, USA). For the 16-slice spiral CT scan the following settings were used; $0.125 \mathrm{pitch}, 3.0 \mathrm{~mm} / \mathrm{s}$ table feed, 0.5 s rotation time, $16 \times 1.5 \mathrm{~mm}$ scanning slice thickness, $3 \mathrm{~mm}$ reconstructed slice thickness, $500 \mathrm{~mm}$ transaxial field-of-view. The PET data was obtained in listmode using a modified list-mode research package originally provided by Siemens. List-mode data were acquired for 10 minutes, retrospectively binned according to the respiration cycle and reconstructed with $3 \mathrm{~mm}$ reconstructed slice thickness. 


\section{Phantom}

For analysis of the accuracy of respiration correlated PET scanning in general and phased attenuation correction in particular, a so-called lollipop phantom was used (Fig. 1).

The sphere at the end of the rod was filled with approximately $20 \mathrm{MBq}{ }^{18} \mathrm{~F}$ fluoro-deoxyglucose (FDG). A sphere with a diameter of approximately $3.2 \mathrm{~cm}$ and a volume of $16.0 \mathrm{cc}$ is used (Model ECT/HS/ST, Data Spectrum Corporation (DSC), Hillsborough, NC, USA). The positions 1 to 5 (see Fig. 1a) can be varied in order to get different movement amplitudes. The sphere moved parallel to the cranial-caudal axis. Three longitudinal peak-to-peak amplitude settings were tested of $2.5,3.9$ and $4.8 \mathrm{~cm}$, respectively. The rod was mounted on a disc, which is powered by a motor. The frequency of the motion was set to approximately 18 rotations per minute, corresponding to $0.3 \mathrm{~Hz}$. This is comparable to the average respiration frequency of humans.

The respiration signal was measured with two systems; the first system was synchronized with the CT on time, the second with the PET on time. For CT synchronization the Anzai system (AZ-733V, Anzai Medical Corporation, Tokyo, Japan) was used. This consists of a pressure load cell that is fitted into a chest belt. The signal for the PET data was acquired with a soft, foam-filled sensor (Graseby, UK) connected via a tube to an air pressure detector (BioVET monitoring system, Spin Systems, Brisbane, Australia). This system is based on the flow in the tube caused by pressure on the foam, and not like the Anzai system on the pressure itself.

The belt, in which both cells are fitted, was wrapped around the bottom plate of the phantom and connected to the rod through a rubber band as shown in Fig. $1 b$.

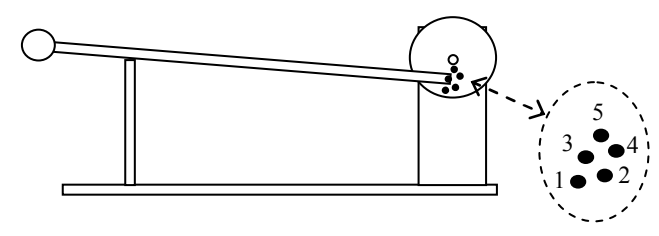

a)

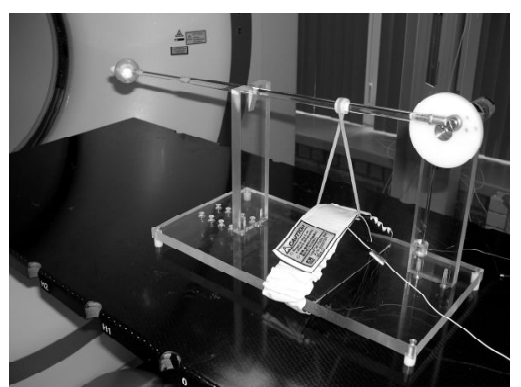

Figure 1: The lollipop phantom; a) schematically and b) actual. 
The maximum movement amplitude found for lung tumors is about $2 \mathrm{~cm}$ with an average of $12 \mathrm{~mm}$ in cranial-caudal direction ${ }^{3}$. The sensitivity of the Anzai, however, was not suitable for measurement of amplitudes smaller than the 2.5 $\mathrm{cm}$ tested in this study.

For comparison purposes regular non-RC-CT and PET scans were also made. To show the advantage of phased attenuation correction, correction of the phased PET data was also performed with the non-RC-CT scan.

Due to a scanner problem no non-respiratory correlated CT-PET scan was made for the sphere moving with an amplitude of $4.8 \mathrm{~cm}$.

\section{Phase binning}

\section{$C T$}

After acquisition, the CT data was binned to ten phases according to the respiration phase during which it was recorded. The respiration signal was synchronized with the CT data by recording the CT on time. Phase binning was performed with a modified version of a software package that was provided by Siemens. Each period was divided into ten bins of $10 \%$ each, where phase one was chosen to start at the maximum amplitude.

\section{PET}

Phase binning was also performed retrospectively. A trigger could be set at an arbitrary amplitude of the respiratory signal. The trigger signal was used for retrospective sorting of the raw list-mode data into 10 phase bins. The binning was, like the binning of CT data, based on phase only and not on the amplitude. The trigger, and thus phase one, was again chosen to start at the maximum amplitude of the signal.

\section{Attenuation correction}

The protocol proposed here required attenuation correction to be applied outside the scanner. Software was therefore developed in-house (Matlab, Mathworks Inc, Natick, MA, USA). The attenuation correction process as described by Kinahan et al. ${ }^{17,18}$ was followed. This correction is based on the following formula:

$$
\int_{-\infty}^{+\infty} f(x, y) d y^{\prime}=e^{\int_{-\infty}^{+\infty} \mu(x, y) d y^{\prime}} \bullet p\left(x^{\prime}, \phi\right)
$$


where the term on the left represents the actual distribution of FDG in the patient, the exponential term stands for the attenuation along the line of response (LOR) at detector position $x^{\prime}(x, y)$ and projection angle $\phi(x, y)$, and $p\left(x^{\prime}, \phi\right)$ is the measured signal.

An overview of the steps in the attenuation correction process is given in Fig. 2.

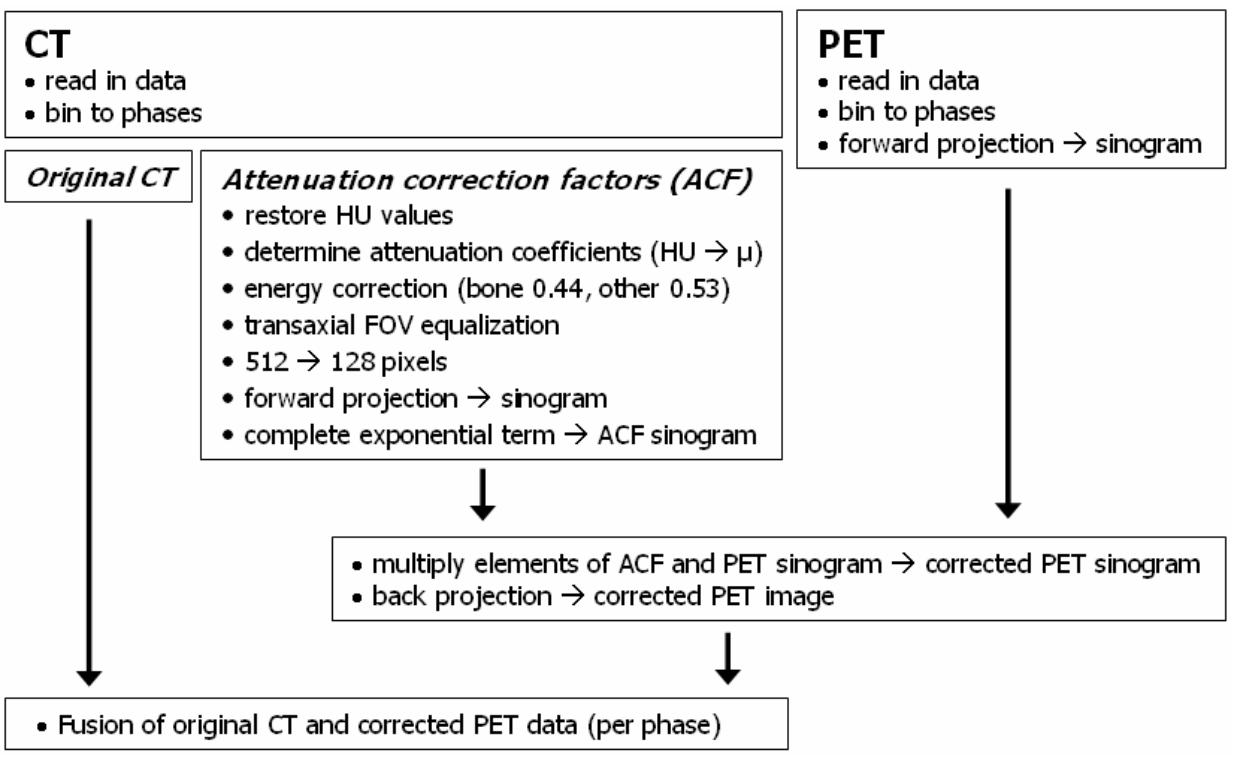

Figure 2: Overview of the steps in the attenuation correction process.

After the field of view (FOV) and number of pixels have been equalized the map of attenuation correction factors (ACF) can be obtained through forward projection. With forward projection a sinogram is constructed. This consists of the integrals along the LORs. The attenuation correction factors in the sinogram then need to be multiplied by the pixel size to account for the path length. Taking the exponent completed the correction factors.

The raw PET data of the corresponding slice and phase is also converted to a sinogram. If the PET and CT images have matching FOV and the same number of pixels, each element of the PET sinogram has a corresponding element in the ACF sinogram. Multiplying each element of the PET sinogram with the value of the corresponding ACF element will perform the attenuation correction. The corrected PET sinogram can then be converted back to an image through back projection or iterative image reconstruction (OSEM).

The procedure discussed here was applied to the data of every slice and every phase. 


\section{Analysis}

Maximum activity concentration values in each of the phases obtained with the phased and non-RC attenuation correction were determined and the percentage deviation per phase was calculated.

The accuracy of the respiration correlation was determined by comparing the movement amplitude of the spheres on the PET data to the motion found with RC-CT and to the real amplitude. For the measurements on the CT and PET images, the absolute vertical position of the spheres (centre) was calculated on sagittal images for each of the ten phases. The maximum displacement was calculated as the distance of the minimum and maximum sphere position.

The volumes of the spheres on the PET and CT images were compared to the volume of the actual sphere. This analysis was used to determine both the accuracy of the respiration correlation as well as that of the phased attenuation correction. The PET volume was determined using a 34\% threshold in the region of interest, as was determined with in-house experiments following the methods described by Erdi et al. ${ }^{19}$ and Daisne et al. ${ }^{20}$. The maximum activity concentration $(100 \%)$ was determined by averaging the nine highest values found within all the phases. This average was taken to account for the influence of extreme values.

The sphere volumes in CT images were determined after manual delineation of the sphere with window width and window level settings of 1000 and 0 , respectively.

\section{RESULTS}

Fig. 3 gives an example of the phased PET (bottom) and CT (top) images of the experiment with a $2.5 \mathrm{~cm}$ amplitude.

In Fig. 4 two images of the same slice and phase of a PET scan from the experiment with a $3.9 \mathrm{~cm}$ amplitude are given. On the left the image is shown where attenuation correction of the phased PET scan was applied with phased and RC-CT data. The image in the middle shows the same slice and phase of the same PET scan, but now attenuation correction was applied with the use of a non-RC CT scan. For both images of the phased PET (mid and left, Fig. 4) the same window width and window level settings were used, scaled between 0 to the maximum activity concentration found in the dataset with phased attenuation correction. The image on the right in Fig. 4 is the non-RC PET image of the same experiment. All three images show the same area. Because the amplitude $(3.9 \mathrm{~cm})$ is larger than the sphere diameter $(3.2 \mathrm{~cm})$ the non-RC PET image (right) shows a region with low activity in the middle. 


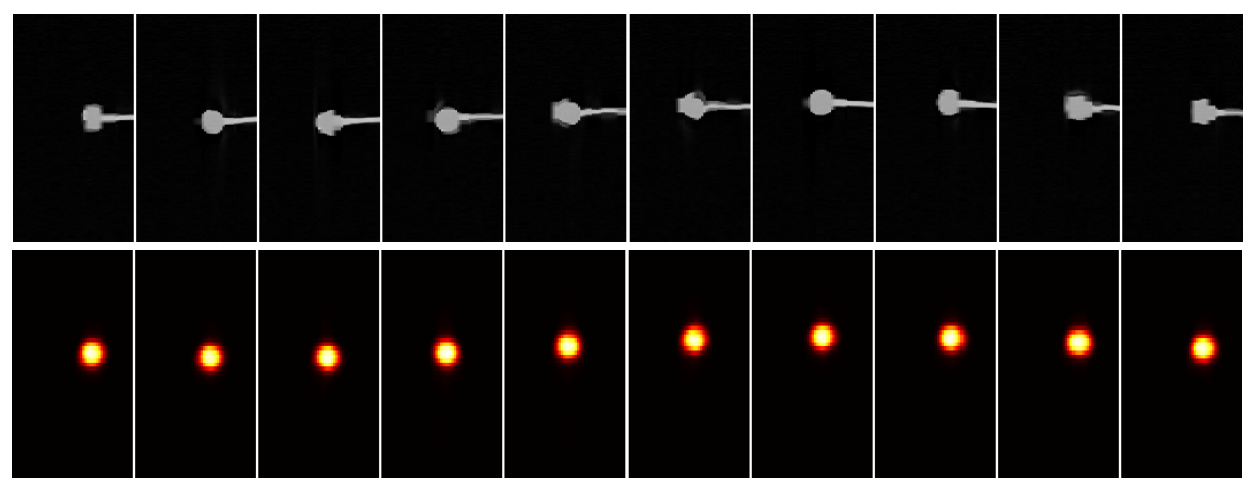

Fig. 3: 10 phases of the respiration correlated PET (bottom) and CT (top) data for a motion amplitude of $2.5 \mathrm{~cm}$.

Measurements of the maximum activities were performed for the ten phases of both the phased attenuation correction and the non-respiratory correlated attenuation correction.

The dataset obtained with non-correlated attenuation correction showed much larger standard deviations than the dataset with phased attenuation correction. Average maximum activity concentrations of $44864 \pm 6054 \mathrm{~Bq} / \mathrm{ml}(13.5 \%)$ and $40066 \pm 5142 \mathrm{~Bq} / \mathrm{ml}(12.8 \%)$ were found for the two experiments with noncorrelated attenuation correction, while in the data with phased attenuation correction average maximum activity concentrations were $49431 \pm 622 \mathrm{~Bq} / \mathrm{ml}$ $(1.3 \%)$ and $49852 \pm 771 \mathrm{~Bq} / \mathrm{ml}(1.5 \%)$.

In the experiment with a $2.5 \mathrm{~cm}$ amplitude the average and maximum difference between the two correction methods were $9.2 \%$ and $28.2 \%$ respectively. In the second experiment average and maximum deviations of respectively, $19.6 \%$ and $30.0 \%$ were found between the two correction methods (Table 1 ).
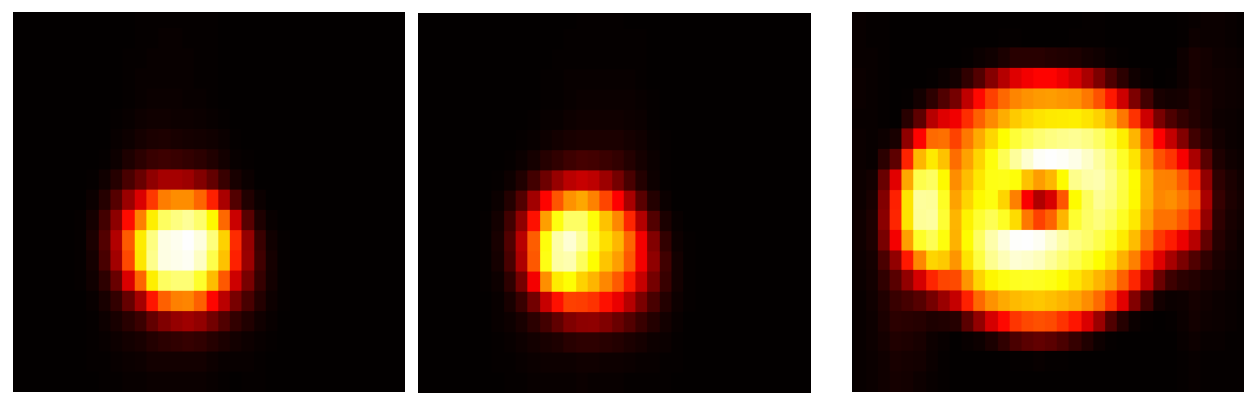

Figure 4: PET image with phased and respiration correlated attenuation correction (left), with non-RC attenuation correction (mid) and of the non-respiration correlated PET (right). The two phased images were made with the same window width and window level settings; other settings were used for the non-respiration correlated image. All three images show the same area. 
Table 1: Percentage difference, for the 10 phases, between the maximum activity concentrations measured in data reconstructed with non-respiration correlated attenuation correction and those measured in data that was reconstructed with phased attenuation correction.

\begin{tabular}{|ccc|}
\hline Phase & $\begin{array}{c}\text { Experiment } 1 \\
(2.5 \mathrm{~cm} \text { amplitude })\end{array}$ & $\begin{array}{c}\text { Experiment } 2 \\
(3.9 \mathrm{~cm} \text { amplitude })\end{array}$ \\
\hline 1 & 2.4 & -30.0 \\
2 & -0.3 & -28.7 \\
3 & -14.8 & -29.7 \\
4 & -28.2 & -4.6 \\
5 & -26.3 & -2.5 \\
6 & -20.5 & -21.0 \\
7 & -11.1 & -20.2 \\
8 & -0.2 & -14.5 \\
9 & 3.1 & -16.7 \\
\hline 10 & 4.2 & -28.4 \\
\hline average & -9.2 & -19.6 \\
\hline
\end{tabular}

The maximum value found in the non-RC PET data of experiment 1 with a 2.5 $\mathrm{cm}$ amplitude was $52839 \mathrm{~Bq} / \mathrm{ml}$, which is $7 \%$ higher than the average maximum activity concentration $(49431 \mathrm{~Bq} / \mathrm{ml})$ found for the RC-PET data with the same amplitude. In the experiment with a $3.9 \mathrm{~cm}$ amplitude a maximum activity concentration of $12316 \mathrm{~Bq} / \mathrm{ml}$ was found for the non-RC PET data, that is $75 \%$ lower than the average maximum activity concentration $(49852 \mathrm{~Bq} / \mathrm{ml})$ of the RC-PET data of the same experiment.

\section{Motion amplitude}

The center positions of the spheres for the different phases of CT and PET, reconstructed with respiration correlated attenuation correction, are plotted in Fig. 5. In Table 2 an overview of the movement amplitudes is given. 

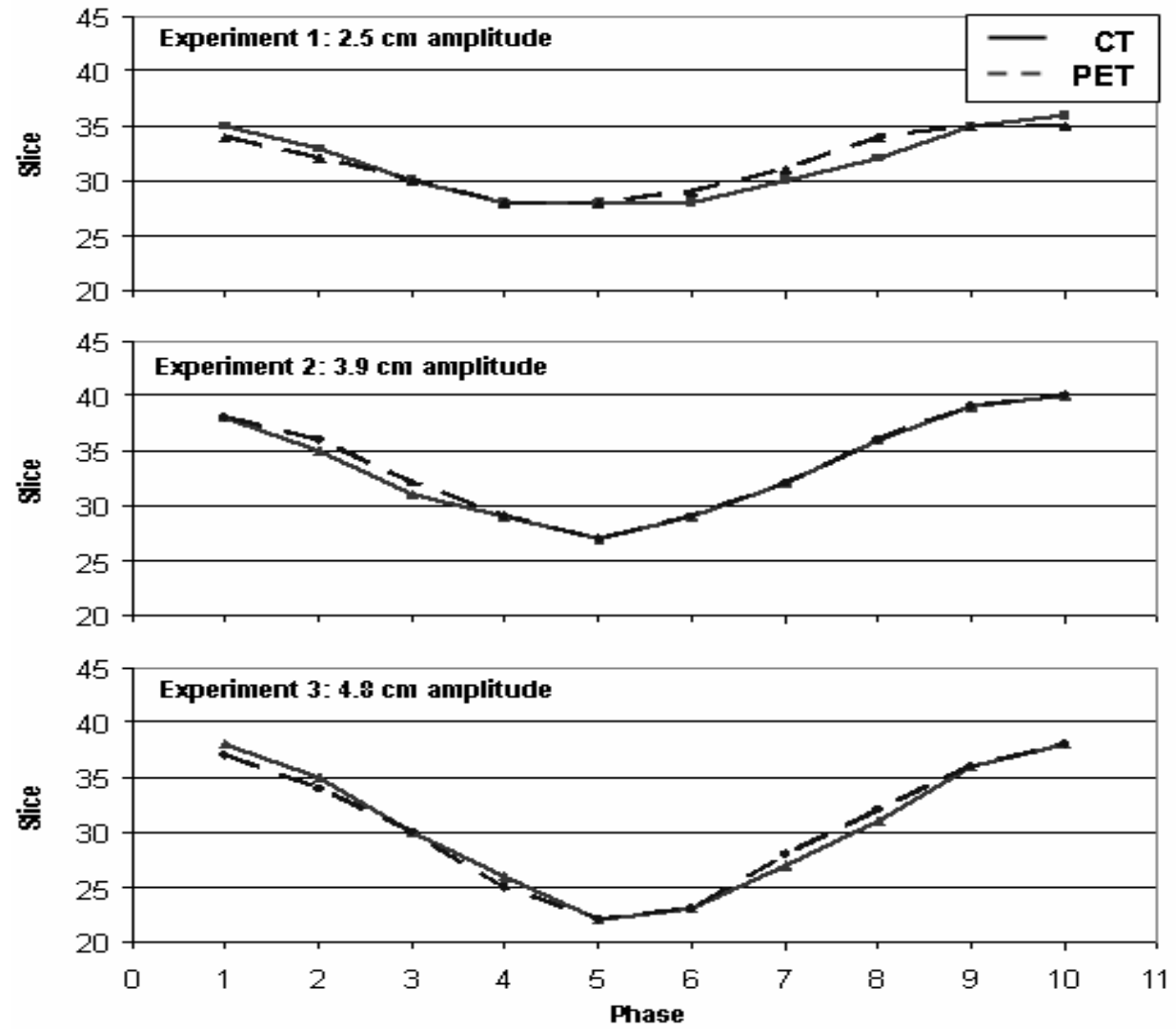

Figure 5: Centre position of the sphere at the ten phases on CT and PET, reconstructed with respiration correlated attenuation correction, images for three movement amplitudes of 2.5, 3.9 and $4.8 \mathrm{~cm}$, respectively.

Table 2: Movement amplitudes $(\mathrm{cm})$ measurement accuracy determined for the three experiments in both CT and PET, attenuation correction was performed with respiration correlated CT data, compared to the true amplitude.

\begin{tabular}{|cccc|}
\hline Experiment & True & CT & PET \\
\hline 1 & $2.5 \pm 0.1$ & $2.4 \pm 0.3$ & $2.1 \pm 0.3$ \\
2 & $3.9 \pm 0.1$ & $3.9 \pm 0.3$ & $3.9 \pm 0.3$ \\
3 & $4.9 \pm 0.1$ & $4.8 \pm 0.3$ & $4.8 \pm 0.3$ \\
\hline
\end{tabular}

\section{Volume}

In Table 3 an overview of the CT and PET volumes, for the clinical and phased data, is given. The actual sphere volume is $16.0 \mathrm{cc}$. 
In the non-RC CT scans the volume was underestimated by $22 \%$ and $46 \%$ in the two experiments. The PET volume in the non-RC scans on the other hand was overestimated by $156 \%$ in the first experiment and by as much as $370 \%$ in the second experiment.

The phased scans of CT show that the protocol used here results in measured volumes of $16.1 \pm 0.7,16.2 \pm 0.6$ and $16.7 \pm 0.9 \mathrm{cc}$ for the three experiments, respectively. The volumes found in the PET images are $16.2 \pm 0.6,16.5 \pm 0.3$ and $16.1 \pm 0.7 \mathrm{cc}$, respectively.

The average and maximum deviation from the true volume was $1.4 \%$ and $8.1 \%$ (experiment 3, phase 4) respectively for PET. The volumes determined with CT have an average deviation of $1.9 \%$. A maximum deviation of $10.6 \%$ (experiment 1 , phase 8 and experiment 3, phase 1) was found for CT based volumes.

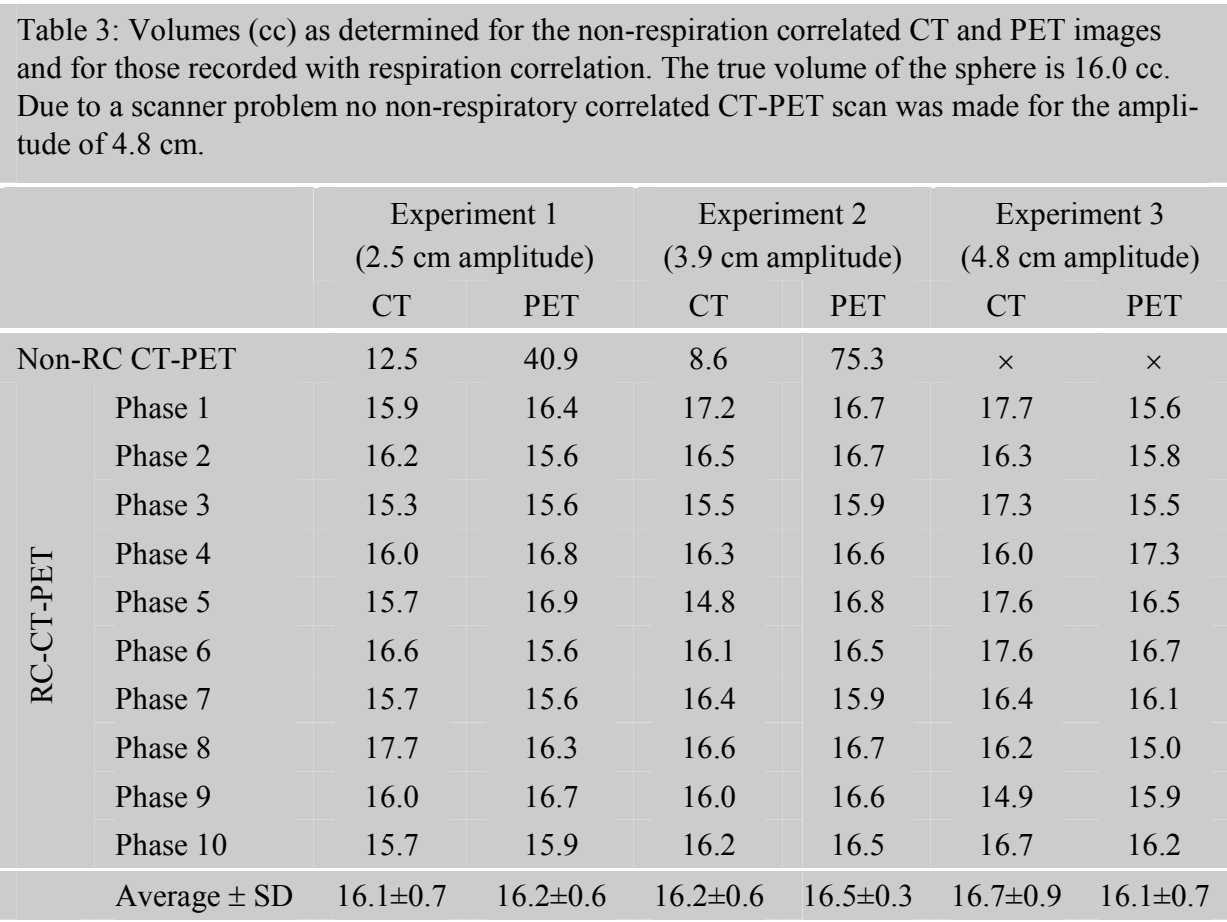

\section{DISCUSSION}

This phantom study showed that phased attenuation correction of respiration correlated PET images compared to non-respiration correlated attenuation correction leads to a more accurate localization of the tumor, an improved tumor volume definition, and a more precise determination of the activity concentra- 
tion, and more particularly the maximum activity concentration. Respiration correlated scanning and phased attenuation correction will therefore offer new and more precise information of the tumor, thus providing a better basis for radiation treatment planning. With information of tumor motion, internal margins can be determined for each patient individually and adapted to the actual movement in each direction. Moreover, with respiration correlation and phased attenuation correction an SUV determination is more reliable and thus better suited for use in tumor characterization and automatic delineation. To achieve good image quality in respiration correlated PET imaging in patients an optimal acquisition time and number of bins should be further investigated in a patient trial.

In Fig. 4 the image with non-respiration correlated attenuation correction shows that the maximum activity is clearly misplaced. In clinical practice such a motion artifact could result in a mislocalization of the tumor. Moreover the average maximum activity concentration found in the sphere is lower than in the data that was corrected with phased CTs. In a recent study with a gated, prospectively binned, PET protocol Nehmeh et al. ${ }^{15}$ compared attenuation correction with a non-RC CT to RC-CT. They found that phased attenuation correction led to an improved match of CT and PET data of up to $41 \%$, while maximum SUV was increased by a maximum of $16 \%$. Pevsner et al. ${ }^{8}$ found an underestimation of the activity concentration in the sphere between 19 and $75 \%$.

In the current study the difference in average maximum activity concentrations between the two attenuation correction methods reached up to $31 \%$, with an average of about $17 \%$ for the two experiments combined. It must be kept in mind however that in our experiments the spheres were simply placed in air, and little actual attenuation will have occurred. One could argue that the difference between phased and non-RC attenuation correction might therefore even be bigger in the presence of more attenuation. However, in the imaging of moving lung tumors, not all the attenuating tissues will move to the same degree as the tumor. The correction of attenuation that was caused by less mobile tissues will therefore not improve distinctly with the use of phased attenuation correction. The benefits of phased attenuation correction will largely depend on the relative contribution to the attenuation by moving tissues as compared to the more stationary tissues and might thus not be as striking as suggested by the results presented in this paper.

The volume of the sphere is greatly underestimated in non-RC CT images in this study, up to $46 \%$ deviation from the true volume. Others have found volume deviations between minus $35 \%$ and plus $79 \%$ in non-RC CT in comparison with $\mathrm{RC}-\mathrm{CT}{ }^{15,21}$. This large range, both underestimating and overestimating the 
volume, emphasizes again the importance of using corresponding RC-CT images for attenuation correction.

Volumes determined from the respiration correlated CT and PET images both show only minor deviations from the true volume, $1.9 \%$ and $1.4 \%$ on average for the respective modalities. This good result demonstrates the accuracy of both the phase binning process and the phased attenuation correction.

Various publications ${ }^{15,22-24}$ have reported a decrease in tumor volume when using gated PET as compared to non-RC scanning, regardless of their attenuation correction methods. The volumes determined in the current study from the non-RC data confirm that in PET smearing appears with a non-RC scanning protocol (Table 3). When the motion amplitude is smaller than the sphere's diameter, as was the case in experiment 1 with a $2.5 \mathrm{~cm}$ amplitude, nonrespiration correlated PET data showed a average maximum activity concentration that was $7 \%$ higher than the average maximum activity concentration found with the RC-PET data. This high average maximum activity concentration was found in the centre region of the motion, where some part of the sphere was present in every phase. In the $3.9 \mathrm{~cm}$ amplitude experiment the average maximum activity concentration found in the non-RC data was 75\% lower than found with RC-PET. Since the volume is determined with a threshold of $34 \%$ of the maximum activity concentration, the threshold value is set too low and will thus cause an even bigger overestimation of the PET volume than smearing alone. The difference in the ratio between the sphere diameter and the motion amplitude thus can explain the relatively bigger overestimation of the PET volume with the non-RC PET scan in experiment 2, which had a deviation of $370 \%$ from the actual volume as compared to a deviation of $156 \%$ in experiment 1 .

The motion amplitudes found with RC-PET and RC-CT (Table 2) corresponds well with the actual displacement. Taking the measurement error of $3 \mathrm{~mm}$, equal to the slice thickness, and the partial volume averaging effect into consideration the calculated differences will not be significant. This suggests an accurate phase binning for both modalities.

The CT and PET phases in experiments 2 and 3 (motion amplitudes of 3.9 and $4.8 \mathrm{~cm}$, respectively) show good correlation. The determinations of the sphere centre in experiment 1 , however, indicate that a slight phase shift was still present. Because the respiratory signals were recorded with different devices the starting point of the first phase bin in CT and PET was determined with different software. For both systems the maximum amplitude of the respiratory signal was set as the starting point. Differences in the respiration measurement techniques of the two systems may have caused the resultant phase shift. Synchronization of both modalities with a single device for respiration correlation will most likely dispose of this resulting phase difference. 


\section{CONCLUSION}

Valuable information is lost when scans, either CT or PET, are not correlated with respiration. Appropriate attenuation correction is at least as important. Phased attenuation correction not only gives a more accurate overall correction, but perhaps even more important, it provides a better match of PET and CT and results in a more reliable SUV and tumor volume. Combining RC-CT-PET scanning with phased attenuation correction will result in images with less smearing, less motion artifacts and thus in improved volume estimation and localization and quantification of the activity concentration.

In future studies the feasibility of phased attenuation correction in respiration correlated CT-PET for lung cancer patients will be examined.

\section{REFERENCES}

1. Ruysscher, D.D., et al., Effects of radiotherapy planning with a dedicated combined PETCT-simulator of patients with non-small cell lung cancer on dose limiting normal tissues and radiation dose-escalation: A planning study. Radiother Oncol, 2005. 77(1): p. 5-10

2. Senan, S., et al., Literature-based recommendations for treatment planning and execution in high-dose radiotherapy for lung cancer. Radiother Oncol, 2004. 71(2): p. 139-46.

3. Seppenwoolde, Y., et al., Precise and real-time measurement of 3D tumor motion in lung due to breathing and heartbeat, measured during radiotherapy. Int J Radiat Oncol Biol Phys, 2002. 53(4): p. 822-34.

4. Shimizu, S., Impact of respiratory movement on the CT images of small lung tumors in 3D radiotherapy. Int J Radiat Oncol Biol Phys, 2000. 46(5): p. 1127-33.

5. Goerres, G.W., et al., PET-CT image co-registration in the thorax: influence of respiration. Eur J Nucl Med Mol Imaging, 2002. 29(3): p. 351-60.

6. Beyer, T., et al., Dual-modality PET/CT imaging: the effect of respiratory motion on combined image quality in clinical oncology. Eur J Nucl Med Mol Imaging, 2003. 30(4): p. 58896.

7. Bockisch, A., et al., Positron emission tomography/computed tomography--imaging protocols, artifacts, and pitfalls. Mol Imaging Biol, 2004. 6(4): p. 188-99.

8. Pevsner, A., et al., Effect of motion on tracer activity determination in $\mathrm{CT}$ attenuation corrected PET images: A lung phantom study. Med Phys, 2005. 32(7): p. 2358-62.

9. Vansteenkiste, J.F., et al., Prognostic importance of the standardized uptake value on (18)Ffluoro-2-deoxy-glucose-positron emission tomography scan in non-small-cell lung cancer: An analysis of 125 cases. Leuven Lung Cancer Group. J Clin Oncol, 1999. 17(10): p. 32016.

10. Vedam, S.S., et al., Acquiring a four-dimensional computed tomography dataset using an external respiratory signal. Phys Med Biol, 2003. 48(1): p. 45-62.

11. Ford, E.C., et al., Respiration-correlated spiral CT: a method of measuring respiratoryinduced anatomic motion for radiation treatment planning. Med Phys, 2003. 30(1): p. 88-97.

12. Boucher, L., et al., Respiratory gating for 3-dimensional PET of the thorax: feasibility and initial results. $J$ Nucl Med, 2004. 45(2): p. 214-9. 
13. Wolthaus, J.W., et al., Fusion of respiration-correlated PET and CT scans: correlated lung tumour motion in anatomical and functional scans. Phys Med Biol, 2005. 50(7): p. 1569-83.

14. Nehmeh, S.A., et al., Quantitation of respiratory motion during 4D-PET/CT acquisition. Med Phys, 2004. 31(6): p. 1333-8.

15. Nehmeh, S.A., et al., Four-dimensional (4D) PET/CT imaging of the thorax. Med Phys, 2004. 31(12): p. 3179-86.

16. Goerres, G.W., et al., Respiration-induced attenuation artifact at PET/CT: technical considerations. Radiology, 2003. 226(3): p. 906-10.

17. Kinahan, P.E., B.H. Hasegawa, and T. Beyer, X-ray-based attenuation correction for positron emission tomography/computed tomography scanners. Semin Nucl Med, 2003. 33(3): p. 166-79.

18. Kinahan, P.E., et al., Attenuation correction for a combined 3D PET/CT scanner. Med Phys, 1998. 25(10): p. 2046-53.

19. Erdi, Y.E., et al., Segmentation of lung lesion volume by adaptive positron emission tomography image thresholding. Cancer, 1997. 80(12 Suppl): p. 2505-9.

20. Daisne, J.F., et al., Tri-dimensional automatic segmentation of PET volumes based on measured source-to-background ratios: influence of reconstruction algorithms. Radiother Oncol, 2003. 69(3): p. 247-50.

21. van Sornsen de Koste, J.R., et al., Tumor location cannot predict the mobility of lung tumors: a 3D analysis of data generated from multiple CT scans. Int J Radiat Oncol Biol Phys, 2003. 56(2): p. 348-54.

22. Nehmeh, S.A., et al., Effect of respiratory gating on quantifying PET images of lung cancer. J Nucl Med, 2002. 43(7): p. 876-81.

23. Nehmeh, S.A., et al., Effect of respiratory gating on reducing lung motion artifacts in PET imaging of lung cancer. Med Phys, 2002. 29(3): p. 366-71.

24. Erdi, Y.E., et al., The CT motion quantitation of lung lesions and its impact on PETmeasured SUVs. J Nucl Med, 2004. 45(8): p. 1287-92. 


\section{H A P T E R}

Respiratory-Gated CT as a Tool for the Simulation of Breathing Artifacts in PET and $\mathrm{PET} / \mathrm{CT}$

Med Phys. 2007; Accepted for publication

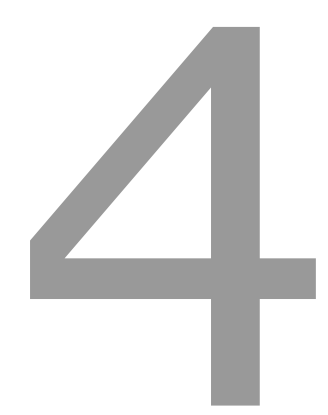




\begin{abstract}
Respiratory motion in PET and PET/CT studies blurs the images of tumors and causes attenuation-related errors. In rare instances, this problem even causes localization errors and the disappearance of tumors that should be detectable. Attenuation errors are severe near the diaphragm and can be enhanced when the attenuation correction is based on a CT series acquired during a breath-hold. To quantify the errors and identify the parameters associated with them, we performed a simulation based on respiratory-gated CT studies of five lung cancer patients. Diaphragmatic motion ranged from 8 to $25 \mathrm{~mm}$ in the five patients. The CT series were converted to $511-\mathrm{keV}$ attenuation maps which were forward-projected and exponentiated to form sinograms of PET attenuation factors at each phase of respiration. The CT images were also segmented to form a PET object, moving with the same motion as the CT series. In the moving PET object, spherical 20-mm mobile tumors were created in the vicinity of the dome of the liver, and immobile $20-\mathrm{mm}$ tumors in the mid chest region. The moving PET objects were forward-projected and attenuated, then reconstructed in several ways: phase-matched PET and CT; gated PET with ungated CT; ungated PET with gated CT; and conventional PET. Spatial resolution and statistical noise were not modeled. In each case, tumor uptake recovery factor was defined by comparing the maximum reconstructed pixel value with the known correct value. Phase-matched gated PET and CT gave essentially perfect PET reconstructions in the simulation. Gated PET with ungated CT gave tumors of the correct shape, but recovery was too large by an amount that depended on the extent of the motion, as much as $90 \%$ for mobile tumors and $60 \%$ for immobile tumors. Gated CT with ungated PET resulted in blurred tumors and caused the recovery errors between $-50 \%$ and $+75 \%$. Recovery in clinical scans would be $0 \%$ to $20 \%$ lower than stated, because spatial resolution was not included in the simulation. Mobile tumors near the dome of the liver were subject to the largest errors in either case. Conventional PET for $20 \mathrm{~mm}$ tumors was quantitative in cases of motion less than $15 \mathrm{~mm}$, because of canceling errors in blurring and attenuation, but the recovery factors were too low by as much as $30 \%$ in cases of motion greater than $15 \mathrm{~mm}$. Quantitative PET imaging near the diaphragm requires proper matching of attenuation information to the emission information. The problem of missed tumors near the diaphragm can be reduced by acquiring attenuation correction information near end expiration. A simple $\mathrm{PET} / \mathrm{CT}$ protocol requiring no gating equipment also addresses this problem.
\end{abstract}




\section{INTRODUCTION}

Accurate measurement of tumor standard uptake values (SUV) is an important requirement in many PET applications. In whole-body PET studies SUV is believed to be an indicator of tumor avidity and a predictor of patient survival rates $^{1-4}$, and accurate quantitation is important when autodelineation is used in therapy planning ${ }^{5-8}$. Indeed, an important requirement for PET in oncology is to detect every tumor that is large enough and has enough tracer uptake to be detectable, even when measurement errors have the potential to cause imagereconstruction errors that result in a missed or mislocated tumor. Respiratory motion is one factor with the potential to reduce quantitative accuracy and to cause missed or misplaced tumors near the diaphragm. For example, the report by Keall et al. shows that significant motion is observed in many regions of significance to oncology ${ }^{9}$ and the report by Osman et al. indicates the possibility of localization and detection errors near the diaphragm ${ }^{10}$. In the state-of-the-art, however, PET and PET/CT reconstruction algorithms are based on the assumption that the patient does not move during the measurement of the emission sinogram and the attenuation factors (AF).

Motion causes two phenomena which lead to image artifacts. First, the tracer distribution moves, blurring the image. Second, the AFs change with time, leading to too much or too little correction for attenuation on some lines of response. The latter problem is severe near the diaphragm and is sometimes enhanced when the AFs are based on a CT series acquired during a breath-hold, with the associated problem that lesions in fusion images of CT and PET, could be mislocated, e.g. from the liver into the lung ${ }^{10,11}$. One goal of this paper is to indicate clearly, in the case of PET tumor imaging, what kinds of error arise from respiratory motion in conventional PET (CP), considering the complicated interplay between these two sources of error.

Another goal of this paper is to demonstrate the advantages of respiratory gating $(\mathrm{RG})$ in acquisition of the PET or AF information ${ }^{12-14}$. We are interested in respiratory-gated PET (GP), in respiratory-gated CT (GCT), in PET/CT performed with attenuation measurements from non-gated $\mathrm{CT}$, in non-gated PET/CT with gated CT, and in PET/CT in which both modalities are gated. Practical problems are associated with clinical and phantom-based investigations of the benefits of gating. One problem is the low statistics in each phase of GP. Another problem is that laboratory studies are likely to use an unrealistic model of the breathing seen in patients. In this paper, we avoid those problems by using GCT as the basis for a simulated PET emission data set, created with a digital computer. To our knowledge such a simulation has not yet been reported in the literature. A preliminary version of this work was presented earlier ${ }^{15}$. 
This approach can address the following issues. How well does conventional PET do in the presence of such motion? Does the answer depend strongly on the location of the tumor that is affected by motion? Is phase matching of GP and GCT quantitative? Does GP or GCT by itself solve the motion problem, delivering a quantitative result? What PET/CT imaging protocols, based on technology available today, address respiration-related errors?

\section{METHODS}

Our investigation is derived from a set of five GCT studies of lung cancer patients, selected at random from the population of patients receiving GCT at Maastro Clinic. Patients' breathing was monitored by a strain gauge worn around the abdomen ${ }^{16}$. Low-pitch helical CT scans of the chest were performed with a sixteen-slice CT scanner (Sensation 16, Siemens AG, Forchheim, Germany). The data for the five patients were retrospectively reconstructed into images representing a $500 \mathrm{~mm}$ field of view sampled by a $512 \times 512$ matrix, with $3 \mathrm{~mm}$ between slices, and 10 phases of the respiration cycle starting at end inspiration. GCT images were converted to a map $\mu(\vec{b}, r)$ of PET attenuation coefficients ${ }^{17}$ at each phase of respiration $r$ and image location $\vec{b}$. These were transformed to a sinogram of AF values at those phases by applying a highresolution forward projector ${ }^{18}$ then rebinned to 168 radial and 168 angular bins. Our equation for this is

$$
A F(\vec{d}, r)=\exp (-R\{\mu(\vec{b}, r)\})
$$

where $\mathrm{R}$ is the Radon transform and $\vec{d}$ represents a sinogram bin. Related to this quantity is the average attenuation, which, if the patient's breathing pattern were perfectly regular, would be the same as the AF in a high-quality scan in a PET attenuation scan with transmission sources. For us the defining equation in the case of ten gates is

$$
A F(\vec{d})=\frac{1}{10} \sum_{r} A F(\vec{d}, r) .
$$

Other related quantities are the sinogram of attenuation correction factors (ACF) in each gate,

$$
A C F(\vec{d}, r)=\frac{1}{A F(\vec{d}, r)},
$$


and the ACF corresponding to the average attenuation,

$$
A C F(\vec{d})=\frac{1}{A F(\vec{d})},
$$

The CT series were also segmented to form a simulated PET volume in which a solitary tumor could be imbedded. The PET volumes were defined through a segmentation procedure whose parameters were determined by studying typical whole-body PET/CT studies. First, to enable a smooth segmentation, the volume was smoothed in three dimensions with a gaussian kernel whose full width at half maximum was $3 \mathrm{~mm}$. Next, the segmentation procedure was run. Pixels with CT numbers between -25 and $200 \mathrm{HU}$, representing soft tissue and marrow, were assigned a SUV of 1.0. Pixels between -300 and $-25 \mathrm{HU}$ (fat) were assigned SUV $=0.3$. Pixels between -950 and -300 (lung) were assigned SUV = 0.17 . Pixels above $200 \mathrm{HU}$ (bone) were assigned SUV $=0.1$. This procedure had several unrealistic characteristics. For example, the blood in the ventricles of the heart was treated like soft tissue and clothing and blankets were sometimes treated like fat.

By inspecting coronal and sagittal sections through the sequence of image volumes of each of the five patients, we located the dome of the liver (DOL) in each phase of respiration. We used a model in which the motion of the DOL is entirely in the superior / inferior (SI) direction, the most common direction of tumor motion in the survey of Keall et al. ${ }^{9}$ and in the study by Liu et al. ${ }^{19} \mathrm{We}$ simulated a solitary tumor in one of six places: three mobile tumors were simulated on the right side, and three immobile ones in the mid chest. The mobile ones were assigned a position relative to the DOL position in each gate. Each simulated tumor, mobile or immobile, was assigned $\mathrm{SUV}=5.0$ and a diameter of 10,20 , or $30 \mathrm{~mm}$. A mobile tumor denoted DOL was centered $5 \mathrm{~mm}$ below the DOL position. A mobile tumor denoted LRL was defined $25 \mathrm{~mm}$ higher than the DOL tumor, in the lower right lung, that is, $20 \mathrm{~mm}$ above the dome of the liver. A mobile tumor denoted ULL was defined $15 \mathrm{~mm}$ below the DOL tumor, in the upper lobe of the liver. The three immobile tumors were placed in the mid chest and were denoted MC1, MC2, and MC3. Their SI coordinates were set equal to the mean positions of the mobile LRL, DOL, and ULL tumors, respectively. The six tumor locations are indicated by Fig. 1. 


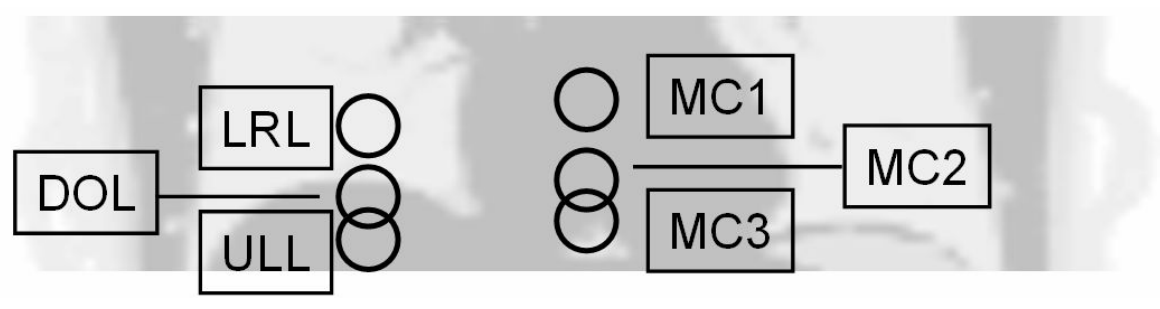

Figure 1. Coronal section through a segmented PET object, showing the locations of simulated tumors. The patient is shown at end inspiration. Abbreviations refer to gated lower right lung, dome of the liver, upper lobe of the liver, and mid-chest positions 1, 2, 3 .

Because there were in principle three tumor diameters, six locations, five patients, and ten phases of respiration, this procedure should lead to the creation of 900 simulated PET volumes to be processed in our study. However, we processed data for the 10 and $30 \mathrm{~mm}$ tumors in only a few cases when it became clear that most of the results could be understood by considering just the $20 \mathrm{~mm}$ tumors. The voxelized PET volume in each gate $\mathrm{r}$ and voxel $\vec{b}$ is denoted $O(\vec{b}, r)$. We transformed these into unattenuated 2D sinograms, $p_{\text {no_att }}(\vec{d}, r)$, using the forward projector referred to above, again with 168 radial and 168 angular samples. The equation for this is

$$
p_{\text {no_att }}(\vec{d}, r)=R\{O(\vec{b}, r)\} \text {. }
$$

Attenuated 2D PET sinograms were made by multiplying the PET sinograms by the AF sinograms:

$$
p(\vec{d}, r)=A F(\vec{d}, r) \times p_{\text {no_att }}(\vec{d}, r) .
$$

These would be the sinograms measured by a PET scanner with respiratory gating, apart from effects such as limited spatial resolution, scattered radiation, and spatial sensitivity variations. Those effects were not included in the simulation, so that the study could focus exclusively on effects due to motion. We also were interested in the time-averaged sinogram, defined as

$$
p(\vec{d})=\frac{1}{10} \sum_{r} p(\vec{d}, r)
$$

Six types of PET volume were extracted for analysis and comparison. The first of these was $O(\vec{b}, r)$, described above. Second was the time average of these, denoted 
$O(\vec{b})=\frac{1}{10} \sum_{r} O(\vec{b}, r)$

Third was the series derived by reconstructing the gated sinograms with attenuation correction derived from the matched CT gate. These were made with filtered backprojection according to the equation

$$
I_{G P-G C T}(\vec{b}, r)=R^{-1}\{p(\vec{d}, r) \times A C F(\vec{d}, r)\},
$$

where $R^{-1}$ is the inverse Radon transformation. Although iterative reconstruction is now the usual reconstruction method in whole-body PET ${ }^{20,21}$, filtered backprojection is appropriate in the absence of statistical noise, because it is a gold standard and because there is no question of when to stop the iterations. With the exceptions defined by equations 13 and 14 below, we do not blur the reconstructed images to give a clinical level of spatial resolution.

The fourth PET series to be compared was derived by reconstructing gated sinograms with the average attenuation. This series was associated with the respiratory gate index used for PET reconstruction. It represents, under the assumption of regular breathing, what is achievable when gated PET is used on a PET scanner with a conventional measurement of the attenuation, i.e. a 511-keV transmission scan with rotating rods of ${ }^{68} \mathrm{Ge}$. The defining equation is

$$
I_{G P}(\vec{b}, r)=R^{-1}\{p(\vec{d}, r) \times A C F(\vec{d})\} .
$$

Similarly, the fifth PET series to be compared was obtained when the emission sinograms were time-averaged, i.e. not gated, but the CT was gated. The PET series is associated with the respiratory gate index of the CT that is used for AC. Considering this dataset, one can ask whether a particular state of respiration can be used for attenuation correction. Also, we can investigate what happens when the free-breathing patient is imaged with a very fast CT scan at an arbitrary state of respiration. The defining equation for this case is

$$
I_{G C T}(\vec{b}, r)=R^{-1}\{p(\vec{d}) \times A C F(\vec{d}, r)\} .
$$

Conventional PET was the sixth PET series to be compared. In this case, both the emission and attenuation were time-averaged. Again operating under the assumption that one breathing cycle is the same as a long time average, we have the following defining equation.

$$
I_{C P}(\vec{b})=R^{-1}\{p(\vec{d}) \times A C F(\vec{d})\}=\frac{1}{10} \sum_{r} I_{G P}(\vec{b}, r) .
$$


The seventh, final type of PET image to be compared was the GCT image based on $\mathrm{CT}$ acquired at EE or EI. In these cases, and these cases only, we attempted to model the spatial resolution of state-of-the-art PET scanners. Our equations for these images are

$$
I_{G C T-E E}(\vec{b})=\left.I_{G C T}(\vec{b}, r)\right|_{r=E E} \otimes g(\vec{b})
$$

and

$$
I_{G C T-E I}(\vec{b})=\left.I_{G C T}(\vec{b}, r)\right|_{r=E I} \otimes g(\vec{b}),
$$

where the symbol $\otimes$ represents convolution and $\mathrm{g}$ is a three-dimensional gaussian kernel, whose full width at half maximum was set to $6.4 \mathrm{~mm}$ to represent 4 $\mathrm{mm}$ instrument resolution and $5 \mathrm{~mm}$ smoothing to reduce image noise.

We analyzed each of these PET volumes in three ways, based on a maximumintensity coronal projection over a $10-\mathrm{mm}$ coronal slice that contained the tumor. First, the SUV of the tumor was defined as the maximum pixel value in the coronal slice containing the tumor. We converted this to a percent recovery figure by dividing by the expected SUV value (the value is 5.0) and multiplying by 100. This maximum-pixel method, and related methods based on regions around the maximum, are used commonly in clinical PET and PET/CT ${ }^{1-4}$. Second, the image of the tumor itself was inspected to see if the correct shape is obtained in the reconstruction. A round shape of the same diameter as the lesion $(10,20$, or $30 \mathrm{~mm}$ ) was expected. Third, the image of the surrounding tissues was inspected visually for artifacts.

\section{RESULTS}

\section{The diaphragmatic motion model}

The motion of the DOL in our model is characterized in each patient and at each phase of respiration by a single value, the $\mathrm{z}$ coordinate used to simulate the positions of the mobile tumors. The total excursions in $\mathrm{z}$ (and the root mean square) for the five patients, ranked from least motion to most motion, were: patient $1,8.4 \mathrm{~mm}(2.8 \mathrm{~mm})$; patient 2, $11.1 \mathrm{~mm}(3.4 \mathrm{~mm})$; patient 3, $13.8 \mathrm{~mm}$ $(4.3 \mathrm{~mm})$; patient 4, $19.8 \mathrm{~mm}(5.4 \mathrm{~mm})$; and patient 5, $25.2 \mathrm{~mm}(8.5) \mathrm{mm}$.

Patients 3 and 5 had actual large tumors in the lower right lung. In both cases, the diaphragm could be visualized in sagittal and coronal sections, allowing us to define the DOL unambiguously. In both cases, our methodology placed the 
simulated LRL tumor inside these actual masses. The other three patient studies had no such large masses in the lower right lung.

\section{Typical images}

Fig. 2 presents coronal sections through $O(\vec{b}, r), O(\vec{b}), \quad I_{G P-G C T}(\vec{b}, r)$, $I_{G P}(\vec{b}, r), I_{G C T}(\vec{b}, r)$, and $I_{C P}(\vec{b})$ for patient 4 . The figure also indicates which phases represent end expiration (EE) and end inspiration (EI) as determined from the DOL position. The percent recovery of the lesion SUV in each position is printed in each frame of the picture.

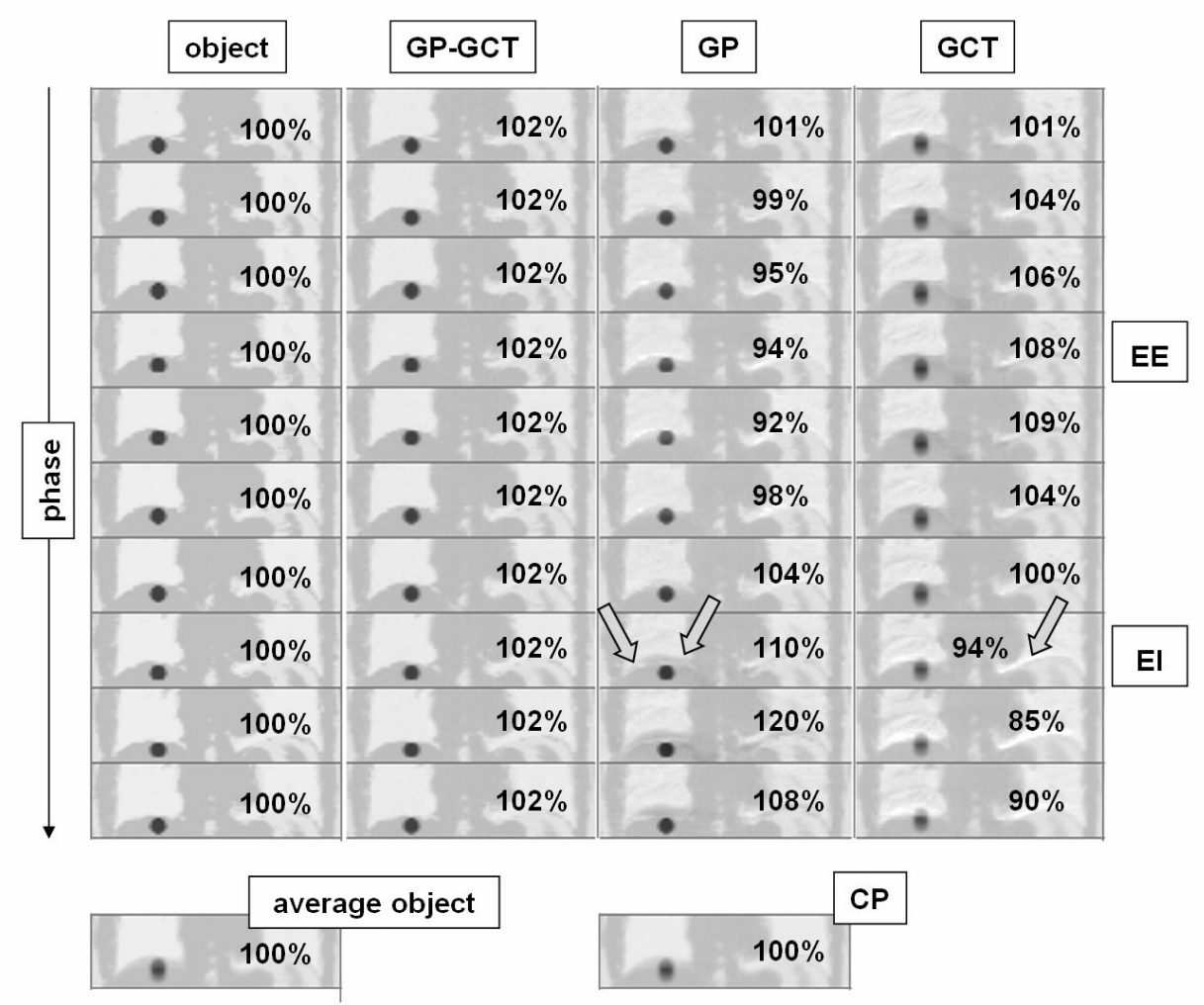

Figure 2. Coronal sections through PET series associated with the 20-mm dome of the liver tumor in patient 4 . The figure presents the PET object at each gate, the average object, three gated reconstructed image sequences, and a non-gated reconstruction, as explained in the text. SUV recovery is indicated with a percent value in each frame of the picture. Arrows indicate prominent artifacts. Abbreviations refer to gated PET, gated CT, conventional PET, end expiration, and end inspiration. 
In this simulation, the phase-matched reconstructions (GP-GCT) produced correct SUV recovery and created no striking artifacts. Actually, recovered values were in most cases $1 \%$ to $2 \%$ too high because of reconstruction noise.

Fig. 2 also indicates that the recovery varied from phase to phase when one used the GP and GCT methods. This is discussed in the following sections. Reconstruction artifacts were evident when one used either of those methods. They were particularly noticeable near the diaphragm. These are indicated with arrows in Fig. 2.

Using this approach, we simulated mobile lung lesions $20 \mathrm{~mm}$ in diameter in all six of the locations introduced above, and saved the results. We simulated 10 and $30 \mathrm{~mm}$ lesions in our earlier analysis ${ }^{15}$ of patient 5 and repeated the analysis of the 10 and $30 \mathrm{~mm}$ sizes for patient 4 . Our study of those two lesion diameters showed that the pattern of artifacts was the same as it was for the larger tumors, except that the $10 \mathrm{~mm}$ tumor's SUV recovery was worse than in the case of a $20 \mathrm{~mm}$ tumor, presumably because the motion is larger relative to its size, and that, conversely, the $30 \mathrm{~mm}$ tumor's SUV recovery was better. Believing that this was a trend, we focused our analysis on tumors with a simulated diameter of $20 \mathrm{~mm}$.

\section{Motion blurring and SUV recovery}

The motion-blurring phenomenon is best studied by examining the timeaveraged simulated tumor, $O(\vec{b})$. One would expect that respiration should blur edges in the object, and this was borne out by our study of the diaphragm image in $O(\vec{b})$, for example the bottom left corner of Fig. 2. The tumor's image was distended and blurred in the SI direction. Also, the diaphragm's sharp edges were replaced with diffuse ones. In $O(\vec{b})$, the tumor uptake determined as the maximum pixel was $100 \%$ in the mobile tumors in patients $1,2,3,4$ where the motion was less than the tumor diameter, but uptake was between $69 \%$ and $73 \%$ in patient 5 , where the motion exceeded the tumor diameter.

Fig. 3 illustrates the blurring of the moving tumors. The figure shows the 20$\mathrm{mm}$ tumors at the dome of the liver in patient 2, who had $11.3 \mathrm{~mm}$ of respiratory motion, and patient 5 , who had $25.2 \mathrm{~mm}$ of motion. The figure compares $\mathrm{EE}$ and EI, and includes a comparison with the time-averaged object and the conventional PET reconstruction (CP). One will notice a slight flattening at the edges of the sphere, due to our use of a model in which the separation between slices was $3 \mathrm{~mm}$. 


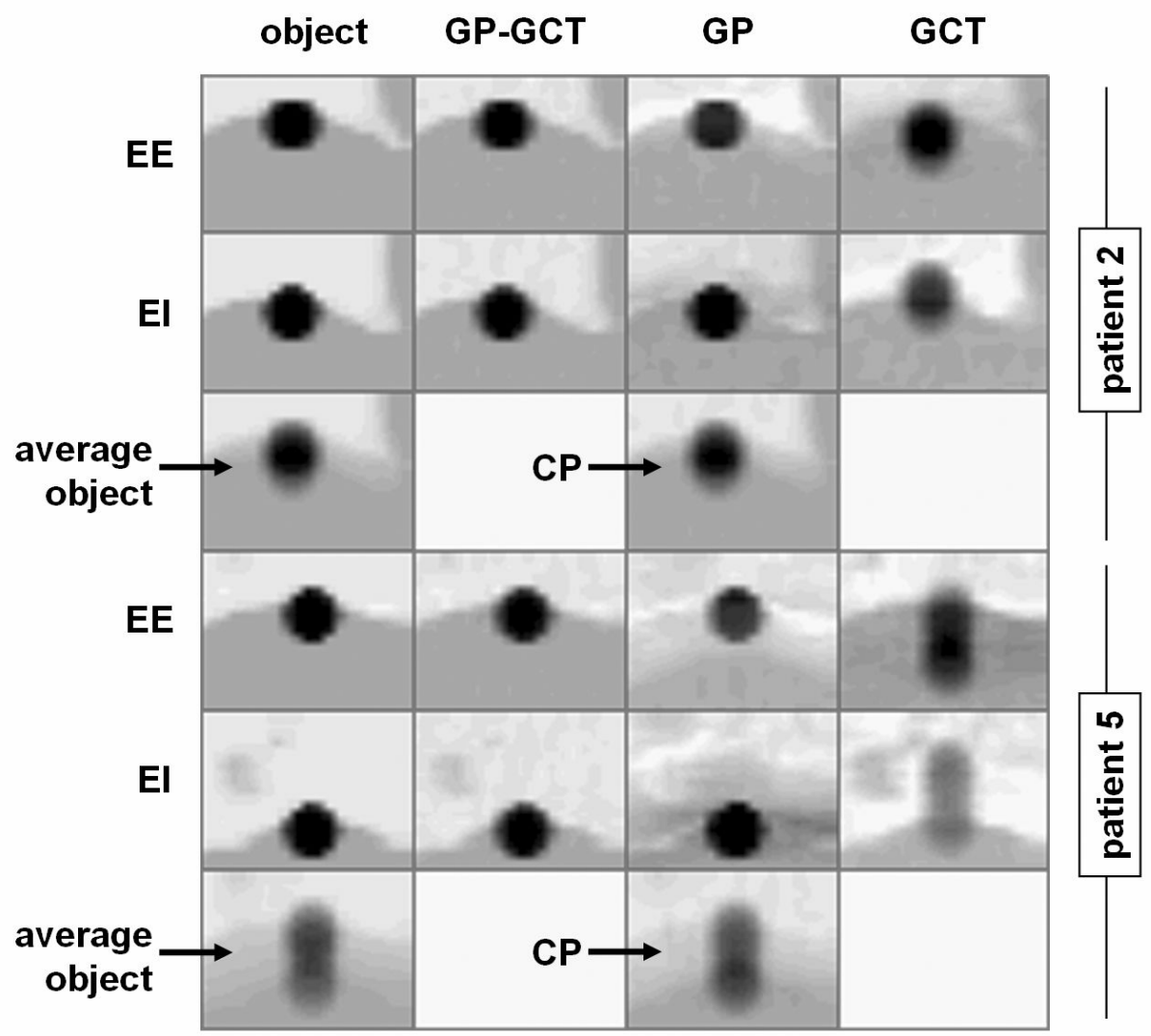

Figure 3. Coronal section through a simulated 20 -mm tumor in patients 2 and 5 at the dome of the liver, at respiratory phases EE and EI. Averages are also shown. Abbreviations refer to gated PET, gated CT, conventional PET, end expiration, and end inspiration.

The figure shows that phase-matched PET/CT, represented by the GP-GCT images, was in principle capable of generating an essentially perfect replica of the object itself at each phase of respiration. The tumor had the correct shape and was uniformly filled with the correct values. The surrounding tissues were also reproduced well. When the PET was gated but the CT was not, the shape of the tumor was correct but the distribution of activity inside the tumor was nonuniform, and SUV values in the surrounding tissues were wrong. When the CT was gated and the PET was not, the tumor was distorted and the background values were in error. Conventional PET, in these examples, gave nearly the same image as motion blurring alone.

Considering all six tumor locations in patients 1, 2, 3, who had less than $15 \mathrm{~mm}$ of breathing motion, and all ten phases of respiration, we found the range of values shown in Table 1. GP-GCT always gave essentially $100 \%$ recovery. In 
the case of GP, the largest error (overestimation of the recovery) occurred at or near EI and the most negative error (underestimation) occurred at or near EE.

Table 1. Range of SUV recovery for $20 \mathrm{~mm}$ lesions in patients 1, 2, and 3 who had less than 15 $\mathrm{mm}$ of breathing motion. The average value is presented if the range is less than $5 \%$. The values reported in the table are the smallest and largest recovery values in these three patients, considering all phases of respiration. Abbreviations refer to gated PET, gated CT, conventional PET, the lower right lung, the dome of the liver, the upper lobe of the liver, and three positions in the mid chest.

\begin{tabular}{|c|c|c|c|c|c|}
\hline \multirow{4}{*}{$\frac{\stackrel{0}{0}}{0}$} & & GP-GCT & GP & GCT & $\mathrm{CP}$ \\
\hline & LRL & $102 \%$ & $100-110 \%$ & $97-109 \%$ & $101 \%$ \\
\hline & DOL & $101 \%$ & $93-145 \%$ & $90-122 \%$ & $100 \%$ \\
\hline & ULL & $102 \%$ & $94-114 \%$ & $94-112 \%$ & $100 \%$ \\
\hline \multirow{3}{*}{$\begin{array}{l}\frac{0}{\tilde{0}} \\
\stackrel{\circ}{\Xi} \\
. \Xi\end{array}$} & MC1 & $102 \%$ & $99-108 \%$ & $97-119 \%$ & $102 \%$ \\
\hline & MC2 & $102 \%$ & $97-111 \%$ & $99-112 \%$ & $101 \%$ \\
\hline & MC3 & $102 \%$ & $92-115 \%$ & $93-120 \%$ & $101 \%$ \\
\hline
\end{tabular}

We also present the recovery values for patients 4 and 5, who had more than 15 $\mathrm{mm}$ of motion. These are summarized in Table 2.

Table 2. Range of SUV recovery for $20 \mathrm{~mm}$ lesions in patients 4 and 5 who had more than 15 $\mathrm{mm}$ of breathing motion. The average value is presented if the range is less than $5 \%$. The values reported in the table are the smallest and largest recovery values in these two patients, considering all phases of respiration. Abbreviations refer to gated PET, gated CT, conventional PET, the lower right lung, the dome of the liver, the upper lobe of the liver, and three positions in the mid chest.

\begin{tabular}{|c|c|c|c|c|c|}
\hline \multirow{4}{*}{$\begin{array}{l}\frac{0}{\pi 0} \\
\frac{0}{0} \\
\Xi\end{array}$} & & GP-GCT & GP & GCT & $\mathrm{CP}$ \\
\hline & LRL & $102 \%$ & $100-158 \%$ & $68-106 \%$ & $70-89 \%$ \\
\hline & DOL & $101 \%$ & $92-191 \%$ & $45-120 \%$ & $76-100 \%$ \\
\hline & ULL & $101 \%$ & $73-134 \%$ & $55-108 \%$ & $72-101 \%$ \\
\hline \multirow{3}{*}{$\begin{array}{l}\frac{0}{0} \\
0 \\
\text { : } \\
. \Xi\end{array}$} & $\mathrm{MC1}$ & $102 \%$ & $100-108 \%$ & $100-108 \%$ & $101 \%$ \\
\hline & $\mathrm{MC} 2$ & $102 \%$ & $85-164 \%$ & $91-130 \%$ & $102 \%$ \\
\hline & MC3 & $102 \%$ & $85-189 \%$ & $78-175 \%$ & $102 \%$ \\
\hline
\end{tabular}

We calculated the SUV recovery for all six tumors reconstructed by the GCT method at EE and EI, with gaussian filtering included, according to Equations 13 and 14. The recovery values are plotted in Fig. 4 as a function of the amount of diaphragmatic motion. 

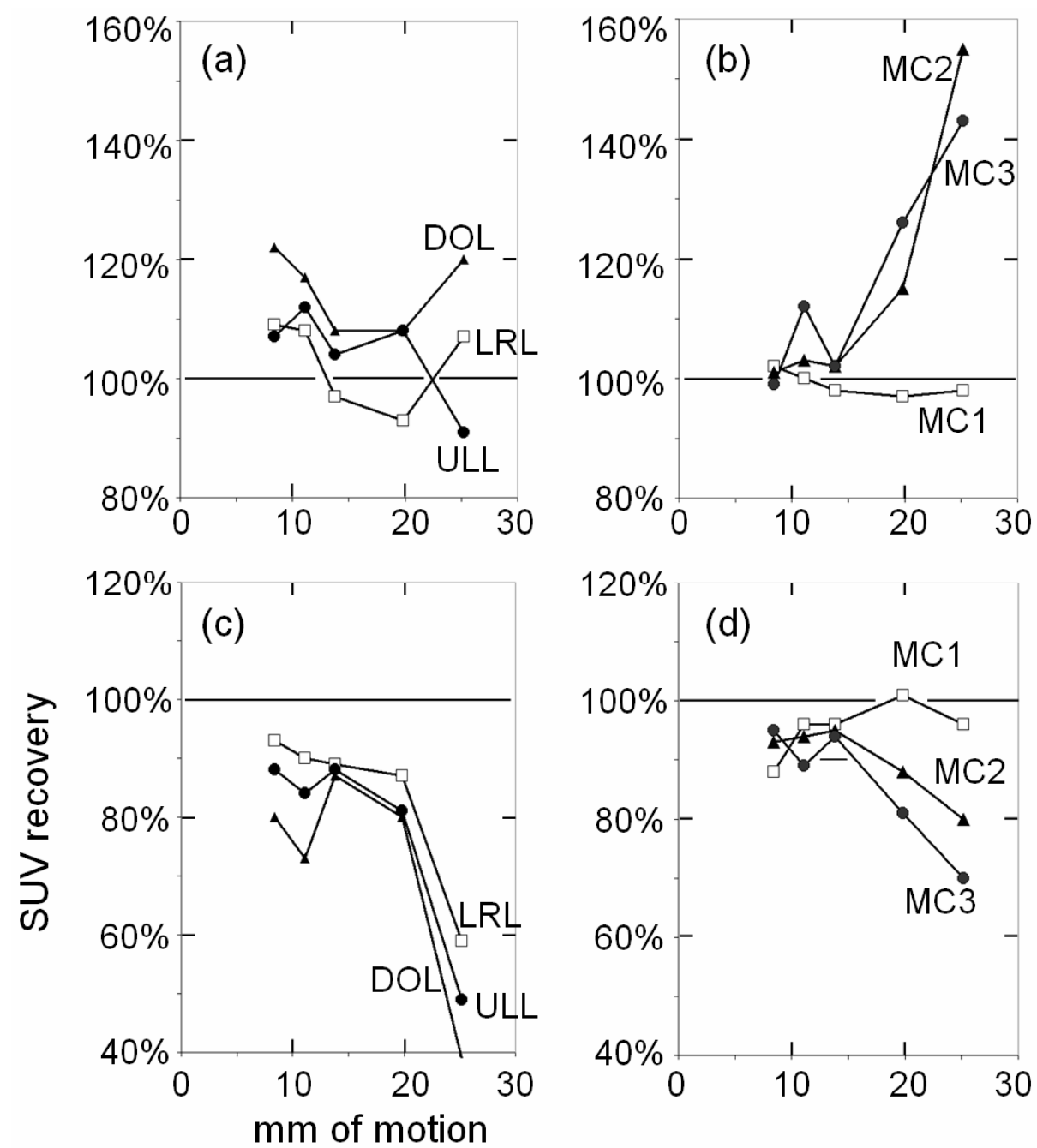

Figure 4. SUV recovery for $20 \mathrm{~mm}$ tumors measured with non-gated PET, reconstructed with (a,b) CT at end expiration and (c,d) CT at end inspiration. Six tumor locations are reported: (a,c) lower right lung, dome of the liver, and upper lobe of the liver; and (b,d) positions $1,2,3$ in the mid chest.

\section{DISCUSSION}

A quantitative PET or PET/CT reconstruction procedure requires compensation for the physical effects that distinguish the actual measurement from an ideal measurement based on unattenuated line integrals of the radioactivity density. Those effects include photon attenuation, statistical fluctuations, a background 
of scattered radiation, random coincidences, positron range effects, noncolinearity in the two annihilation photons, detector sensitivity variations, and patient motion. In oncological PET, attenuation is normally the largest of these effects, with attenuation factors sometimes in excess of 100 in abdominal and shoulder sections but about an order of magnitude less than that in lung sections. At the level of the diaphragm, this largest effect changes from moment to moment with the patient's breathing. Motion of the radioactive tracer in internal organs is also an important effect in this region in the breathing patient. At the outset of this investigation, it seemed to us that an understanding of the effect of breathing upon PET's accuracy in this anatomical region should include a realistic model of the diaphragm's actual motion in cancer patients. Respiratorygated CT made this possible, providing measurement of the anatomy of the entire chest.

Our study of respiratory motion effects in PET/CT was driven by GCT series from five patient studies. The range of diaphragmatic motion in this set of five studies was from 8 to $25 \mathrm{~mm}$, with three of the five cases showing motion less than $15 \mathrm{~mm}$. This range of motion is typical of the range seen in a number of clinics around the world ${ }^{9,19}$. We performed some calculations with 10 and 30 $\mathrm{mm}$ tumors but only presented results associated with $20 \mathrm{~mm}$ tumors. Large tumors, for example, $30 \mathrm{~mm}$, are less blurred by motion. Although breathing artifacts in the imaging of smaller tumors, for example $10 \mathrm{~mm}$, are of great concern clinically, in actual measurements the limitations of detector resolution, statistical noise, and partial volume effects preclude an accurate measurement of the uptake of such small tumors. Since good SUV recovery is possible for 20 $\mathrm{mm}$ lesions, we felt it was appropriate to simplify our presentation by considering mainly this tumor size. The actual resolution in a clinical scan depends on reconstruction parameters, for example the reconstruction method, the number of iterations in the case of iterative reconstruction, and the filtering parameter which is chosen to control statistical noise.

In our simulation, the combination of gated PET with gated CT provided accurate results for each patient and each tumor location at every phase of respiration. This indicates that the Radon transform of the PET object, generated by our forward projector, was correctly inverted by our filtered backprojection algorithm. This is a reassuring check on the correctness of the computational tools that we used.

Gating of the PET alone gave the correct tumor shape but was not quantitative. Gating of the CT alone gave a shape like the shape caused by motion blur alone, and was not quantitative. This was also true of conventional PET. A PET/CT study performed using a CT scan of a free-breathing patient should be similar to gated CT with a random choice of the respiratory phase, provided that the $\mathrm{CT}$ scan is performed rapidly enough, i.e. it unavoidably has the same kinds of errors as ungated PET with GCT. A PET/CT study with a CT scan at deep inspi- 
ration cannot be modeled by our simulation, since the position of the diaphragm is likely to be outside the range seen in the PET scan of a freely breathing patient.

Mobile tumors were more susceptible to quantitation errors because they were blurred and also subject to attenuation errors. Immobile tumors were subject only to attenuation errors, yet even these tumors cannot be assigned the correct values by GP or GCT alone. In our simulation, $\mathrm{CP}$ was more accurate than either GP or GCT in the imaging of $20 \mathrm{~mm}$ tumors. This appears to be due to canceling errors in emission blurring (underestimation) and attenuation correction (overestimation). Our limited study of 10 and $30 \mathrm{~mm}$ tumors indicated that motion blurring of $10 \mathrm{~mm}$ tumors was so great that the attenuation errors no longer compensated for it, whereas recovery for $30 \mathrm{~mm}$ tumors in $\mathrm{CP}$ was comparable to recovery for $20 \mathrm{~mm}$ tumors. In some cases $\mathrm{CP}$ had significant quantitation errors in imaging the $20 \mathrm{~mm}$ tumors, especially when the tumor was mobile and the motion was large.

When respiration moves a tumor by more than the diameter of the tumor, no PET or PET/CT reconstruction method can be expected to recover the tumor's actual SUV values, unless the method accounts for the motion. In our simulation of this case, no part of the tumor at EE overlapped the tumor at EI. On the other hand, if in our simulation the total motion was less than the diameter, the bottom of the tumor at EE overlapped the top of the tumor at EI. Since we assumed that the uptake was uniform throughout the tumor, this situation creates the illusion that a piece of the tumor did not move. Because we focused our attention on $20 \mathrm{~mm}$ tumors, and because SUV values were assigned based on maximum pixel values, motion less than $15 \mathrm{~mm}$ had relatively little effect.

The poorest SUV recovery was for tumors close to the dome of the liver. We believe that this was due to large changes, over the course of the respiratory cycle, in attenuation values. The upper lobe of the liver was also strongly influenced by attenuation changes, though the effect was less than at the DOL. Quantitative errors in the lower right lung were of a smaller magnitude and we believe are mostly due to motion-blurring alone, with much smaller attenuation errors.

In many clinics where oncological PET/CT is performed today, the PET data are not gated and a CT scan is performed without special equipment or breathing instructions that would assure data acquisition at a particular, measured phase of breathing. In the case of a freely breathing patient, the phase most likely to be measured by the spiraling X-ray source is EE, because people normally pause between breaths, though the data could be acquired at any phase between EE and EI. We gave special consideration to those two extremes and in Fig. 4 plotted SUV recovery for $20-\mathrm{mm}$ tumors as a function of the extent of the five patients' diaphragmatic motion. In these cases we included a postreconstruction filter to give a more realistic estimate of the recovery achievable 
in an actual PET/CT scanner, considering spatial resolution. The partial volume effects educed uptake between $0 \%$ and $10 \%$.

Fig. 4 illustrates the complexity of respiration's effect upon PET/CT. Part (a) of the figure gives the impression that $\mathrm{CT}$ performed at EE gave correct PET/CT results, i.e. errors no larger than $20 \%$ in imaging of a mobile tumor in the lower lung, at the diaphragm, and in the upper lobe of the liver. However, part (b) shows that, again with CT performed at EE, immobile tumors at the level of the lower right lung are correctly reconstructed while tumors lower than that are overcorrected by amounts varying from $0 \%$ to $60 \%$. In fact, the pattern indicated by part (a) shows that motion blurring, which should lower reconstructed values, is canceled by an overcorrection for the attenuation. The attenuation errors are even larger near the DOL than they are in the mid chest. Part (d) of the figure gives the impression that, with CT performed at EI, the immobile tumors in the mid-chest are reconstructed rather with underestimation in the range from $0 \%$ to $30 \%$, apart from effects attributable to a $10 \%$ loss in resolution, while part (c) clearly shows that the mobile tumors, again with CT performed at EI, suffer a large undercorrection for attenuation. The pattern in part (d) is explained by the fact that much of the detected radiation from tumors in the mid chest is emitted during the part of the breathing cycle near EI when the lungs are full of air and the attenuation is least; so the EI attenuation correction works fairly well for the immobile tumors. The mobile tumors represented by part (c) move with the diaphragm, and therefore the attenuation vary less through the breathing cycle. In this case, the reconstructed tumor intensity is lowered by three phenomena: inadequate correction for attenuation; motion blurring; and a small loss of resolution. There is no canceling error in this case. When the CT scan is performed during free breathing, tumors near the diaphragm will be overcorrected or undercorrected by an amount that will not be known to the phsysician reading the images. If the CT data were acquired in an unnatural phase of breathing, for example at full inspiration to afford a good view of chest anatomy, the undercorrection for attenuation should be even larger, and lesions could be completely missed. A practical way of dealing with these problems is discussed below.

Gated CT is relatively new technology, made practical by the introduction of ever faster CT scanners with large numbers of detector rows. In our methodology for simulating a PET/CT acquisition and reconstruction system with breathing motion, gated CT volumes were converted into PET volumes and attenuation sinograms to represent the essential features of respiratory motion. This approach to studying respiration artifacts in PET/CT has benefits not easily achieved in other approaches, for example clinical studies and phantom studies. First, this approach has a gold standard, since one always compares the tumors to the known tumor introduced in the simulation. Clinical studies hardly ever have such a gold standard to compare to. Second, the motions are realistic since 
they come from actual patients. In our view, it would be very difficult to create phantom studies in which the motions are modeled with this level of realism. Since actual human tissue is used in our model of the attenuation correction, this approach even includes complex attenuation-related phenomena such as the compression of lung tissue during breathing. Third, we are able to explore the effect of respiratory motion without the confusion of other effects that are present in actual PET studies: limited counts per acquired frame; limits to the detector resolution; three instead of two-dimensional geometry; scattered radiation; and other data corrections. When we want to study motion in isolation from other effects, it is advantageous to be able to turn them off.

Our segmentation procedure had some unrealistic features. Blood in the ventricles of the heart was treated like soft tissue and clothing and blankets were sometimes treated like fat. These problems do not concern us greatly, for two reasons. First, the unrealistic effects did not occur close to the lung lesions, where the motion-related effects were large. Second, we believe that the fundamental lessons of the simulation do not depend on objects in the background.

The respiratory gates were defined at ten equally spaced intervals in the respiratory cycle. A correct physical description of the PET and AF acquisitions would involve integration over all breaths. If we assume a regular and smooth pattern of breathing, the addition of ten phases is nearly the same as time integration, in the sense that a Riemann integral can be approximated with the trapezoid rule. Our model completely ignores motion within each gate. We are not concerned by this because time integration of the motion in such a short frame, which is the appropriate physical model, is well approximated by the average value which we used, given the smooth nature of normal breathing.

The GP/GCT combination gives quantitative PET images in conditions of no statistical noise, and under the assumption that the two modalities can be matched perfectly. Clinically, none of the conditions of this simulation can be met realistically, and in addition cardiac motions in the same territory pose a separate problem that this simulation did not address. Challenges for the future include finding ways of matching the two modalities well enough to achieve quantitative accuracy in essentially every scan, especially in the presence of variable breathing patterns ${ }^{22,23}$, and finding ways to improve the statistical accuracy of images based on very low statistics per frame.

This simulation can be used to guide practitioners of oncological PET/CT to make the best possible use of existing technology in the face of respirationrelated artifacts. The mismatch between the CT and PET images leads to a number of potential problems, including localization and attenuation correction errors. Particularly when a tumor is near the diaphragm in the base of the lungs or in the superior portion of the liver, respiration may result in incorrect localization or even in the tumor being missed because of the undercorrection for attenuation. Fortunately, in a study by Osman et al., the incidence of misposi- 
tioning errors was small (less than $2 \%$ of cases) ${ }^{10}$. Nevertheless, careful attention should be given when reviewing the region near the diaphragm. The review should include PET images made without attenuation correction. Although those images are not quantitative, they are unaffected by attenuation correction errors. In the case of cooperative patients, undercorrection of attenuation due to breathing can be avoided by acquiring the CT scan of the thorax near EE. For patients who are unable to follow breathing instructions, the technologist can acquire two or three fast, low-dose CT scans during free breathing ${ }^{24}$. The appropriate $\mathrm{CT}$ scan for attenuation correction, i.e. the one that most closely corresponds to EE, is identified as the one in which the diaphragm is positioned highest. Such a procedure should reduce the likelihood that tumors near the diaphragm are missed because of respiration artifacts, and would be particularly appropriate for mobile tumors. For stationary tumors, our work indicates that quantitative imaging is more accurate when the attenuation information is derived near EI. A multi-CT protocol like the one mentioned above can also be used to select the scan in which the diaphragm is lowest. This is the breathing phase proposed by Nehmeh et al. for tumor imaging ${ }^{11}$. Therefore, existing technology provides several strategies for tumor imaging in the region near the diaphragm.

\section{SUMMARY}

We used gated CT series to provide a realistic basis for simulating errors in PET and PET/CT that are due to respiratory motion. To our knowledge this has not been done previously.

We showed that, in the conditions of the simulation, the combination of gated PET and gated CT was quantitative but other methods were not. In the majority of patients whose breathing motions are less than $15 \mathrm{~mm}$, motion-related errors are in the range of $20 \%$ or less in the case of $20 \mathrm{~mm}$ tumors. Conventional PET was not quantitative, but there were a remarkable number of cases in our simulation of $20 \mathrm{~mm}$ tumors, indeed, nearly all of them, in which canceling errors due to emission blurring and attenuation overestimation led to a nearly correct result in conventional PET, for example in Fig. 2.

We showed that a large amount of motion caused relatively large errors. Although we did not discuss it at length in this report, our simulations showed that $10 \mathrm{~mm}$ tumors were blurred to a greater extent by motion. Quantitative imaging of such tumors is in any case challenged by spatial-resolution effects, which could be larger than the motion blurring, depending on processing parameters. Conversely, $30 \mathrm{~mm}$ tumors were blurred less by motion. We demonstrated that gated PET by itself gave the correct shape for tumors, but without proper at- 
tenuation information gated PET could not be relied upon to give a quantitative image of the tumor.

The largest uptake errors occurred in tumors at the diaphragm. The upper lobe of the liver was another region where large errors occurred. The lower lung was a territory where uptake errors were smaller in most cases, presumably because the attenuation-correction errors are less while the motion blurring was the same.

Although proper alignment of the emission and transmission measurements is necessary for accurate tumor imaging near the diaphragm, the serious problem of a missed tumor can be addressed without respiratory gating, using simple CT protocols to acquire images near end expiration. By using such a protocol, tumor SUV values are overestimated by an amount in the range from $0 \%$ to $60 \%$. In cases of suspected tumors near the diaphragm, the physician should review the PET images made without attenuation.

\section{Acknowledgments}

This work arose out of discussions with Dr. T. Pan at the M. D. Anderson Cancer Center, who shared gated CT image sets with us and performed related calculations. Our understanding of the imaging of moving tumors was aided by discussions with Drs. D. Townsend and C. Nahmias at the University of Tennessee, and with our colleagues Drs. C. Michel and L. Le Meunier. The suggestion to use multiple CT scans of the chest to identify end expiration came from our cardiac PET/CT collaboration with Drs. R. Eisner and R. Patterson at Emory Crawford Long Hospital.

\section{REFERENCES}

1. G. R. Borst, J. S. Belderbos, R. Boellaard R, E. F. I. Comans, K. De Jaeger, A. A. Lammertsma, J. V. Lebesque, "Standardised FDG uptake: a prognostic factor for inoperable non-small cell lung cancer," Eur J Cancer 41 1533-41 (2005).

2. S. M. Eschmann SM, G. Friedel, F. Paulsen, M. Reimold, T. Hehr, W. Budach, J. Scheiderbauer, H. J. Machulla, H. Dittmann, R. Vonthein, R. Bares, "Is standardised 18F-FDG uptake value an outcome predictor in patients with stage III non-small cell lung cancer?" Eur $J$ Nucl Med Mol Imaging 33 263-9 (2006).

3. K. Higashi, Y. Ueda, Y. Arisaka, T. Sakuma, Y. Nambu, M. Oguchi, H. Seki, S. Taki, H. Tonami, I. Yamamoto, "18F -FDG uptake as a biologic prognostic factor for recurrence in patients with surgically resected non-small cell lung cancer," J Nucl Med 43 39-45 (2002).

4. J. F. Vansteenkiste, S. G. Stroobants, P. J. Dupont, P. R. De Leyn, E. K. Verbeken, G. J. Deneffe, L. A. Mortelmans, M. G. Demedts, "Prognostic importance of the standardized uptake value on $18 \mathrm{~F}$-fluoro-2-deoxy-glucose-positron emission tomography scan in nonsmall-cell lung cancer: an analysis of 125 cases," J Clin Oncol 17 3201-3206 (1999). 
5. A. van Baardwijk, G. Bosmans, L. Boersma, J. Buijsen, S. Wanders, M. Hochstenbag, R.J. vanSuylen, A. Dekker, C. Dehing-Oberije, R. Houben, S.M. Bentzen, M. van Kroonenburgh, P. Lambin and D. De Ruysscher, "PET-CT based auto-contouring in non-small cell lung cancer correlates with pathology and reduces interobserver variability in the delineation of the primary tumor and involved nodes," Int J Radiat Oncol Biol Phys 68(3) 771-8 (2007).

6. I. S. Grills, D. Yan, Q. C. Black, C. O. Wong, A. A. Martinez and L. L. Kestin, "Clinical implications of defining the gross tumor volume with combination of CT and 18FDGpositron emission tomography in non-small-cell lung cancer," Int J Radiat Oncol Biol Phys 67 709-719 (2007).

7. Y. Erdi, O. Mawlawi, S. M. Larson, M. Imbriaco, H. Yeung, R. Finn, J. L. Humm, "Segmentation of lung lesion volume by adaptive positron emission tomography image thresholding," Cancer 80 2505-2509 (1997).

8. U. Nestle, S. Kremp, A. Schaefer-Schuler, C. Sebastian-Welsch, D. Hellwig, C. Rübe, C. M. Kirsch, "Comparison of different methods for delineation of 18F-FDG PET-positive tissue for target volume definition in radiotherapy of patients with non-small cell lung cancer," $J$ Nucl Med 46 1342-1348 (2005).

9. P. J. Keall, G. S. Mageras, J. M. Balter, R. S. Emery, K. M. Forster, S. B. Jiang, J. M. Kapatoes, D. A. Low, M. J. Murphy, B. R. Murray, C. R. Ramsey, M. B. Van Herk, S. S. Vedam, J. W. Wong, E. Yorke, "The management of respiratory motion in radiation oncology report of AAPM Task Group 76," Med Phys 33 3874-3900 (2006).

10. M. M. Osman, C. Cohade, Y. Nakamoto, L. T. Marshall, J. P. Leal and R. L. Wahl, "Clinically significant inaccurate localization of lesions with PET/CT: frequency in 300 patients," J Nucl Med 44 240-243 (2003).

11. S. A. Nehmeh, Y. E. Erdi, G. S. P. Meirelles, O. Squire, S. M. Larson, J. L. Humm, H. Schöder, "Deep-inspiration breath-hold PET/CT of the thorax," J Nucl Med 48 22-26 (2007).

12. C. A. Nagel, G. Bosmans, A. L. A. J. Dekker, M. C. Öllers, D. K. M. De Ruysscher, P. Lambin, A. W. H. Minken, N. Lang, K. P. Schäfers "Phased attenuation correction in respiration correlated computed tomography/positron emitted tomography," Med Phys 33 18401847 (2006).

13. J. W. H. Wolthaus, M. van Herk, S. H. Muller, J. S. A. Belderbos, J. V. Lebesque, J. A. de Bois, M. M. G Rossi, E M F Damen, "Fusion of respiration-correlated PET and CT scans: correlated lung tumour motion in anatomical and functional scans," Phys Med Biol 50 1569$1583(2005)$

14. Y. E. Erdi, S. A. Nehmeh, T. Pan, A. Pevsner, K. E. Rosenzweig, G. Mageras, E. D. Yorke, H. Schoder, W. Hsiao, O. D. Squire, P. Vernon, J. B. Ashman, H. Mostafavi, S. M. Larson, J. L. Humm, "The CT motion quantitation of lung lesions and its impact on PET-measured SUVs," J Nucl Med 45 1287-1292 (2004).

15. J. J. Hamill, A. Dekker, G. Bosmans, "Respiratory-gated CT as a tool for the simulation of breathing artifacts in PET and PET/CT," abstract 539 at the Society of Nuclear Medicine 53rd annual meeting (2006).

16. X. A. Li, C. Stepaniak, E. Gore, "Technical and dosimetric aspects of respiratory gating using a pressure-sensor motion monitoring system," Med Phys 33 145-154 (2006).

17. J. P. J. Carney, D. W. Townsend, V. Rappoport, B. Bendriem, Med Phys 33 976-983 (2006).

18. J. Hamill, C. Michel, P. Kinahan, "Fast PET EM reconstruction from linograms," IEEE Trans Nucl Sci 50 1630-1635 (2004). 
19. H. H. Liu, P. Balter, T. Tutt, B. Choi, J. Zhang, C. Wang, M. Chi, D. Luo, T. Pan, S. Hunjan, G. Starkscahll, I. Rosen, K. Prado, Z. Liao, J. Chang, K. Komaki, J. D. Cox, R. Mohan, L. Dong, "Assessing respiration-induced tumor motion and internal target volume using four-dimensional computed tomography for radiotherapy of lung cancer," Int J Rad Oncol Biol Phys 68 531-540 (2007).

20. R Leahy, C Byrne, "Recent development in iterative image reconstruction for PET and SPECT," IEEE Trans Med Im 19 257-260 (editorial) (2000).

21. J Hamill, T Bruckbauer, "Iterative reconstruction methods for high-throughput PET tomographs," Phys Med Biol 47 2627-2636 (2002).

22. N. Koch, H. H. Liu, G. Starkschall, M. Jacobson, K. Forster, Z. Liao, R. Komaki, C. W. Stevens, "Evaluation of internal lung motion for respiratory-gated radiotherapy using MRI: Part I-correlating internal lung motion with skin fiducial motion" Int J Rad Oncol Biol Phys 60 1459-1472 (2004).

23. W. Lu, P. J. Parikh, J. P. Hubenschmidt, J. D. Bradley, D. A. Low, “A comparison between amplitude sorting and phase-angle sorting using external respiratory measurement for 4D CT" Med Phys 33 2964-2974 (2006).

24. J. Streeter, R. Eisner, J. Hamill, M. Nelson, R. Patterson,, "Attenuation correction of stress PET Rb82 with ultrafast CT images," abstract 1729 at the Society of Nuclear Medicine 54th annual meeting (2007). 



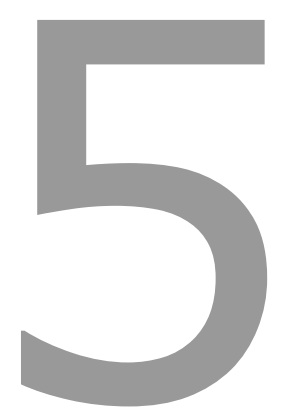

Intra-patient variability of tumor volume and tumor motion during conventionally fractionated radiotherapy for locally advanced non-small cell lung cancer. A Prospective Clinical Study

Int J Radiat Oncol Biol Phys. 2006;66(3):748-53

Geert Bosmans Angela van Baardwijk

André Dekker

Michel Öllers Liesbeth Boersma

André Minken

Philippe Lambin

Dirk De Ruysscher 


\section{ABSTRACT}

Purpose: To investigate the change in tumor volume, motion and breathing frequency during a course of radiotherapy, for locally advanced non-small cell lung cancer.

Patients and Methods: Twenty-three patients underwent CT-PET and Respiration Correlated CT scans prior to treatment, which was repeated in the first and second week following the start of radiotherapy. Patients were treated with an accelerated fractionation schedule, 1.8 Gy BID, with a total tumor dose depending on pre-set dose constraints for the lungs and the spinal cord.

Results: A striking heterogeneity of tumor volume changes was observed at all time points. In some patients the volume decreased $>30 \%(3 / 23)$ in others the volume increased $>30 \%(4 / 24)$ but for the majority of patients $(16 / 23)$ the tumor volume only changed slightly $(<30 \%)$. No significant changes in average tumor motion or breathing frequencies were seen during treatment. Although quite some changes in individual tumor motion were seen, only in one patient would this have led to an increase of the internal margin $>1 \mathrm{~mm}$ in one direction, one week after the start of treatment, and in three patients for one direction, 2 weeks after the start of the treatment.

Conclusion: A large variability of changes in tumor volume between the patients was observed. This underscores the need for repeated imaging during the course of radiotherapy. However, the changes in tumor motion are small, which indicates that repeated respiration correlated CT does not appear to be necessary. 


\section{INTRODUCTION}

Radiation therapy, alone or in combination with chemotherapy or surgery, is important in the treatment of lung cancer ${ }^{1}$. Nowadays, target volume delineation is often based on sophisticated imaging techniques such as CT and FDGPET scans, as this improves the diagnostic accuracy and may allow radiation dose escalation ${ }^{2-5}$. Breathing induced tumor motion and hence appropriate margins can be taken into account using 4D-CT information from respiration correlated CT (RC-CT) scans. Incorporating this temporal information in radiotherapy planning could also lead to a more accurate delineation of the gross tumor volume. In general, smaller margins can be applied which would make dose escalation or a decrease of normal tissue exposure possible ${ }^{6}$.

However, radiation treatment planning is based on imaging taken at a single time-point before the start of radiotherapy, and therefore any changes of tumor motion, tumor volume or breathing frequency during the course of fractionated radiotherapy are not incorporated in the treatment planning process.

Few data on volume changes during radiotherapy for lung cancer patients have been reported ${ }^{7-10}$, showing conflicting results. Moreover, the evolution of the tumor motion during radiotherapy was only reported in stereotactic radiotherapy for stage I NSCLC patients ${ }^{10}$, although this information is important for Adaptive RadioTherapy (ART).

We therefore investigated the changes of tumor volume, tumor motion and breathing frequency during the first 2 weeks of an accelerated course of radiotherapy in patients with locally advanced non-small cell lung cancer (NSCLC).

\section{PATIENTS AND METHODS}

\section{Patients}

Twenty-three patients with histological or cytological proven non-small cell lung cancer (NSCLC) (UICC stage II-III) were included in this study, from December 2004 until September 2005. Twenty patients received three cycles of carboplatin and gemcitabine chemotherapy before radiotherapy, had a WHO performance status $0-1$ and a good lung function (FEV1 $\geq 50 \%$ and DLCO $\geq$ $50 \%$ ). Three patients did not receive chemotherapy due to medical reasons. The response to induction chemotherapy was evaluated using the RECIST-criteria

${ }^{11}$. No concurrent chemotherapy was given. Patients were treated using our current standard CT-PET simulation, radiation treatment planning, and fractionation schedule, as described below.

The Medical Ethics Committee according to the Dutch law approved the trial. All patients gave written informed consent before entering this study. 


\section{Radiotherapy simulation}

Patients were simulated in radiotherapy position on a dedicated CT-PETsimulator with both arms above the head and on an immobilization and patient laser marker system. Radiotherapy technologists trained specifically for this purpose always carried out the simulation. The CT-PET scanner used was a Siemens Biograph (SOMATOM Sensation-16 with an ECAT ACCEL PET scanner).

An intravenous injection of ${ }^{18}$ F-Fluoro-Deoxy-Glucose (FDG) (Tyco Health Care, Amsterdam, The Netherlands) of (weight $\times 4+20$ ) MBq was followed by $10 \mathrm{ml}$ physiologic saline. After a 45 minutes uptake period, during which the patient was encouraged to rest, PET and CT images were acquired. A spiral CT with intravenous contrast was performed covering the complete thoracic region.

\section{Radiotherapy planning}

Radiotherapy planning was performed on a XiO (Computerized Medical Systems,St Louis, Missouri) treatment planning system, using inhomogeneity corrections, based on a convolution algorithm. For all patients, the Gross Tumor Volume (GTV) and the Planning Target Volume (PTV) were defined, based on CT-PET data, obtained prior to radiotherapy (i.e. post chemotherapy in case of inducton chemotherapy). The Clinical Target Volume (CTV) was defined as the GTV with a $5 \mathrm{~mm}$ margin incorporating microscopic disease. This CTV was subsequently expanded with $1 \mathrm{~cm}$ to draw the PTV to incorporate the internal respiratory motion and setup errors.

For the volume comparison in this study, the CT-GTV was defined as the primary tumor on lung window $(\mathrm{W}=1600, \mathrm{~L}=-800)$.

Contouring of the lungs was carried out automatically by the treatment planning system. For the calculation of the mean lung dose (MLD), the volume of both lungs excluding the GTV was used ${ }^{12}$. The spinal cord was drawn throughout the whole CT scan and was considered to be at the inner margin of the bony spinal canal.

A 3D conformal treatment plan was calculated on the PTV for all patients according to ICRU 50 guidelines ${ }^{13}$. Dosimetric values were calculated on the basis of dose-volume histograms and dose distributions on each axial CT plan.

\section{Radiotherapy dose and normal tissue constraints}

For each patient, the radiation dose was escalated to a mean lung dose (MLD) of 19 Gy while respecting a maximum spinal cord dose of $54 \mathrm{~Gy}$. There were no esophageal dose constraints. As the tumor dose was delivered in 1.8 Gy fractions, a maximum deviation of the constraints of $\pm 4 \%$ was allowed. Radiother- 
apy was always delivered twice a day (BID), with a minimum of 8 hours between the two fractions, and given 5 days per week.

\section{Respiration Correlated CT scans (RC-CT)}

For the patients who gave informed consent for this study, the CT-PET was immediately followed by a respiration correlated $\mathrm{CT}$, using offline correlation of the 16-slice spiral CT scan of the CT-PET system. This had been modified to enable a low pitch (minimum 0.1 ), in combination with a respiratory signal obtained by a pressure sensor in a chest belt (AZ-733V, Anzai Medical Corporation, Tokyo, Japan). The raw CT data were reconstructed in 10 phases, from $0 \%$ to $100 \%$ in steps of $10 \%$ of the respiration period starting at end inspiration. Inhouse developed software with Matlab (Mathworks Inc, Natick, MA) was used to measure the motion of the lesion in all three orthogonal directions.

\section{Study design}

During the course of radiotherapy, the patients underwent a repeat CT-PET scan and a respiration correlated $\mathrm{CT}$ in treatment position, one and two weeks after the start of treatment. At all time points, the primary tumor was delineated using the free-breathing CT rather than on a single phase of a respiration correlated CT, as respiration correlated CT data was not available in all patients (see Table 3 ). This approach assumes that no blurring of the moving tumor occurs in the free-breathing CT. This assumption was checked and found to be valid as the high-speed 16-slice CT scan protocol, effectively "freezes" the tumor in a single phase during a free-breathing CT. The tumor was delineated for each patient by the same physician to avoid interobserver variability. To study the intraobserver variability, all pre-treatment CT-scans were delineated twice by the same physician, as was the case for ten patients for all time points, with at least a 3 month time interval.

Due to technical and logistical problems, a complete image dataset at all three time points, was not available for all patients. We missed a CT-PET dataset for one patient one week after the start of treatment and for three patients, two weeks after the start of treatment.

\section{Endpoints and statistical analysis}

We determined the tumor volumes prior to 1 and 2 weeks, after start of the treatment, based on the GTVs drawn on the free-breathing CT. In addition, we used the respiration correlated CT to quantify the tumor motion at all 3 time points. Tumor motion was expressed as a $3 \mathrm{D}$ vector ${ }^{14}$, which is the quadratic sum of the motions in the three orthogonal directions. Finally, breathing frequency was recorded at all three time-points during the respiration correlated 
CT. All results are expressed as the mean \pm standard deviation (SD). Statistical differences between paired parameters from the three time points were evaluated using the Wilcoxon signed rank test. Differences were considered to be significant when the p-value was less than 0.05 .

\section{RESULTS}

\section{Patient characteristics}

The patient characteristics are shown in Table 1 . The mean values of the total tumor dose (TTD) and the corresponding mean lung dose (MLD) were 61.7 Gy \pm 9.8 Gy [range: 46.8 Gy -79.2 Gy] and 16.4 Gy \pm 3.4 Gy [range: 6.8 Gy $20.8 \mathrm{~Gy}$, respectively. The GTV of the primary tumor before radiotherapy was $87.5 \mathrm{~cm}^{3} \pm 137.7 \mathrm{~cm}^{3}$ [range: $0.4 \mathrm{~cm}^{3}-660.2 \mathrm{~cm}^{3}$ ].

The first repeat scan was made after the patient received on average a dose of 20.4 Gy \pm 4.1 Gy (about 11 fractions) and the second scan after 38.3 Gy \pm 5.1 Gy (about 21 fractions) (Table 2).

\section{Volume changes of the primary tumor}

A striking heterogeneity of the changes in volume of the primary tumor during radiotherapy was observed. Using an arbitrarily chosen cut-off level of $30 \%$, four patients $(17 \%)$ showed an increase of the tumor volume of $>30 \%$, for sixteen patients $(70 \%)$ the volume change varied $<30 \%$ while for three patients $(13 \%)$ the volume decreased $>30 \%$. In Fig. 1 these patients are indicated with a dotted line for the patients with a large increase in tumor volume, a dashed line for those patients with a large decrease and a solid line for the rest of the patients.

Although the average volumes do not reflect the individual changes, we observed a significant increase in average tumor volume in the patients $(n=22)$ for which data at the first and second time point was available, from $90.9 \mathrm{~cm}^{3} \pm$ $139.9 \mathrm{~cm}^{3}$ to $99.2 \mathrm{~cm}^{3} \pm 130.4 \mathrm{~cm}^{3}$ from the start of radiation to week $1(\mathrm{p}=$ 0.049). For time point at week 2 , we missed the CT information for three patients. In the remaining 20 patients the tumor decreased on average from 94.7 $\mathrm{cm}^{3} \pm 146.3 \mathrm{~cm}^{3}$ to $86.4 \mathrm{~cm}^{3} \pm 109.8 \mathrm{~cm}^{3}$ from the start of radiation to week $2(\mathrm{p}$ $=0.940$ ) (Table 2 and Fig. 2).

No significant difference in tumor volume changes was observed between patients with stable disease and partial response to induction chemotherapy.

For the 43 delineations, which were performed twice, we found an average intra-observer variability of $1.2 \% \pm 4.4 \%$, with a maximum deviation of $9.5 \%$. 


\begin{tabular}{|c|c|c|c|c|c|c|c|c|}
\hline $\begin{array}{l}\text { Patient } \\
\text { no. }\end{array}$ & $\begin{array}{l}\text { Age } \\
\text { (years) }\end{array}$ & Gender & Stage & $\begin{array}{l}\text { Location } \\
\text { of the pri- } \\
\text { mary tu- } \\
\text { mor }\end{array}$ & $\begin{array}{l}\text { Re- } \\
\text { sponse } \\
\text { chemo- } \\
\text { therapy }\end{array}$ & $\begin{array}{l}\text { TTD } \\
\text { (Gy) }\end{array}$ & $\begin{array}{l}\text { MLD } \\
\text { (Gy) }\end{array}$ & $\begin{array}{l}\text { OTT } \\
\text { (days) }\end{array}$ \\
\hline 1 & 52 & $\mathrm{~F}$ & T1N3M0 & RLL & PR & 54.0 & 17.6 & 17 \\
\hline 2 & 54 & M & $\mathrm{T} 3 \mathrm{~N} 2 \mathrm{M} 0$ & RML & SD & 54.0 & 19.3 & 20 \\
\hline 3 & 75 & M & $\mathrm{T} 3 \mathrm{~N} 2 \mathrm{M} 0$ & RUL & PR & 54.0 & 15.2 & 20 \\
\hline 4 & 71 & $\mathrm{~F}$ & T3N1M0 & RUL & no & 64.8 & 20.7 & 23 \\
\hline 5 & 71 & M & $\mathrm{T} 2 \mathrm{~N} 3 \mathrm{M} 0$ & RUL & PR & 54.0 & 14.7 & 23 \\
\hline 6 & 61 & M & T1N3M0 & RML & PR & 68.4 & 14.9 & 22 \\
\hline 7 & 71 & M & $\mathrm{T} 2 \mathrm{~N} 3 \mathrm{M} 0$ & LLL & PR & 54.0 & 16.0 & 19 \\
\hline 8 & 77 & $\mathrm{M}$ & T4N2M0 & RUL & PR & 61.2 & 17.6 & 22 \\
\hline 9 & 74 & $\mathrm{~F}$ & T2N0M0 & RLL & no & 54.0 & 16.3 & 20 \\
\hline 10 & 79 & $\mathrm{~F}$ & T4N0M0 & RLL & PR & 57.6 & 18.3 & 23 \\
\hline 11 & 57 & $\mathrm{M}$ & T1N3M0 & LUL & PR & 64.8 & 8.5 & 27 \\
\hline 12 & 68 & M & T4N0M0 & RUL & PR & 54.0 & 6.8 & 20 \\
\hline 13 & 63 & M & T4N0M0 & RLL & SD & 64.8 & 17.3 & 24 \\
\hline 14 & 59 & $\mathrm{~F}$ & $\mathrm{~T} 2 \mathrm{~N} 2 \mathrm{M} 0$ & RML & PR & 46.8 & 20.0 & 16 \\
\hline 15 & 55 & M & T4N2M0 & LUL & SD & 79.2 & 16.4 & 29 \\
\hline 16 & 52 & $\mathrm{~F}$ & $\mathrm{~T} 2 \mathrm{~N} 3 \mathrm{M} 0$ & LLL & PR & 54.0 & 15.6 & 20 \\
\hline 17 & 50 & $\mathrm{~F}$ & T4N2M0 & LLL & PR & 61.2 & 20.8 & 21 \\
\hline 18 & 71 & $\mathrm{M}$ & T4N2M0 & RUL & PR & 79.2 & 13.6 & 29 \\
\hline 19 & 61 & $\mathrm{~F}$ & $\mathrm{~T} 2 \mathrm{~N} 2 \mathrm{M} 0$ & RML & PR & 57.6 & 19.0 & 21 \\
\hline 20 & 62 & $\mathrm{M}$ & $\mathrm{T} 2 \mathrm{~N} 2 \mathrm{M} 0$ & RUL & PR & 68.4 & 18.8 & 27 \\
\hline 21 & 80 & $\mathrm{M}$ & T2N0M0 & LLL & no & 61.2 & 15.2 & 21 \\
\hline 22 & 56 & $\mathrm{M}$ & T4N2M0 & LUL & SD & 79.2 & 17.8 & 30 \\
\hline 23 & 54 & M & $\mathrm{T} 2 \mathrm{~N} 3 \mathrm{M} 0$ & LUL & SD & 79.2 & 16.1 & 30 \\
\hline Mean & 64.0 & & & & & 61.7 & 16.4 & 22.8 \\
\hline SD & 9.6 & & & & & 9.8 & 3.4 & 4.1 \\
\hline \multicolumn{9}{|c|}{$\begin{array}{l}\text { TTD = Total Tumor Dose; MLD = Mean Lung Dose; OTT = Overall Treatment Time } \\
\text { F = Female; M = Male; PR = Partial Remission as response to induction chemotherapy; SD = } \\
\text { Stable Disease as response to induction chemotherapy (RECIST criteria) } \\
\text { RLL: Right Lower Lobe; LLL: Left Lower Lobe; RUL: Right Upper Lobe:LUL: Left Upper } \\
\text { Lobe; RML: Right Middle Lobe }\end{array}$} \\
\hline
\end{tabular}


Table 2: Evolution of the Gross Tumor Volume of the primary tumor during radiotherapy.

\begin{tabular}{|c|c|c|c|c|c|}
\hline $\begin{array}{l}\text { Patient } \\
\text { no. }\end{array}$ & $\begin{array}{l}\text { Volume } \\
\text { before RT } \\
\text { (cm3) }\end{array}$ & $\begin{array}{c}\text { Corresponding } \\
\text { dose after one } \\
\text { week of RT (Gy) }\end{array}$ & $\begin{array}{c}\text { Volume } \\
\text { after one } \\
\text { week of RT } \\
(\mathrm{cm} 3)\end{array}$ & $\begin{array}{c}\text { Corresponding } \\
\text { dose after two } \\
\text { weeks of RT (Gy) }\end{array}$ & $\begin{array}{l}\text { Volume } \\
\text { after two } \\
\text { weeks of } \\
\text { RT (cm3) }\end{array}$ \\
\hline 1 & 7.9 & 19.8 & 4.7 & 37.8 & 5.2 \\
\hline 2 & 660.2 & 18.0 & 580.3 & 34.2 & 455.9 \\
\hline 3 & 53.1 & 18.0 & 65.9 & NA & NA \\
\hline 4 & 26.6 & 19.8 & 14.7 & 37.8 & 2.8 \\
\hline 5 & 97.3 & 18.0 & 155.0 & 36.0 & 134.6 \\
\hline 6 & 7.5 & 19.8 & 4.3 & 34.2 & 5.0 \\
\hline 7 & 28.1 & 16.2 & 43.8 & 37.8 & 36.4 \\
\hline 8 & 3.5 & 19.8 & 5.9 & 41.4 & 6.0 \\
\hline 9 & 64.9 & 27.0 & 35.8 & NA & NA \\
\hline 10 & 12.2 & NA & NA & 36.0 & 10.8 \\
\hline 11 & 0.4 & 19.8 & 0.5 & NA & NA \\
\hline 12 & 63.2 & 23.4 & 64.6 & 37.8 & 72.3 \\
\hline 13 & 67.2 & 19.8 & 88.6 & 48.6 & 42.2 \\
\hline 14 & 272.2 & 23.4 & 323.8 & 41.4 & 288.9 \\
\hline 15 & 92.9 & 19.8 & 104.3 & 37.8 & 84.0 \\
\hline 16 & 79.0 & 21.6 & 99.7 & 39.6 & 83.8 \\
\hline 17 & 92.9 & 19.8 & 153.5 & 37.8 & 121.1 \\
\hline 18 & 7.3 & 21.6 & 6.2 & 32.4 & 6.4 \\
\hline 19 & 101.9 & 16.2 & 131.2 & 41.4 & 80.1 \\
\hline 20 & 24.2 & 19.8 & 28.0 & 37.8 & 26.7 \\
\hline 21 & 107.7 & 14.4 & 114.7 & 27 & 105.3 \\
\hline 22 & 73.0 & 18 & 75.9 & 39.6 & 65.0 \\
\hline 23 & 69.0 & 34.2 & 79.9 & 50.4 & 95.8 \\
\hline Mean & 87.5 & 20.4 & 99.2 & 38.3 & 84.4 \\
\hline SD & 137.7 & 4.1 & 130.4 & 5.1 & 109.8 \\
\hline
\end{tabular}

$p=0.049$ one week vs prior; $p=0.007$ two weeks vs one week; $p=0.940$ two weeks vs prior RT: Radiotherapy

NA: Not Assessed because of technical problems 


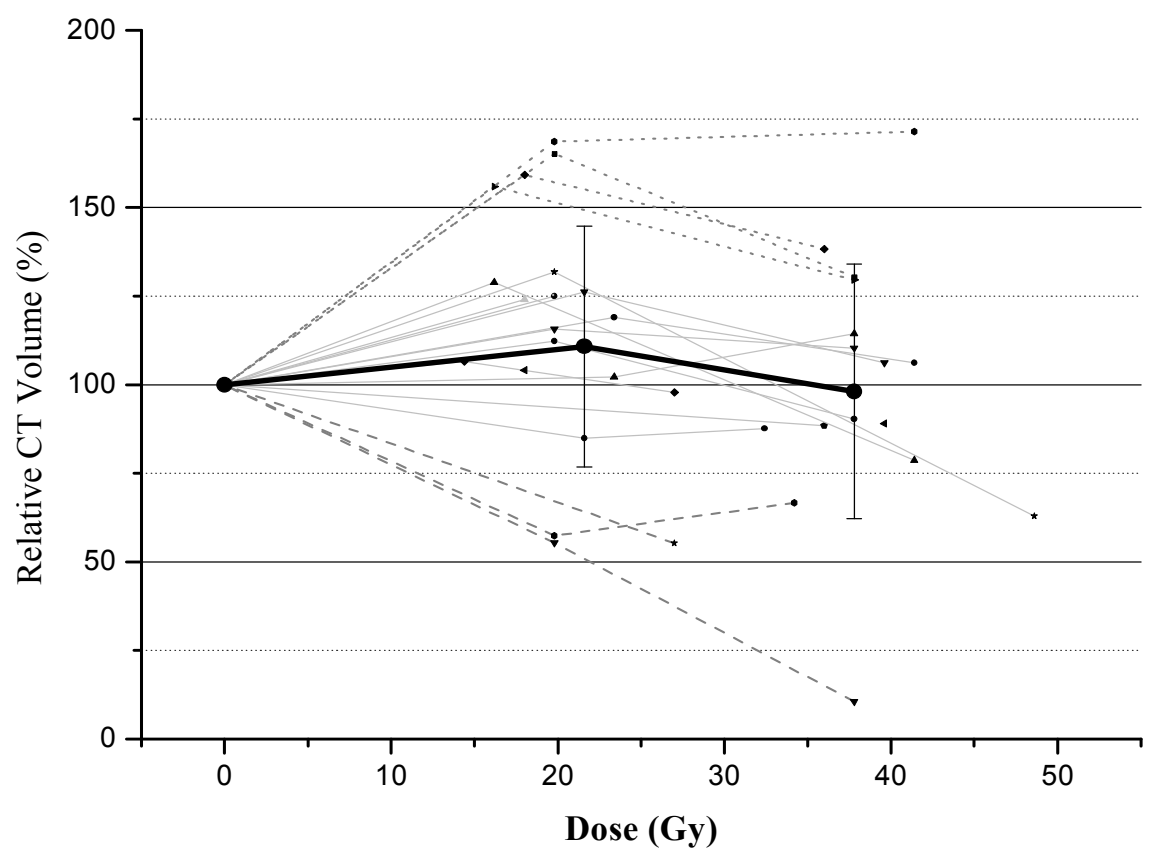

Figure 1: The CT volumes, relative to the volume prior to treatment, were plotted versus the dose. The thick black line is the average relative CT volume together with the standard deviation. The dotted lines represents the patients where the tumor increased more than $30 \%$, the dashed lines those patients with a decrease of more than $30 \%$.

\section{Changes of the motion of the primary tumor}

Tumor motion, expressed as a $3 \mathrm{D}$ vector, which is the quadratic sum of the motion in the three orthogonal directions and the breathing frequency are shown in Table 3 and Fig. 3. On average, the total vector motion changed from $7.8 \mathrm{~mm} \pm$ $4.8 \mathrm{~mm}$ initially to $7.8 \mathrm{~mm} \pm 5.9 \mathrm{~mm}$ and $8.0 \mathrm{~mm} \pm 5.6 \mathrm{~mm}$ one and two weeks after the start of the radiotherapy respectively. All differences compared to the initial tumor motion were not statistically different from each other $(p=0.969$ and $\mathrm{p}=0.582$ respectively).

When we looked at the individual differences for the motion for all directions compared to the initial motion, we observed a maximal absolute difference of $4.5 \mathrm{~mm}$ and $6.4 \mathrm{~mm}$ one and two weeks after the start of radiotherapy. The maximal difference between week 2 and week 1 after the start of radiotherapy was $5.4 \mathrm{~mm}$. In Fig 3, a histogram of these differences is shown.

If we use the peak to peak amplitude divided by four, as used for the internal margin in van Herk's margin recipe ${ }^{15}$, only for one patient, for one direction the 
internal margin increased more than $1 \mathrm{~mm}$ one week after the start of radiotherapy. Two weeks after radiotherapy the internal margin should have been changed for three patients with more than $1 \mathrm{~mm}$ (Fig. 3).

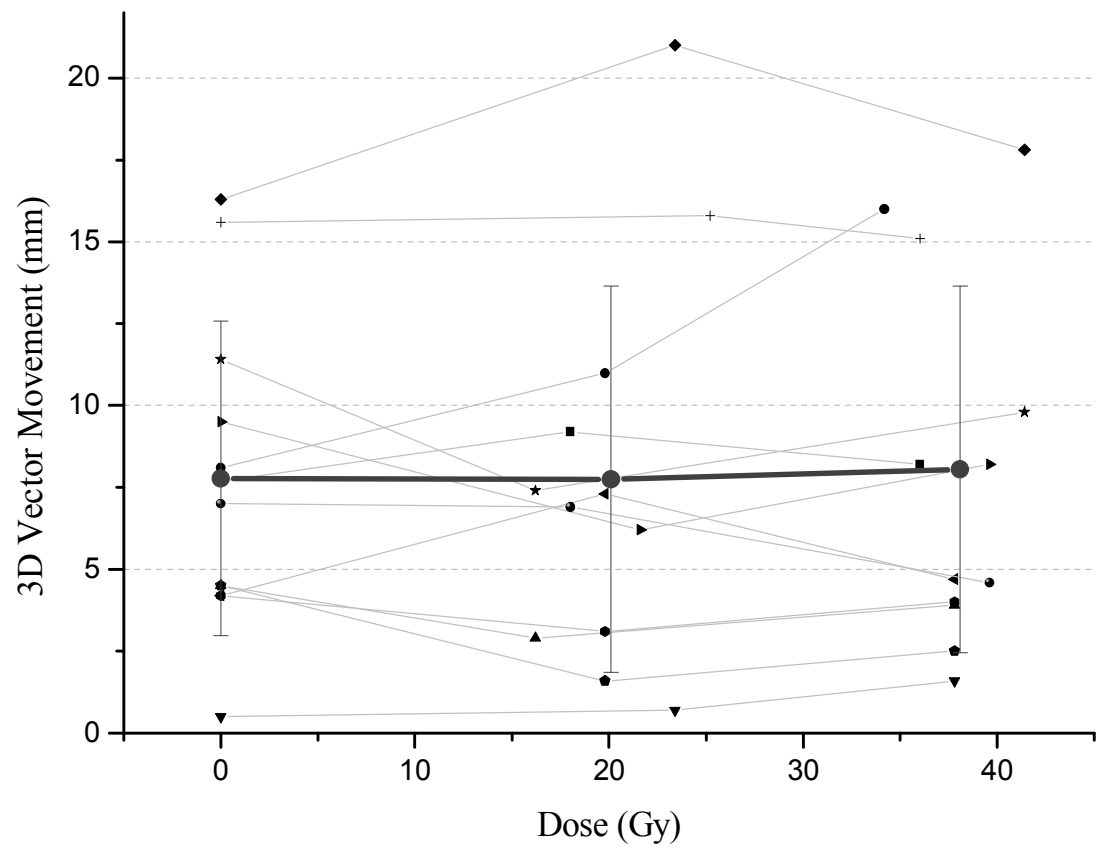

Figure 2: 3D Vector motion, which is the quadratic sum of the motion in three orthogonal directions, plotted versus the dose. The thick black line represents the average tumor motion, together with the standard deviation.

\section{Change of the breathing frequency}

The breathing frequency was not statistically different at the three time points. On average, the frequency changed from $18.5 \pm 3.6$ breathing cycles per minute (BPM) to $19.2 \pm 4.1 \mathrm{BPM}$ and $18.3 \pm 4.5 \mathrm{BPM}(\mathrm{p}=0.800$ and $\mathrm{p}=0.880$ respectively).

\section{DISCUSSION}

For high precision radiation therapy, it is important to have, during a course of radiation therapy, knowledge of the changes of several parameters, such as the tumor volume and tumor motion. 


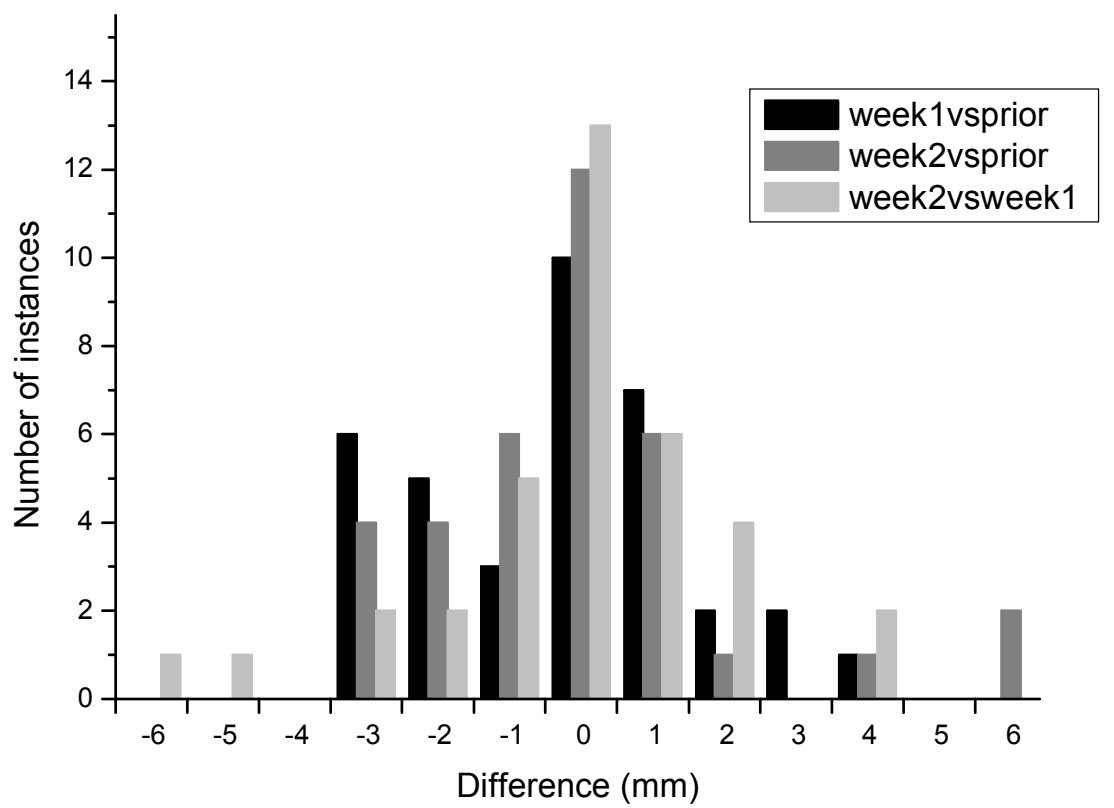

Figure 3: Histogram of the differences $(\mathrm{mm})$ in motion amplitude between all time points. The differences for all three orthogonal directions are shown.

We observed a large heterogeneity of the changes in tumor volume in our patient population. Only a few patients $(3 / 23)$ showed a large systematic decrease $(>30 \%)$ in tumor volume, whereas in the majority of patients $(16 / 23)$, the tumor volume only slightly changed $(<30 \%)$. We even found a large increase in tumor volume for four out of 23 patients. The clinical impact of these volume changes and how to adapt treatment is however still unclear. Further clinical and planning studies need to be done to clarify this problem.

So, in contrast to other investigators ${ }^{7,8}$, no significant decrease in the tumor volume during the first two weeks of radiotherapy was found. Several differences between the reported studies and our study may explain the differences. In contrast to the present study, in the studies of Kupelian et al. ${ }^{7}$ and Erridge et $a l .{ }^{8}$, patients were treated with a conventional fractionation scheme (2 Gy/day) delivered over an overall treatment time of more than 40 days. In our study, all patients received an accelerated schedule in which 1.8 Gy per fraction was delivered BID, 5 days per week. It may be that the decrease of the tumor volume by radiation-induced cancer cell death was offset by treatment-induced inflammation, which once again increased the volume. The net result may be a stable volume. Comparable results in tumor volume changes on CT scans in the first two weeks due to radiotherapy have been described when high doses per frac- 
tions (7.5 Gy - 20 Gy) are used in stereotactic radiotherapy for stage I NSCLC 9,10 . We may get more insight in the internal tumor processes by analyzing the FDG distribution in the tumor during the course of radiation. Unfortunately it is still too early to correlate these changes in tumor volume with outcome. These analyses will however be the subject of another paper.

\begin{tabular}{|c|c|c|c|c|c|c|}
\hline \multirow[b]{2}{*}{ Patient no. } & \multicolumn{2}{|c|}{ Before RT } & \multicolumn{2}{|c|}{ After one week of RT } & \multicolumn{2}{|c|}{ After two weeks of RT } \\
\hline & 3D Vector & BPM & 3D Vector & BPM & 3D Vector & BPM \\
\hline 5 & 7.7 & 22.2 & 9.2 & 22.2 & 8.2 & 25.5 \\
\hline 6 & 8.1 & 15.5 & 11.0 & 12.0 & 16.0 & 17.6 \\
\hline 7 & 4.5 & 24.0 & 2.9 & 23.1 & 3.9 & 21.6 \\
\hline 12 & 0.5 & 14.0 & 0.7 & 15.0 & 1.6 & 12.6 \\
\hline 14 & 16.3 & 17.5 & 21.0 & 17.6 & 17.8 & 19.0 \\
\hline 15 & 4.2 & 12.9 & 7.3 & 23.5 & 4.7 & 17.1 \\
\hline 16 & 9.5 & 19.2 & 6.2 & 25.5 & 8.2 & 21.1 \\
\hline 17 & 4.2 & 21.6 & 3.1 & 21.6 & 4.0 & 24.5 \\
\hline 19 & 11.4 & 22.2 & 7.4 & 20.3 & 9.8 & 16.1 \\
\hline 20 & 4.5 & 16.9 & 1.6 & 16.3 & 2.5 & 16.7 \\
\hline 21 & 15.6 & 19.7 & 15.8 & 17.9 & 15.1 & 17.9 \\
\hline 22 & 7.0 & 16.0 & 6.9 & 15.4 & 4.6 & 9.9 \\
\hline MEAN & 7.8 & 18.5 & 7.8 & 19.2 & 8.0 & 18.3 \\
\hline SD & 4.8 & 3.6 & 5.9 & 4.1 & 5.6 & 4.5 \\
\hline $\begin{array}{l}\text { 3D Vector: } \\
\text { BPM: } p=0 \\
\text { BPM: Brea }\end{array}$ & $\begin{array}{l}=0.969 \text { one } \\
9 \text { one week } \\
\text { a cycles Per }\end{array}$ & $\begin{array}{l}\text { k vs pri } \\
\text { rior; } p \\
\text { inute }\end{array}$ & $\begin{array}{l}=0.582 \text { two } \\
875 \text { two wee }\end{array}$ & $\begin{array}{l}\text { ks vs } 1 \\
\text { prior }\end{array}$ & & \\
\hline
\end{tabular}

To the best of our knowledge, this is the first study dealing with the changes of the motion and the breathing frequency of patients with locally advanced (Stage II-III) NSCLC during a course of radiotherapy. Underberg et al. ${ }^{10}$ described similar findings for Stage I NSCLC treated with hypofractionated stereotactic radiotherapy. Although we found no significant changes, on average, in tumor motion and breathing frequency, during the first two weeks of radiotherapy, in some patients quite a wide variation was seen. However, even for the largest difference in tumor motion, the clinical implication on the size of the PTV and hence on the treatment fields is limited, since only a fraction of this motion is used for PTV expansion to account for the tumor motion. Only for one patient, for one direction the internal margin increased more than $1 \mathrm{~mm}$ one week after the start of radiotherapy. Two weeks after radiotherapy the internal margin should have been changed for three patients with more than $1 \mathrm{~mm}$ (Fig. 3). In 
this patient group, the respiration correlated CT information will not influence the internal margin that needs to be taken.

In conclusion, our results clearly show that, at least when an accelerated radiotherapy schedule is given, changes in the volume of the primary tumor do occur during the first two weeks of radiotherapy. A large variability among the patients was observed, indicating that repeated imaging during the course of radiotherapy is necessary to know the changes in GTV and to be able to individualize radiation treatment. In contrast, repeated respiration correlated CT imaging during radiotherapy does not appear to be necessary as the changes in tumor motion are small, and will not lead to a change in treatment.

\section{REFERENCES}

1. De Ruysscher D, Wanders R, Boersma L, et al. Radiotherapy in the management of lung cancer. International Journal of Respiratory Care 2005; Winter:1-5.

2. van Der Wel A, Nijsten S, Hochstenbag M, et al. Increased therapeutic ratio by 18FDG-PET CT planning in patients with clinical CT stage N2-N3M0 non-small-cell lung cancer: a modeling study. Int J Radiat Oncol Biol Phys 2005;61:649-655.

3. De Ruysscher D, Wanders S, Minken A, et al. Effects of radiotherapy planning with a dedicated combined PET-CT-simulator of patients with non-small cell lung cancer on dose limiting normal tissues and radiation dose-escalation: a planning study. Radiother Oncol 2005;77:5-10.

4. De Ruysscher D, Wanders S, van Haren E, et al. Selective mediastinal node irradiation based on FDG-PET scan data in patients with non-small-cell lung cancer: a prospective clinical study. Int J Radiat Oncol Biol Phys 2005;62:988-994.

5. Bradley J, Graham MV, Winter K, et al. Toxicity and outcome results of RTOG 9311: a phase I-II dose-escalation study using three-dimensional conformal radiotherapy in patients with inoperable non-small-cell lung carcinoma. Int J Radiat Oncol Biol Phys 2005;61:318328.

6. Bosmans G, Buijssen J, Dekker A, et al. An "in silico" clinical trial comparing free breathing, slow and respiratory correlated computed tomography in lung cancer patients. Radiother Oncol 2005;76:S92.

7. Kupelian PA, Ramsey C, Meeks SL, et al. Serial megavoltage CT imaging during external beam radiotherapy for non-small-cell lung cancer: Observations on tumor regression during treatment. Int J Radiat Oncol Biol Phys 2005;63:1024-1028.

8. Erridge SC, Seppenwoolde Y, Muller SH, et al. Portal imaging to assess set-up errors, tumor motion and tumor shrinkage during conformal radiotherapy of non-small cell lung cancer. Radiother Oncol 2003;66:75-85.

9. Senan S, Underberg R, Slotman B, et al. Time trends in target volumes during stereotactic radiotherapy for Stage I non-small cell lung cancer (NSCLC). Int J Radiat Oncol Biol Phys 2004;60:S281-S282.

10. Underberg R, Lagerwaard F, van Tinteren H, et al. Time trends in target volumes for stage I non-small-cell lung cancer after stereotactic radiotherapy. Int J Radiat Oncol Biol Phys 2006; 64(4):1221-8. 


\section{0 | Chapter 5}

11. Therasse P, Arbuck SG, Eisenhauer EA, et al. New guidelines to evaluate the response to treatment in solid tumors. European Organization for Research and Treatment of Cancer, National Cancer Institute of the United States, National Cancer Institute of Canada. J Natl Cancer Inst 2000;92:205-216.

12. Kwa SL, Lebesque JV, Theuws JC, et al. Radiation pneumonitis as a function of mean lung dose: an analysis of pooled data of 540 patients. Int J Radiat Oncol Biol Phys 1998;42:1-9.

13. ICRU report 62: Prescribing, recording, and reporting photon beam therapy (Supplement to ICRU report 50). Bethesda, MD: International Commission on Radiation Units and Measurements. 1999.

14. van Sornsen de Koste JR, Lagerwaard FJ, Nijssen-Visser MR, et al. Tumor location cannot predict the mobility of lung tumors: a 3D analysis of data generated from multiple CT scans. Int J Radiat Oncol Biol Phys 2003;56:348-354.

15. van Herk M. Errors and margins in radiotherapy. Semin Radiat Oncol 2004;14:52-64. 
C H A P T E R

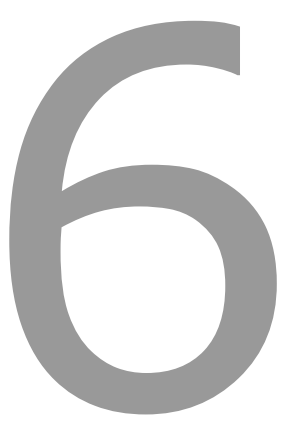

Time trends in the maximal uptake of FDG on PET scan during thoracic radiotherapy. A prospective study in locally advanced nonsmall cell lung cancer (NSCLC) patients

Radiother Oncol. 2007;82(2):145-52

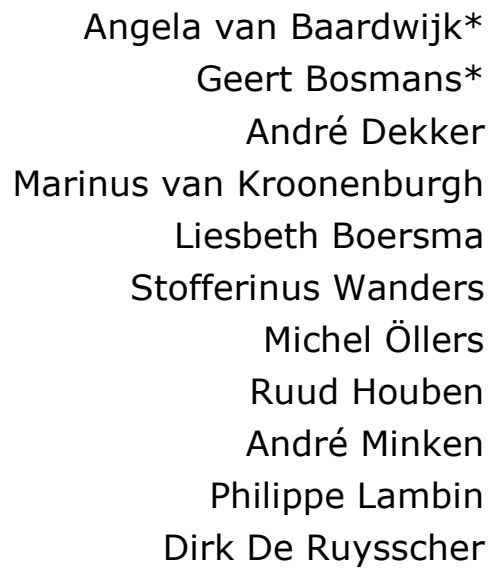

* these authors equally contributed to this study 
92 | Chapter 6

\begin{abstract}
Background and purpose: ${ }^{18} \mathrm{~F}$-fluoro-2-deoxy-glucose (FDG) uptake on PET scan is a prognostic factor for outcome in NSCLC. We investigated changes in FDG uptake during fractionated radiotherapy in relation to metabolic response with the ultimate aim to adapt treatment according to early response.

Methods and materials: Twenty-three patients, medically inoperable or with advanced NSCLC, underwent four repeated PET-CT scans before, during and after radiotherapy. Changes in maximal standardized uptake value $\left(\mathrm{SUV}_{\max }\right)$ were described. Patients were treated with accelerated radiotherapy with a total tumor-dose depending on normal tissue dose constraints.

Results: The most striking result was the large intra-individual heterogeneity in the evolution of $\mathrm{SUV}_{\max }$. For the total group a non-significant increase in the first week $(p=0.05)$, and a decrease in the second week $(p=0.02)$ and after radiotherapy $(\mathrm{p}<0.01)$ was observed. Different time trends were shown for responders (no change during radiotherapy) and non-responders (48\% increase during first week, $\mathrm{p}=0.02$ and $15 \%$ decrease in the second week, $\mathrm{p}=0.04$ ). Non-responders had a higher $\mathrm{SUV}_{\max }$ on all time points investigated.

Conclusions: Time trends in $\mathrm{SUV}_{\max }$ showed a large intra-individual heterogeneity and different patterns for metabolic responders and non-responders. These new findings may reflect intrinsic tumor characteristics and might finally be useful to adapt treatment.
\end{abstract}




\section{INTRODUCTION}

Thoracic irradiation plays an important role in the management of advanced non-small cell lung cancer (NSCLC). Although the prognosis of these patients has improved, local tumor control is still not achieved in the majority of the patients ${ }^{37,44,45}$. Local tumor control can be improved by increasing the total radiation dose and the addition of chemotherapy to radiotherapy, but normal tissue toxicity, like esophagitis and radiation pneumonitis, is dose limiting $3,11,20,30,34,38$. A more individualized prediction of response to a specific therapy is needed in order to avoid needless treatment or to be able to propose an alternative therapy if possible ${ }^{29,42,46}$. Methods to describe tumor characteristics include imaging techniques, like positron emission tomography (PET). In different tumor sites pre- and post-treatment PET scans have been correlated to outcome ${ }^{19,24,57}$. Indeed, the maximal uptake of 18F-fluoro-2-deoxy-glucose (FDG) in the primary lung tumor as measured on a PET-scan before treatment was consistently shown to be a significant, independent, prognostic factor for survival in NSCLC, both in case of surgical resection and radical radiotherapy ${ }^{1,5,15,18,25,53}$. On the other hand, a post-treatment PET scan can predict residual disease and hence outcome in NSCLC ${ }^{9,26,32,33,56}$. Although there is a paucity on data dealing with the evolution of FDG uptake during fractionated radiotherapy, it is logical to study these time trends as an early predictive parameter, as has been shown for chemotherapy ${ }^{12,14,16,22,26}$. We hypothesized that changes in uptake might reflect early tumor response during radiotherapy, which might finally allow further individualization and adaptation of treatment before therapy is completed ${ }^{7}$.

The aim of the current study was to describe changes in the uptake of FDG on PET scans during a course of radiotherapy in patients with NSCLC and to investigate whether there are differences in time trends in FDG uptake between metabolic responders and non-responders.

\section{METHODS AND MATERIALS}

\section{Study population}

From December 2004 to September 2005, 23 patients with medically inoperable or locally advanced NSCLC were enrolled in a phase I study. Patients with localized, histologically confirmed NSCLC, a WHO-performance score of 0 or 1 , a weight loss of $<10 \%$ in 6 months and a reasonable lung function (a FEV1 > $50 \%$ of predicted value and a DLCO $\geq 50 \%$ ) were eligible. In case of previous chemotherapy, radiotherapy was started after a minimum of 14 days after the last chemotherapy course. The response to induction chemotherapy was evalu- 
ated using the RECIST-criteria ${ }^{48}$. No previous radiotherapy to the chest was allowed. Patients were not eligible if they had any other concomitant malignant disease, a recent severe cardiac disease or active peptic esophagitis.

\section{PET-CT-scan}

As part of this phase I study repeated PET-CT scans (before start of radiotherapy, on day 7 and day 14 during radiotherapy and 70 days after radiotherapy) were performed in radiotherapy position on a dedicated PET-CT-simulator with both arms above the head and using a laser marker system. The PET-CT scanner used was a Biograph ${ }^{\mathrm{TM}}$ (SOMATOM Sensation 16 with its ECAT ACCEL PET scanner, Siemens). Patients were fasting for at least $6 \mathrm{~h}$ before the examination. FDG (MDS Nordion, Liege, Belgium) was injected intravenously and followed by physiologic saline $(10 \mathrm{ml})$. The injected total activity of FDG depended on the weight of the patient: (weight $\times 4+20$ ) MBq. After a rest period of 45 min (time needed for uptake of FDG) PET and CT images were acquired. The CT-scan performed was a spiral CT-scan of the whole thorax, with intravenous contrast. The PET images were acquired in 5-min bed positions, while 2 or 3 positions were needed to scan the thoracic region. The standardized uptake value (SUV) was used to measure the amount of uptake of FDG in the primary tumor. A region of interest (ROI) was drawn on the 3D-images in E-soft (version 5.0.24.5, Siemens, Erlangen, Germany) around the region of the primary tumor. SUV was calculated as activity concentration of FDG uptake divided by injected dose/body weight ${ }^{49}$. To avoid partial volume effects as much as possible, the maximal SUV ( $\mathrm{SUV}_{\max }$ ) within this ROI was calculated and used for correlations.

\section{Treatment description}

All patients enrolled in this phase I study were irradiated using an individualized prescribed maximal tolerated dose (MTD). This total tumor dose (TTD) was based on normal tissue constraints instead of a classic fixed prescribed dose. For the lungs a mean lung dose (MLD) of 19.0 Gray (Gy) and for the spinal cord a maximal dose of 54.0 Gy were used as dose constraints. The maximal allowed TTD was 79.2 Gy or lower if any of the normal dose constraints were reached. The margin from GTV (gross tumor volume) to CTV (clinical target volume) was $5 \mathrm{~mm}$ and for the PTV (planning target volume) another $10 \mathrm{~mm}$ for the primary tumor and $5 \mathrm{~mm}$ for lymph nodes were added. No elective nodal irradiation was carried out. A 3D conformal treatment plan, using three to six coplanar $10 \mathrm{MV}$ photon fields, was calculated according to the ICRU guidelines 27. Calculations were performed using an $\mathrm{XiO}$ treatment planning system (Computer Medical System Inc.) with a FFT convolution algorithm for inhomogeneity corrections ${ }^{35}$. Patients were irradiated on a linear accelerator (Elekta SL 15, 
Crawley, United Kingdom). Radiotherapy was delivered twice daily, with at least an 8-h interval (BID scheme) and with a fraction size of 1.8 Gy. Results concerning toxicity and outcome of this phase I study will be reported separately.

\section{Assessment of metabolic response}

Metabolic response was measured 70 days after radiotherapy by means of a PET-CT scan. This scan was compared to the pre-treatment scan, following the recommendations of the EORTC (Table 1) ${ }^{58}$. The total group was divided in responders, showing complete metabolic response (CMR) or partial metabolic response (PMR), and non-responders with stable metabolic disease (SMD) or progressive metabolic disease (PMD).

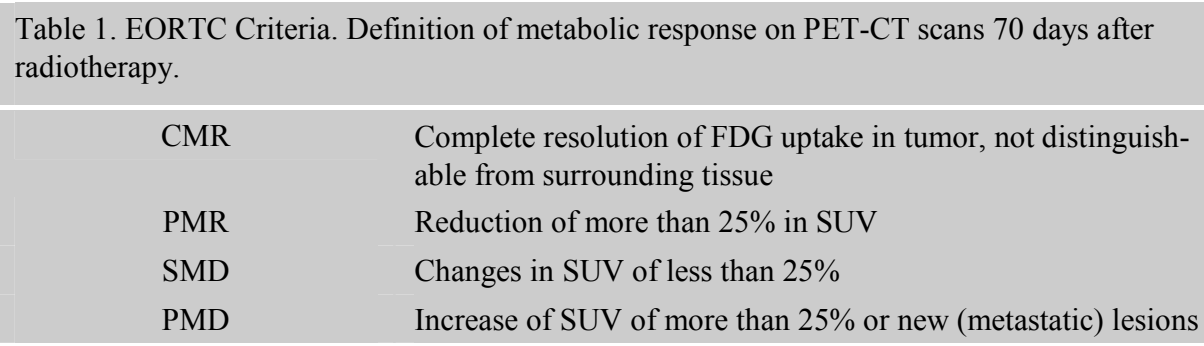

EORTC: European Organization for Research and Treatment of Cancer; CMR: complete metabolic response; PMR: partial metabolic response; SMD: stable metabolic disease; PMD: progressive metabolic disease; SUV: standardized uptake value.

\section{Statistics}

The SPSS software (SPSS for Windows, version 14.0, SPSS Inc., Chicago, IL) was used for statistical analysis. Results are expressed as mean \pm SD (standard deviation) for individual results and as mean \pm SE (standard error of the mean) for groups. The paired Student's t-test was used to compare the $\mathrm{SUV}_{\max }$ on different time points, whereas the Mann-Whitney U-test and the Fisher's exact test was used to compare the different groups. Follow-up was updated and frozen at July 1st of 2006. Survival estimates were calculated using the Kaplan-Meier method and comparison of the survival curves were made using the log-rank test. Patients lost to follow-up were censored from survival data. Two-tailed pvalues are provided and p-values less than 0.05 were considered statistically significant. 


\section{Ethics}

This study has been reviewed and approved by the local Medical Ethical Committee, according to the Dutch law and regulations. All patients gave written informed consent before entering this study.

\section{RESULTS}

\section{Patient characteristics}

The patient characteristics are depicted in Table 2. Three patients did not receive chemotherapy, while 20 patients received platinum-based chemotherapy (Cisplatin or Carboplatin, combined with Gemcitabin). As a result of induction chemotherapy 15 patients showed a partial remission and 5 patients showed stable disease. The median interval between the last course of chemotherapy and the start of radiotherapy was $35 \pm 16$ days (range: $14-56$ days). The average total tumor dose (TTD) given was 62.5 \pm 9.7 Gy (range: 46.8-79.2 Gy) in an overall treatment time (OTT) of 22 days (range: 17-30 days). The first PET-CT scan was performed before start of radiotherapy (median 9 days before start of radiotherapy, range 4-13 days), the second on day 7 of radiotherapy (at a median dose of $19.8 \pm 4.1 \mathrm{~Gy}$ ) and the third on day 14 of radiotherapy (at a median dose of $37.8 \pm 5.1 \mathrm{~Gy}$ ). The fourth PET-CT scan was performed 70 days after the last day of radiotherapy (median: $71 \pm 12$ days). Because of logistic reasons or technical problems with the PET-scan, no PET information was available for 1 patient on day 7 and for 4 patients on day 14 of radiotherapy. For 3 patients no PET scan was available after radiotherapy, in one because of technical reasons and in 2 patients because of early death after radiotherapy due to a myocardial infarction and a pulmonary infection. The median follow-up (FU) from start of RT was $9.2 \pm 3.8$ months.

\section{Time trends in maximal SUV}

There was a large heterogeneity in the changes in $\mathrm{SUV}_{\max }$ of the primary tumor during the radiation treatment between patients (Fig. 1). Out of the 22 patients who had a PET-CT at day 7 of radiotherapy, 4 patients (18\%) showed a decrease of more than $25 \%$ of the $\mathrm{SUV}_{\max }$, whereas $41 \%(9 / 22)$ showed an increase of more than $25 \%$. The remainder $(9 / 22)$ showed a stable $\mathrm{SUV}_{\max }(<25 \%$ change) compared to pre-treatment values. 


\begin{tabular}{|c|c|c|c|c|c|c|c|c|}
\hline $\begin{array}{l}\text { patient } \\
\text { no. }\end{array}$ & $\begin{array}{l}\text { age } \\
\text { (yr) }\end{array}$ & sex & cTNM & pathology & $\begin{array}{l}\text { response to } \\
\text { induction } \\
\text { chemo- } \\
\text { therapy }\end{array}$ & $\begin{array}{l}\text { OTT } \\
\text { (days) }\end{array}$ & $\begin{array}{l}\text { TTD } \\
\text { (Gy) }\end{array}$ & $\begin{array}{l}\text { Metabolic } \\
\text { response } \\
70 \text { days } \\
\text { after RT }\end{array}$ \\
\hline 1 & 52 & $\mathrm{f}$ & T1N3M0 & adenoca & PR & 21 & 54.0 & CMR \\
\hline 2 & 54 & $\mathrm{~m}$ & T3N2M0 & Large cell ca & $\mathrm{SD}$ & 21 & 54.0 & PMD \\
\hline 3 & 75 & $\mathrm{~m}$ & T3N2M0 & large cell ca & PR & 21 & 54.0 & no scan \\
\hline 4 & 71 & $\mathrm{f}$ & T3N1M0 & $\mathrm{scc}$ & no chemo & 28 & 64.8 & CMR \\
\hline 5 & 71 & $\mathrm{~m}$ & $\mathrm{~T} 2 \mathrm{~N} 3 \mathrm{M} 0$ & adenoca & PR & 21 & 54.0 & $\dagger$ \\
\hline 6 & 61 & $\mathrm{~m}$ & T1N3M0 & scc & PR & 27 & 64.8 & CMR \\
\hline 7 & 71 & $\mathrm{~m}$ & $\mathrm{~T} 2 \mathrm{~N} 3 \mathrm{M} 0$ & adenoca & PR & 20 & 60.0 & PMD \\
\hline 8 & 77 & $\mathrm{~m}$ & T4N2M0 & large cell ca & $\mathrm{PR}$ & 22 & 61.2 & $\mathrm{CMR}$ \\
\hline 9 & 74 & $\mathrm{f}$ & T2N0M0 & large cell ca & no chemo & 20 & 54.0 & PMR \\
\hline 10 & 79 & $\mathrm{f}$ & T4N0M0 & large cell ca & PR & 24 & 57.6 & PMR \\
\hline 11 & 57 & $\mathrm{~m}$ & T1N3M0 & large cell ca & PR & 30 & 64.8 & CMR \\
\hline 12 & 68 & $\mathrm{~m}$ & T4N0M0 & adenoca & PR & 21 & 54.0 & CMR \\
\hline 13 & 63 & $\mathrm{~m}$ & T4N0M0 & $\mathrm{scc}$ & $\mathrm{SD}$ & 24 & 64.8 & SMD \\
\hline 14 & 59 & $\mathrm{f}$ & $\mathrm{T} 2 \mathrm{~N} 2 \mathrm{M} 0$ & large cell ca & $\mathrm{PR}$ & 17 & 46.8 & PMD \\
\hline 15 & 55 & $\mathrm{~m}$ & T4N2M0 & adenoca & $\mathrm{SD}$ & 30 & 79,2 & PMD \\
\hline 16 & 52 & $\mathrm{f}$ & T2N3M0 & $\mathrm{scc}$ & PR & 21 & 54.0 & PMD \\
\hline 17 & 50 & $\mathrm{f}$ & T4N2M0 & large cell ca & $\mathrm{PR}$ & 22 & 61.2 & PMD \\
\hline 18 & 71 & $\mathrm{~m}$ & T4N2M0 & large cell ca & PR & 30 & 79.2 & PMR \\
\hline 19 & 61 & $\mathrm{f}$ & $\mathrm{T} 2 \mathrm{~N} 2 \mathrm{M} 0$ & large cell ca & PR & 21 & 57.6 & SMD \\
\hline 20 & 62 & $\mathrm{~m}$ & $\mathrm{~T} 2 \mathrm{~N} 2 \mathrm{M} 0$ & large cell ca & PR & 27 & 68.4 & $\mathrm{CMR}$ \\
\hline 21 & 80 & $\mathrm{~m}$ & T2N0M0 & $\operatorname{scc}$ & no chemo & 22 & 61.2 & $\dagger$ \\
\hline 22 & 56 & $\mathrm{~m}$ & T4N2M0 & large cell ca & $\mathrm{SD}$ & 30 & 79.2 & PMR \\
\hline 23 & 54 & $\mathrm{~m}$ & T2N3M0 & large cell ca & SD & 30 & 79.2 & PMD \\
\hline \multicolumn{9}{|c|}{$\begin{array}{l}\mathrm{scc}=\text { squamous cell carcinoma, } \mathrm{PR}=\text { partial remission as response to induction chemotherapy, } \\
\mathrm{SD}=\text { stable disease as response to induction chemotherapy, OTT = overall treatment time of } \\
\text { radiotherapy, } \mathrm{TTD}=\text { total tumour dose, } \mathrm{CMR}=\text { complete metabolic response, } \mathrm{PMR}=\text { partial } \\
\text { metabolic response, } \mathrm{SMD}=\text { stable metabolic disease, } \mathrm{PMD}=\text { progressive metabolic disease. } \\
\dagger=\text { patient died within first } 70 \text { days after radiotherapy. }\end{array}$} \\
\hline
\end{tabular}

For the whole patient group, the $\mathrm{SUV}_{\max }$ was on average $7.3 \pm 0.8$ (range: 0.616.1) before start of radiotherapy, which increased to $8.9 \pm 1.2$ (range: 1.3-23.1) on day 7 of treatment $(p=0.05)$. In the second week, a significant decrease was observed to a $\mathrm{SUV}_{\max }$ of $8.1 \pm 1.0$ (range: $\left.2.1-17.8\right)(\mathrm{p}=0.02)$. Seventy days after treatment the $\mathrm{SUV}_{\max }$ further decreased to a mean level of $4.8 \pm 3.3$ (range: 
1.2-13.4), a significant decrease compared to day 14 of treatment $(\mathrm{p}=0.001)$ and compared to pre-treatment levels $(\mathrm{p}=0.005)$ (Fig. 2a). Analysing the subset of patients $(\mathrm{n}=16)$, who received a PET-CT scan at all time points during radiotherapy, similar results were obtained: $\mathrm{SUV}_{\max }$ before radiotherapy $7.0 \pm 0.9$ and at day $79.7 \pm 1.5(\mathrm{p}=0.02)$, day $148.0 \pm 1.1(\mathrm{p}=0.02)$ and 70 days after radiotherapy $4.7 \pm 0.8(\mathrm{p}=0.002)$.

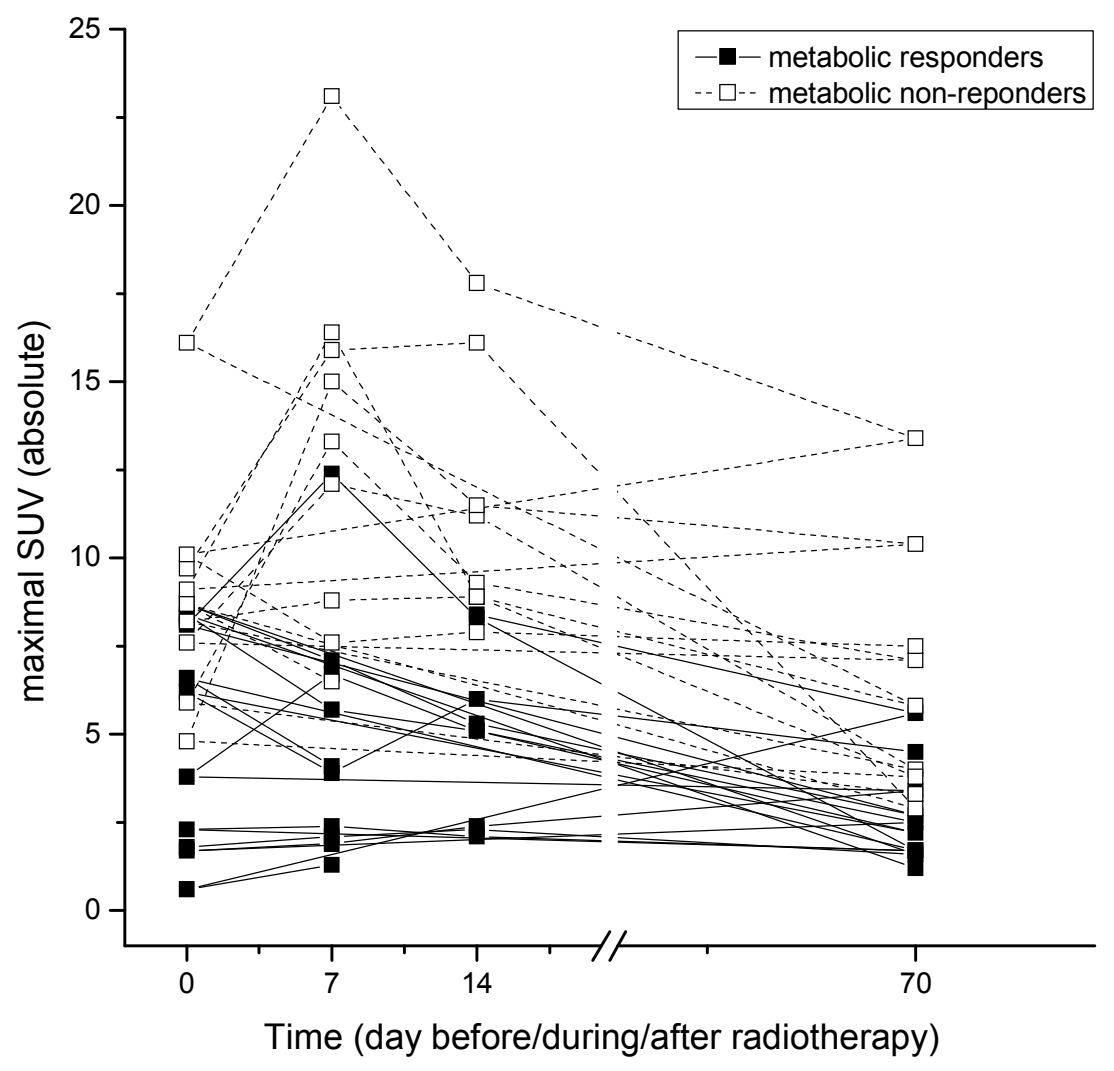

Figure 1. The absolute (maximal SUV (standardized uptake value) plotted versus time (before start RT, day 7 and day 14 during RT and 70 days after RT) for all individual patients with a CTPET scan at day 70.

\section{Metabolic responders versus non-responders}

In 20 patients a PET-CT scan was performed 70 days after treatment. In 1 patient (with visually a CMR) no $\mathrm{SUV}_{\max }$ could be calculated, due to a software problem. Eleven patients (55\%) showed a metabolic response 70 days after ra- 
diotherapy [7 patients CMR and 4 patients PMR], while 9 patients (45\%) showed no response [2 patients SMD, 7 patients PMD]. Concerning the PMD group, 4 patients had metastatic disease and 3 patients showed loco-regional progression.
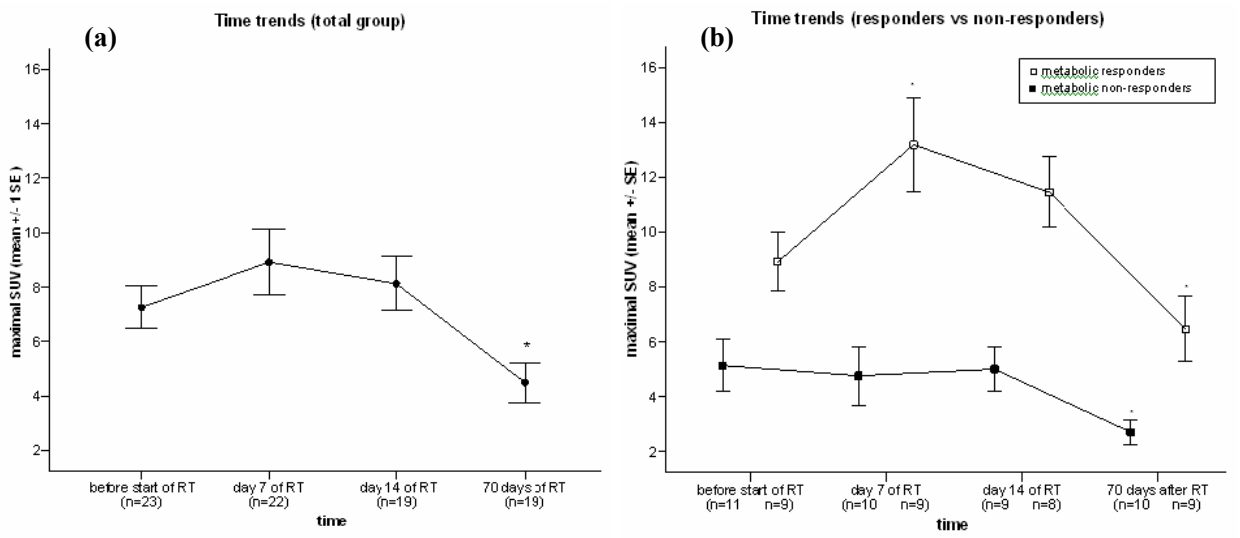

Figure 2. Average change in maximal SUV for the total group of patients (a) and for metabolic responders (complete and partial metabolic response) versus non-responders (stable metabolic disease and progressive metabolic disease) (b), * indicates a significant difference.

Patients with a metabolic response 70 days after thoracic irradiation had a significantly longer overall survival (estimated survival of $100 \%$ after 9 months of follow-up) compared to the patients with stable or progressive disease (estimated survival of $63 \%$ after 9 months of follow-up) $(p=0.005)$ (Fig. 3). No difference was observed between total radiation dose prescribed $(p=0.4)$ and the overall treatment time of radiotherapy $(p=0.2)$ between metabolic responders and non-responders.

In the group of metabolic responders no change in $\mathrm{SUV}_{\max }$ was observed during the course of radiotherapy. Only after radiotherapy a mean decrease of $46 \%$ of the $\mathrm{SUV}_{\text {max }}$ was observed from $5.0 \pm 0.8$ on day 14 during radiotherapy to $2.7 \pm$ 0.470 days after radiotherapy ( $p=0.02$; Fig. $2 b$, Table 3 ). In contrast to this finding, the group of non-responders showed a significant increase of the SU$\mathrm{V}_{\max }$ in the first week of radiotherapy. In this group the $\mathrm{SUV}_{\max }$ before treatment was on average $8.9 \pm 1.1$ and increased to $13.2 \pm 1.7$ in the first week $(p=0.02)$. In the second week a decrease was observed to an average level of $11.5 \pm 1.3(\mathrm{p}$ $=0.04)$, with a further decrease after radiotherapy to a mean of $6.9 \pm 1.2(\mathrm{p}=$ 0.02). Similar results were obtained for the subset of patients with a PET-CT scan on day 0,7 and 14: for the responders $\mathrm{SUV}_{\max }$ did not change during radio- 
therapy (before radiotherapy, $5.1 \pm 1.1$; day $7,5.3 \pm 4.6 \pm 0.8$; day $14,4.6 \pm$ 0.8 , while for the non-responders the $\mathrm{SUV}_{\max }$ increased during the first week and decreased again during the second week of radiotherapy (before radiotherapy, $8.9 \pm 1.2$; day $7,14.0 \pm 1.7(\mathrm{p}=0.01)$ and day $14,11.5 \pm 1.3(\mathrm{p}=0.04)$.

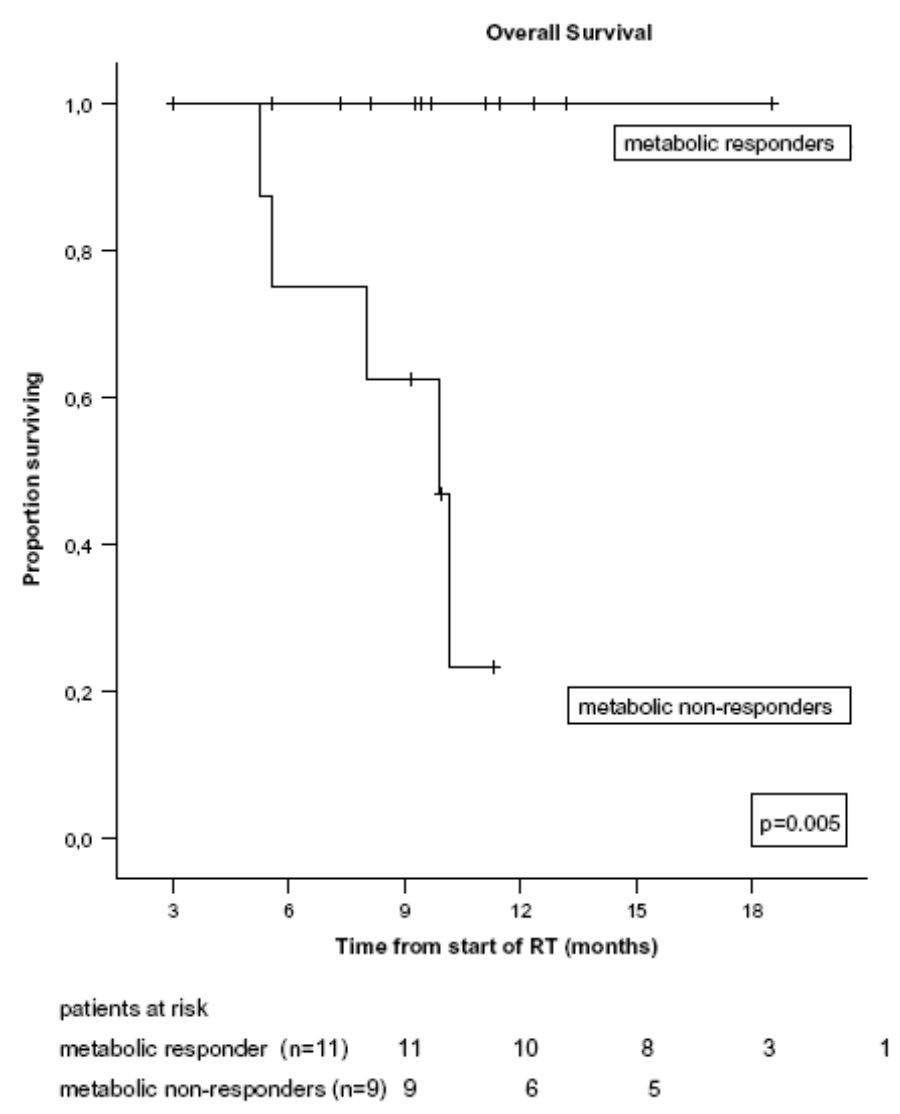

Figure 3. Actuarial analysis of overall survival (Kaplan-Meier) in months for metabolic responders $(n=11)$ and non-responders $(n=9)$, classified according to EORTC-criteria. p-value of log-rank test.

Not only a difference in time trends in $\mathrm{SUV}_{\max }$ was observed between responders and non-responders, but the non-responders also showed a significant higher $\mathrm{SUV}_{\max }$ than the responders at all time points investigated (Fig. 2, Table 3).

The number of patients in this study was however too small to test the predictive value of the time trends in $\mathrm{SUV}_{\max }$ in relation to metabolic response. 


\section{Influence of induction chemotherapy}

In total 20 patients received induction chemotherapy, of which 18 patients were evaluable 70 days after irradiation. The group of patients achieving a partial remission after induction chemotherapy (13/18) showed in higher percentage metabolic response after radiotherapy (metabolic response rate, 62\%) as patients showing stable disease as a result of the induction chemotherapy (metabolic response rate, $20 \%)$, although this difference was not significant $(p=0.3)$. The time patterns of the $\mathrm{SUV}_{\max }$ in relation to metabolic response as described above for the total group were similar in patients having received induction chemotherapy.

This excludes the possibility that the 3 individuals, who did not receive systematic treatment, would have influenced the overall results.

\begin{tabular}{|c|c|c|c|c|c|c|c|c|c|c|}
\hline \multirow[b]{2}{*}{$\begin{array}{l}\text { Maximal } \\
\text { SUV }\end{array}$} & \multicolumn{3}{|c|}{ Total group } & \multicolumn{3}{|c|}{$\begin{array}{l}\text { Metabolic } \\
\text { responders }\end{array}$} & \multicolumn{3}{|c|}{$\begin{array}{c}\text { Metabolic } \\
\text { non-responders }\end{array}$} & \multirow[b]{2}{*}{$\mathrm{p}$} \\
\hline & mean & SE & $(\mathrm{N})$ & mean & SE & $(\mathrm{N})$ & mean & SE & $(\mathrm{N})$ & \\
\hline $\begin{array}{l}\text { Before start } \\
\text { of radiother- } \\
\text { apy }\end{array}$ & 7.3 & 0.8 & (23) & 5.1 & 0.9 & (11) & 8.9 & 1.1 & (9) & 0.020 \\
\hline $\begin{array}{l}\text { Day } 7 \text { of } \\
\text { radiotherapy }\end{array}$ & 8.9 & 1.2 & (22) & 4.8 & 1.1 & (10) & 13.2 & 1.7 & (9) & 0.001 \\
\hline $\begin{array}{l}\text { Day } 14 \text { of } \\
\text { radiotherapy }\end{array}$ & 8.1 & 1.0 & (19) & 5.0 & 0.8 & (9) & 11.5 & 1.3 & (8) & $<0.001$ \\
\hline $\begin{array}{l}70 \text { days after } \\
\text { radiotherpy }\end{array}$ & 4.5 & 0.7 & (19) & 2.7 & 0.4 & (10) & 6.5 & 1.2 & (9) & 0.002 \\
\hline
\end{tabular}

\section{DISCUSSION}

Until now the PET-scan is mainly used before start of radiotherapy for staging purposes, to delineate the primary tumor and/or mediastinal lymph nodes and after therapy to assess the remission status $2,6,13,17,21,32,33,39,50,52,54$. Similar to early response measurements during chemotherapy, FDG-PET scans performed in the beginning of radiotherapy might give information on the later tumor response 
$14,22,26,41$. However, only limited data on this subject are available in the literature ${ }^{12,16}$. We therefore assessed in the present study the time trends of FDG uptake during radiation treatment in NSCLC.

A striking result was the large heterogeneity in the $\mathrm{SUV}_{\max }$ in serial PET-scans between the individual patients. While some patients showed an increase, others show a stable or decreased FDG uptake after 1 week of radiation. This confirms the findings of Erdi et al., who described serial PET-scans during radiation therapy in 2 NSCLC patients ${ }^{16}$. De Geus-Oei et al. investigated repeated FDG-PET scans in patients treated with accelerated radiotherapy with carbogen and nicotinamide (ARCON) for head-and-neck cancer and also showed very heterogeneous changes in $\mathrm{SUV}_{\max }$ after hyperoxygenation with a median interval of 2 days between the two scans ${ }^{12}$. Investigating the time trends we observed for the total group a trend in increase of the $\operatorname{SUV}_{\max }(\mathrm{p}=0.05)$ during the first week of radiotherapy, followed by a significant decrease in the second week of treatment and in the first 70 days after treatment. Only few other authors described repeated (CT)-PET scans during radiation therapy. Geets et al. described reimaging with FDG-PET scan during radiation treatment in head-and-neck patients ${ }^{23}$. After an average dose of $46 \mathrm{~Gy}, 18$ patients received a repeat PETscan, which was hard to interpret due to inflammation.

In our patients with NSCLC we did not encounter this problem. de Geus-Oei et al. showed in 22 patients with head and-neck tumors a median decrease of relative change of $\mathrm{SUV}_{\max }$ of $4 \%$, comparing the PET scan after hyperoxygenation to the baseline scan ${ }^{12}$. Lin et al. described repeated PET-scans during treatment in cervical patients, but no results concerning the (maximal) SUV were investigated ${ }^{31}$. In both the study of Lin and Geets, most patients received concurrent chemo-radiation, while in the study of de Geus-Oei et al. patients were treated with ARCON.

By using the EORTC criteria to evaluate the metabolic response after 70 days we could confirm the findings of Mac Manus et al. that metabolic responders had a better overall survival than the non-responders $(p=0.005)^{33}$. Moreover, a significant difference in the time trends of $\mathrm{SUV}_{\max }$ during radiotherapy between metabolic responders and non-responders was found. Metabolic non-responders had a higher $\mathrm{SUV}_{\max }$ at all time points investigated and showed a significant increase in $\mathrm{SUV}_{\max }$ during the first week of irradiation followed by a decrease. In contrast, the responders showed a stable $\mathrm{SUV}_{\max }$ during irradiation. The number of included patients was however too small to calculate the predictive value of the changes in $\mathrm{SUV}_{\max }$ during a course of radiation. The question thus remains what causes the observed time trends and their relation with subsequent response. A first hypothesis is that the increase of $\mathrm{SUV}_{\max }$ during the first week is due to inflammation, since a rather high median dose of 19.8 Gy (1.8 Gy BID) was already delivered at the time point of the first repeat PET-CT scan. Radiation is known to be a potent inducer of inflammation in several tissues 
${ }^{28,43,51}$. However, this cannot explain the differences in metabolic responders and non-responders, since one would also expect inflammatory changes in the group of responders. Another possibility is that the $\mathrm{SUV}_{\max }$ provides information related to intrinsic biological characteristics of the tumor, such as tumor hypoxia. As hypoxia is strongly related to therapy resistance and the occurrence of metastases, this hypothesis is particularly attractive. Hypoxia might play a role in the uptake of FDG, since it leads via the hypoxia inducible factor-1 $\alpha$ (HIF-1 $\alpha)$ pathway to up regulation of glucose transporters. However, contradictory findings concerning the relation between FDG uptake and hypoxia have been published ${ }^{8,10,36,40,47,55,59,60}$.

Although the radiation dose (range: 46.8-79.2 Gy) could have influenced the response rate, a dose-response relation could not be established in this series, presumably due to the limited number of patients ${ }^{11,34}$. Another possibility is that the response on induction chemotherapy may also affect the subsequent response to radiation. However, this could not be shown in our study. To fully exclude the influence of induction chemotherapy it would have been best to perform the first PET-CT scan before start of any treatment. Although most of our patients underwent a PET-(CT) scan before start of chemotherapy, most of these scans were performed at different PET-(CT) scanners in affiliated hospitals. Since maximal SUV of different scanners cannot be compared directly, we were not able to encompass maximal SUV before start of chemotherapy into our results.

Lastly, the overall treatment time of radiotherapy might influence response after radiotherapy ${ }^{4}$. We did not find such a correlation between OTT of radiotherapy and the metabolic response rate, but this may be due to a short OTT (less than 5 weeks) in all patients. Our present working hypothesis is that the difference in time trends of $\mathrm{SUV}_{\max }$ as observed between responders and non-responders might reflect a complex relationship between several factors, like changes in blood flow as well as changes in the extracellular compartment, together with intrinsic tumor properties, which all contribute to the uptake of the FDG tracer. Further in-depth studies, including analysis of several plasma markers at different time points, to address this question are ongoing.

In conclusion, a large heterogeneity in changes in $\mathrm{SUV}_{\max }$ was observed during thoracic radiation. Differences in time trends of $\mathrm{SUV}_{\max }$ during radiotherapy were found between metabolic responders and non-responders. Further research addressing the question whether these time trends can predict response, the biological meaning of the different time trends and whether it finally might enable individualized adjustments of treatment is ongoing. The final goal, being prediction of response early during the course of radiotherapy to enable early adjustments, like prescribing a higher total dose, adjusting the fractionation scheme or adding another form of treatment. 


\section{REFERENCES}

1. Ahuja V, Coleman RE, Herndon J, et al. The prognostic significance of fluorodeoxyglucose positron emission tomography imaging for patients with nonsmall cell lung carcinoma. Cancer 1998;83:918-24.

2. Ashamalla H, Rafla S, Parikh K, et al. The contribution of integrated PET/CT to the evolving definition of treatment volumes in radiation treatment planning in lung cancer. Int $J$ Radiat Oncol Biol Phys 2005;63:1016-23.

3. Belderbos J, Heemsbergen W, Hoogeman M, et al. Acute esophageal toxicity in non-small cell lung cancer patients after high dose conformal radiotherapy. Radiother Oncol 2005;75:157-64.

4. Bentzen SM, Thames HD. Clinical evidence for tumor clonogen regeneration: interpretations of the data. Radiother Oncol 1991;22:161-6.

5. Borst GR, Belderbos JS, Boellaard R, et al. Standardised FDG uptake: a prognostic factor for inoperable non-small cell lung cancer. Eur J Cancer 2005;41:1533-41.

6. Bradley J, Thorstad WL, Mutic S, et al. Impact of FDGPET on radiation therapy volume delineation in non-smallcell lung cancer. Int J Radiat Oncol Biol Phys 2004;59:78-86.

7. Brahme A. Biologically optimized 3-dimensional in vivo predictive assay-based radiation therapy using positron emission tomography-computerized tomography imaging. Acta Oncol 2003;42:123-36.

8. Burgman P, Odonoghue JA, Humm JL, et al. Hypoxia-Induced increase in FDG uptake in MCF7 cells. J Nucl Med 2001;42:170-5.

9. Cerfolio RJ, Bryant AS, Winokur TS, et al. Repeat FDG-PET after neoadjuvant therapy is a predictor of pathologic response in patients with non-small cell lung cancer. Ann Thorac Surg 2004;78:1903-9.

10. Clavo AC, Brown RS, Wahl RL. Fluorodeoxyglucose uptake in human cancer cell lines is increased by hypoxia. J Nucl Med 1995;36:1625-32.

11. Cox JD, Azarnia N, Byhardt RW, et al. A randomized phase I/II trial of hyperfractionated radiation therapy with total doses of 60.0 Gy to 79.2 Gy: possible survival benefit with greater than or equal to $69.6 \mathrm{~Gy}$ in favorable patients with Radiation Therapy Oncology Group stage III non-small-cell lung carcinoma: report of Radiation Therapy Oncology Group 83-11. J Clin Oncol 1990;8:1543-55.

12. de Geus-Oei LF, Kaanders JH, Pop LA, et al. Effects of hyperoxygenation on FDG-uptake in head-and-neck cancer. Radiother Oncol 2006;80:51-60.

13. de Ruysscher D, Wanders S, Minken A, et al. Effects of radiotherapy planning with a dedicated combined PET-CTsimulator of patients with non-small cell lung cancer on dose limiting normal tissues and radiation dose-escalation: a planning study. Radiother Oncol 2005;77:5-10.

14. Dose Schwarz J, Bader M, Jenicke L, et al. Early prediction of response to chemotherapy in metastatic breast cancer using sequential 18F-FDG PET. J Nucl Med 2005;46:1144-50.

15. Downey RJ, Akhurst T, Gonen M, et al. Preoperative F-18 fluorodeoxyglucose-positron emission tomography maximal standardized uptake value predicts survival after lung cancer resection. J Clin Oncol 2004;22:3255-60.

16. Erdi YE, Macapinlac H, Rosenzweig KE, et al. Use of PET to monitor the response of lung cancer to radiation treatment. Eur J Nucl Med 2000;27:861-6.

17. Erdi YE, Rosenzweig K, Erdi AK, et al. Radiotherapy treatment planning for patients with non-small cell lung cancer using positron emission tomography (PET). Radiother Oncol 2002;62:51-60. 
18. Eschmann SM, Friedel G, Paulsen F, et al. Is standardized (18)F-FDG uptake value an outcome predictor in patients with stage III non-small cell lung cancer? Eur J Nucl Med Mol Imaging 2006;33:263-9.

19. Forshaw MJ, Gossage JA, Mason RC. Neoadjuvant chemotherapy for oesophageal cancer: the need for accurate response prediction and evaluation. Surgeon 2005;3:373-82.

20. Furuse K, Fukuoka M, Kawahara M, et al. Phase III study of concurrent versus sequential thoracic radiotherapy in combination with mitomycin, vindesine, and cisplatin in unresectable stage III non-small-cell lung cancer. J Clin Oncol 1999;17:2692-9.

21. Gagel B, Reinartz P, Demirel C, et al. [18F] fluoromisonidazole and [18F] fluorodeoxyglucose positron emission tomography in response evaluation after chemo-/radiotherapy of non-smallcell lung cancer: a feasibility study. BMC Cancer 2006;6:51.

22. Gallamini A, Rigacci L, Merli F, et al. The predictive value of positron emission tomography scanning performed after two courses of standard therapy on treatment outcome in advanced stage Hodgkin's disease. Haematologica 2006;91:475-81.

23. Geets X, Daisne JF, Tomsej M, et al. Impact of the type of imaging modality on target volumes delineation and dose distribution in pharyngo-laryngeal squamous cell carcinoma: comparison between pre- and per-treatment studies. Radiother Oncol 2006;78:291-7.

24. Grigsby PW, Siegel BA, Dehdashti F, et al. Posttherapy [18F] fluorodeoxyglucose ositron emission tomography in carcinoma of the cervix: response and outcome. $J$ Clin Oncol 2004;22:2167-71.

25. Higashi K, Ueda Y, Arisaka Y, et al. 18F-FDG uptake as a biologic prognostic factor for recurrence in patients with surgically resected non-small cell lung cancer. $J$ Nucl Med 2002;43:39-45.

26. Hoekstra CJ, Stroobants SG, Smit EF, et al. Prognostic relevance of response evaluation using [18F]-2-fluoro-2-deoxy-D-glucose positron emission tomography in patients with locally advanced non-small-cell lung cancer. J Clin Oncol 2005;23:8362-70.

27. ICRU. Prescribing, Recording and Reporting Photon Beam Therapy ICRU Report 50. 1993; ISBN 0-913394-48-3.

28. Johnston CJ, Williams JP, Elder A, et al. Inflammatory cell recruitment following thoracic irradiation. Exp Lung Res 2004;30:369-82.

29. Kong FM, Ten Haken R, Eisbruch A, et al. Non-small cell lung cancer therapy-related pulmonary toxicity: an update on radiation pneumonitis and fibrosis. Semin Oncol 2005;32:S42-54.

30. Kong FM, Ten Haken RK, Schipper MJ, et al. High-dose radiation improved local tumor control and overall survival in patients with inoperable/unresectable non-small cell lung cancer: long-term results of a radiation dose escalation study. Int J Radiat Oncol Biol Phys 2005;63:324-33.

31. Lin LL, Yang Z, Mutic S, et al. FDG-PET imaging for the assessment of physiologic volume response during radiotherapy in cervix cancer. Int J Radiat Oncol Biol Phys 2006;65:177-81.

32. Mac Manus MP, Hicks RJ, Matthews JP, et al. Positron emission tomography is superior to computed tomography scanning for response-assessment after radical radiotherapy or chemoradiotherapy in patients with non-small-cell lung cancer. J Clin Oncol 2003;21:128592.

33. Mac Manus MP, Hicks RJ, Matthews JP, et al. Metabolic (FDGPET) response after radical radiotherapy/chemoradiotherapy for non-small cell lung cancer correlates with patterns of failure. Lung Cancer 2005;49:95-108.

34. Martel MK, Ten Haken RK, Hazuka MB, et al. Estimation of tumor control probability model parameters from 3-D dose distributions of non-small cell lung cancer patients. Lung Cancer 1999;24:31-7. 
35. Miften M, Wiesmeyer M, Monthofer S, et al. Implementation of FFT convolution and multigrid superposition models in the FOCUS RTP system. Phys Med Biol 2000;45:817-33.

36. Minn H, Clavo AC, Wahl RL. Influence of hypoxia on tracer accumulation in squamouscell carcinoma: in vitro evaluation for PET imaging. Nucl Med Biol 1996;23:941-6.

37. Novello S, le Chevalier T. Is there a standard strategy in the management of locally advanced non-small cell lung cancer? Lung Cancer 2001;34:S9-S14.

38. Onishi H, Kuriyama K, Yamaguchi M, et al. Concurrent twodimensional radiotherapy and weekly docetaxel in the treatment of stage III non-small cell lung cancer: a good local response but no good survival due to radiation pneumonitis. Lung Cancer 2003;40:79-84.

39. Pottgen C, Levegrun S, Theegarten D, et al. Value of 18Ffluoro-2-deoxy-D-glucosepositron emission tomography/computed tomography in non-small-cell lung cancer for prediction of pathologic response and times to relapse after neoadjuvant chemoradiotherapy. Clin Cancer Res 2006;12:97-106.

40. Pugachev A, Ruan S, Carlin S, et al. Dependence of FDG uptake on tumor microenvironment. Int J Radiat Oncol Biol Phys 2005;62:545-53.

41. Rahmouni A, Luciani A, Itti E. MRI and PET in monitoring response in lymphoma. Cancer Imaging 2005;5 Spec. No. A:S106-12.

42. Rodrigues G, Lock M, D'Souza D, et al. Prediction of radiation pneumonitis by dosevolume histogram parameters in lung cancer - a systematic review. Radiother Oncol 2004;71:127-38.

43. Rube CE, Rodemann HP, Rube C. The relevance of cytokines in the radiation-induced lung reaction. Experimental basis and clinical significance. Strahlenther Onkol 2004;180:541-9.

44. Saunders M, Dische S, Barrett A, et al. Continuous, hyperfractionated, accelerated radiotherapy (CHART) versus conventional radiotherapy in non-small cell lung cancer: mature data from the randomised multicentre trial CHART Steering committee. Radiother Oncol 1999;52:137-48.

45. Sause W, Kolesar P, Taylor SI, et al. Final results of phase III trial in regionally advanced unresectable non-small cell lung cancer: Radiation Therapy Oncology Group, Eastern Cooperative Oncology Group, and Southwest Oncology Group. Chest 2000;117:358-64.

46. Seppenwoolde Y, Lebesque JV, de Jaeger K, et al. Comparing different NTCP models that predict the incidence of radiation pneumonitis Normal tissue complication probability. Int $J$ Radiat Oncol Biol Phys 2003;55:724-35.

47. Tanaka T, Furukawa $\mathrm{T}$, Fujieda $\mathrm{S}$, et al. Double-tracer autoradiography with $\mathrm{Cu}-$ ATSM/FDG and immunohistochemical interpretation in four different mouse implanted tumor models. Nucl Med Biol 2006;33:743-50.

48. Therasse P, Arbuck SG, Eisenhauer EA, et al. New guidelines to evaluate the response to treatment in solid tumors. European Organization for Research and Treatment of Cancer, National Cancer Institute of the United States, National Cancer Institute of Canada. $J$ Natl Cancer Inst 2000;92:205-16.

49. Thie JA. Understanding the standardized uptake value, its methods, and implications for usage. J Nucl Med 2004;45:1431-4.

50. van Baardwijk A, Baumert BG, Bosmans G, et al. The current status of FDG-PET in tumor volume definition in radiotherapy treatment planning. Cancer Treat Rev 2006;32:245-60.

51. Van der Meeren A, Monti P, Lebaron-Jacobs L, et al. Characterization of the acute inflammatory response after irradiation in mice and its regulation by interleukin 4 (I14). Radiat Res 2001;155:858-65.

52. van Der Wel A, Nijsten S, Hochstenbag M, et al. Increased therapeutic ratio by 18 FDG-PET CT planning in patients with clinical CT stage N2-N3M0 non-small-cell lung cancer: a modeling study. Int J Radiat Oncol Biol Phys 2005;61:649-55. 
53. Vansteenkiste JF, Stroobants SG, Dupont PJ, et al. Prognostic importance of the standardized uptake value on (18)F-fluoro-2-deoxy-glucose-positron emission tomography scan in nonsmall-cell lung cancer: an analysis of 125 cases. Leuven Lung Cancer Group. J Clin Oncol 1999;17:3201-6.

54. Vanuytsel LJ, Vansteenkiste JF, Stroobants SG, et al. The impact of (18)F-fluoro-2-deoxyD-glucose positron emission tomography (FDG-PET) lymph node staging on the radiation treatment volumes in patients with non-small cell lung cancer. Radiother Oncol 2000;55:317-24.

55. Vaupel P. The role of hypoxia-induced factors in tumor progression. Oncologist 2004;9:107.

56. Weber WA, Petersen V, Schmidt B, et al. Positron emission tomography in non-small-cell lung cancer: prediction of response to chemotherapy by quantitative assessment of glucose use. J Clin Oncol 2003;21:2651-7.

57. Xue F, Lin LL, Dehdashti F, et al. F-18 fluorodeoxyglucose uptake in primary cervical cancer as an indicator of prognosis after radiation therapy. Gynecol Oncol 2006;101:147-51.

58. Young H, Baum R, Cremerius U, et al. Measurement of clinical and subclinical tumor response using [18F]-fluorodeoxyglucose and positron emission tomography: review and 1999 EORTC recommendations. European Organization for Research and Treatment of Cancer (EORTC) PET Study Group. Eur J Cancer 1999;35:1773-82.

59. Zhang JZ, Behrooz A, Ismail-Beigi F. Regulation of glucose transport by hypoxia. Am J Kidney Dis 1999;34:189-202.

60. Zimny M, Gagel B, Dimartino E, et al. FDG-a marker of tumour hypoxia? A comparison with [(18)F]fluoromisonidazole and $\mathrm{pO}(2)$-polarography in metastatic head and neck cancer. Eur J Nucl Med Mol Imaging 2006;33:1426-31. 

CH A P T E R

Time trends in nodal volumes and motion during radiotherapy for patients with stage III non-small cell lung cancer

Int J Rad Oncol Biol Phys. 2007;Accepted for publication

Geert Bosmans Angela van Baardwijk

André Dekker

Michel Öllers

Stofferinus Wanders

Liesbeth Boersma

Philippe Lambin

Dirk De Ruysscher 


\begin{abstract}
Purpose: Knowledge of changes in GTV and motion of GTV during a course of radiotherapy is necessary for accurate treatment delivery. This study describes the time trends in nodal CT volume and nodal motion for patients with locally advanced NSCLC.

Patients and Methods: In a prospective clinical trial 12 patients, with a total of 22 positive nodes, underwent a CT-PET scan prior to treatment, as well as in the first and second week following start of radiotherapy. Volume changes could be measured for all nodes. For 21 nodes, the motion was measured based on a respiration correlated CT (RC-CT) scan. Repeated RC-CT scans were available for 11 nodes to evaluate the change in motion.

Results: In 6/22 (27\%) patients the nodal volume increased $>30 \%$ while in $3 / 22$ $(14 \%)$ the volume decreased $>30 \%$. On average, the nodal volume did not change significantly (from 4.9 to 5.1 to $4.6 \mathrm{~cm}^{3}$ ). The average motion of the nodal areas was initially $5.6 \mathrm{~mm} \pm 2.8 \mathrm{~mm}$. This motion decreased slightly during therapy but not statistically significant. However, large inter-patient and inter-nodal motion differences were observed.

Conclusion: A large variability of changes in nodal volume between patients was observed. However, this had limited clinical impact, since volumes and hence volume changes were small. The nodal motion did not change significantly during therapy. However, because of the large inter patient variability of nodal motion prior to treatment, internal margins for nodal areas should be calculated before radiotherapy using RC-CT, such that the margins can be applied for individual patients. Repeated imaging of the nodes seems however to be of limited use, as the observed individual changes in nodal volume and motion tend to fall within the commonly applied margins.
\end{abstract}




\section{INTRODUCTION}

Radiotherapy plays an important role in the management of patients suffering from non-small cell lung cancer (NSCLC). Obviously, accurate definition of target volumes is crucial. Several authors showed that the use of FDG-PET information improves target delineation, as indicated by a reduced interobserver variation This has been ascribed to the ability of FDG-PET to better discriminate involved from non-involved nodes, tumor from atelectasis and the application of automated contouring systems ${ }^{1-8}$.

Respiration correlated CT (RC-CT) scans have been shown to be able to take into account motion of the tumor ${ }^{9,10}$. With this relatively new technique tumor motion can be measured in 3 dimensions, and these data can subsequently be used to apply the internal margins, as defined by the ICRU ${ }^{11,12}$.

Besides optimal delineation before the start of radiation, adequate radiotherapy also needs to take into account the changes in volume and motion of target volumes during therapy. Indeed, many research groups have demonstrated striking volume changes during a course of radiotherapy ${ }^{13-17}$. In a previous paper we found that the motion of the primary tumor during a course of radiotherapy on average did not show significant changes, but for individual patients sometimes larger changes in motion were seen ${ }^{13}$. However, all studies presented data on the primary tumor, whereas none described changes of the volume of mediastinal lymph nodes. Even less data are available on the mobility of mediastinal lymph nodes prior to and during a course of radiation therapy. Moreover, only one study used four-dimensional CT scanning to assess nodal motion ${ }^{18}$ and other authors used surrogate methods to estimate nodal motion in three dimensions, like repeated CT imaging ${ }^{19}$ or orthogonal fluoroscopy of calcified lymph nodes ${ }^{20}$.

In summary, no data are available on changes of real time nodal motion and nodal volume during a course of radiotherapy. More detailed information on nodal motion and changes in nodal volume and motion would allow to individually adapt the internal margin and thereby allow further optimization of the treatment plans, i.e. to reduce geograhical misses whilst mimizing the dose to the normal tissues. Therefore, the aim of the present study was to analyze changes in nodal volumes and nodal motion during the first 2 weeks of an accelerated course of radiotherapy in patients with locally advanced non-small cell lung cancer (NSCLC), using RC-CT scans. 


\section{PATIENTS AND METHODS}

\section{Patients}

Twenty-three patients with histological or cytological proven non-small cell lung cancer (NSCLC) were enrolled in this prospective study, which was part of a phase I trial. Patients were included from December 2004 until September 2005. Five patients had initially no positive lymph nodes (N0), while four patients became node negative on the FDG-PET scan after chemotherapy. Two patients had a primary tumor with direct extensive invasion in the mediastinum, making distinguishing the primary tumor and the involved lymph nodes impossible. This resulted in a total of 12 patients with 22 positive nodal areas. The Naruke/ATS-LCSG standardized mapping system was used to define the anatomical location of the lymph node involvement ${ }^{21}$. No concurrent chemotherapy was given.

The Medical Ethics Committee according to the Dutch law approved this study. All patients gave written informed consent before entering this study.

\section{Radiotherapy simulation}

Patients were simulated in radiotherapy position on a dedicated CT-PETsimulator with both arms above the head using an immobilization device and patient laser marker system. Radiotherapy technologists trained specifically for this purpose carried out the simulation. The CT-PET scanner used was a Siemens Biograph (SOMATOM Sensation-16 with an ECAT ACCEL PET scanner). A spiral CT with intravenous contrast was performed covering the complete thoracic region ${ }^{13}$.

\section{Respiration Correlated CT scans (RC-CT)}

The CT-PET was immediately followed by a respiration correlated CT, using offline correlation of the 16-slice spiral CT scan of the CT-PET system. This had been modified to enable a low pitch (minimum 0.1), in combination with a respiratory signal obtained by a pressure sensor in a chest belt (AZ-733V, Anzai Medical Corporation, Tokyo, Japan). The raw CT data were reconstructed in 10 phases, from $0 \%$ to $100 \%$ in steps of $10 \%$ of the respiration period starting at end inspiration. In-house developed software with Matlab (Mathworks Inc, Natick, MA) was used to measure the motion of the lymph node in all three orthogonal directions ${ }^{9}$. This was performed manually by measuring the largest distance between the respiration phases on a movie in an axial, sagittal and coronal view. 


\section{Radiotherapy planning}

Radiotherapy planning was performed on an $\mathrm{XiO}$ (Computerized Medical Systems, St Louis, Missouri) treatment planning system, using inhomogeneity corrections, based on a convolution algorithm. For all patients, the Gross Tumor Volume (GTV) was defined based on CT-PET data, obtained prior to radiotherapy (i.e. post chemotherapy in case of induction chemotherapy for the primary tumor and pre chemotherapy for the lymph nodes). For the volume comparison in this study, the CT-Nodal Volume was defined as the FDG-PET positive lymph nodes, as described by the report of nuclear medicine postchemotherapy, delineated on the $\mathrm{CT}$ with a mediastinal window-level setting $(\mathrm{W}=600, \mathrm{~L}=40)$.

\section{Radiotherapy dose and normal tissue dose constraints}

For each patient, the radiation dose was escalated to a mean lung dose (MLD) of $19 \mathrm{~Gy} \pm 1 \mathrm{~Gy}$ while respecting a maximum spinal cord dose of $54 \mathrm{~Gy}$. There were no esophageal dose constraints. The maximal allowed total tumor dose (TTD) was 79.2 Gy or lower if any of the normal tissue dose constraints were reached. Radiotherapy was always delivered twice a day (BID), with a minimum of 8 hours interval between the two fractions, and given 5 days per week with a fraction dose of $1.8 \mathrm{~Gy}$.

\section{Study design}

Patients underwent repeated CT-PET scans and respiration correlated CT scans in treatment position, before and one and two weeks during radiotherapy. At all time points, the nodal volume was delineated using the free-breathing CT. Although in principle this may have led to blurring artifacts, our high-speed 16 slice CT-scan protocol effectively "freezes" the lesion in a single phase during a free breathing CT-scan. This assumption was found to be valid for the primary tumor and was subsequently extrapolated to the nodal areas. The nodal volume was delineated for each patient by the same physician to avoid interobserver variability. To study the intra-observer variability, seven (32\%) nodal volumes were delineated twice by the same physician for all time points, with at least a 3 months time interval. The ratio between the two delineations was calculated and reported as a percentage.

Due to technical and logistical problems, a complete image dataset at all three time points, was not available for two patients. A CT-PET dataset (with in total four nodal areas) two weeks after the start of treatment was not obtained for these two patients. 


\section{Endpoints and statistical analysis}

We determined the nodal volumes (NV) prior to radiation, 1 and 2 weeks after start of the treatment, based on the NVs drawn on the free-breathing CT. In addition, the respiration correlated $\mathrm{CT}$ was used to quantify the nodal motion at all 3 time points. Nodal motion was expressed as a $3 \mathrm{D}$ vector ${ }^{22}$, which is the quadratic sum of the motions in the three orthogonal directions. All results are expressed as the mean \pm standard deviation (SD). Statistical differences between paired parameters from the three time points were evaluated using the Wilcoxon signed rank test. Differences were considered to be significant when the p-value was less than 0.05 .

\begin{tabular}{|c|c|c|c|c|c|c|c|}
\hline $\begin{array}{l}\text { Patient } \\
\text { no. }\end{array}$ & $\begin{array}{c}\text { Age } \\
\text { (years) }\end{array}$ & Gender & $\begin{array}{l}\text { Clinical } \\
\text { stage }\end{array}$ & $\begin{array}{l}\text { Involved nodal } \\
\text { stations }\end{array}$ & $\begin{array}{l}\text { TTD } \\
\text { (Gy) }\end{array}$ & $\begin{array}{l}\text { MLD } \\
\text { (Gy) }\end{array}$ & $\begin{array}{l}\text { OTT } \\
\text { (days) }\end{array}$ \\
\hline 1 & 54 & M & T3N2M0 & 7 & 54.0 & 19.3 & 20 \\
\hline 2 & 75 & M & T3N2M0 & $7,4 \mathrm{R}$ & 54.0 & 15.2 & 20 \\
\hline 3 & 71 & M & $\mathrm{T} 2 \mathrm{~N} 3 \mathrm{M} 0$ & $7,3,5,2 \mathrm{R}$ & 54.0 & 14.7 & 23 \\
\hline 4 & 61 & M & T1N2M0 & $7,4 \mathrm{R}$ & 68.4 & 14.9 & 22 \\
\hline 5 & 77 & M & T4N2M0 & $7,4 \mathrm{R}, 10 \mathrm{R}$ & 61.2 & 17.6 & 22 \\
\hline 6 & 57 & M & T1N2M0 & $2 \mathrm{R}, 4 \mathrm{R}$ & 64.8 & 8.5 & 27 \\
\hline 7 & 59 & $\mathrm{~F}$ & $\mathrm{~T} 2 \mathrm{~N} 2 \mathrm{M} 0$ & $7,2 \mathrm{R}$ & 46.8 & 20.0 & 16 \\
\hline 8 & 55 & M & T4N2M0 & 5 & 79.2 & 16.4 & 29 \\
\hline 9 & 52 & $\mathrm{~F}$ & $\mathrm{~T} 2 \mathrm{~N} 2 \mathrm{M} 0$ & $7,10 \mathrm{~L}$ & 54.0 & 15.6 & 20 \\
\hline 10 & 50 & $\mathrm{~F}$ & T4N2M0 & 7 & 61.2 & 20.8 & 21 \\
\hline 11 & 71 & M & T4N2M0 & $2,4 \mathrm{R}$ & 79.2 & 13.6 & 29 \\
\hline 12 & 54 & M & $\mathrm{T} 2 \mathrm{~N} 3 \mathrm{M} 0$ & $2 \mathrm{~L}$ & 79.2 & 16.1 & 30 \\
\hline Mean & 61.3 & & & & 63.0 & 16.1 & 23.3 \\
\hline SD & 9.6 & & & & 11.3 & 3.3 & 4.5 \\
\hline
\end{tabular}

\section{RESULTS}

\section{Patient characteristics}

Patient characteristics are shown in Table 1. The mean values of the total tumor dose (TTD) and the corresponding mean lung dose (MLD) were $63.0 \mathrm{~Gy} \pm 11.3$ Gy [range: $46.8 \mathrm{~Gy}-79.2 \mathrm{~Gy}$ ] and 16.1 Gy $\pm 3.3 \mathrm{~Gy}$ [range: $8.5 \mathrm{~Gy}-20.8 \mathrm{~Gy}$ ], respectively. The average volume per pathological lymph node before radio- 
therapy was $4.9 \mathrm{~cm}^{3} \pm 4.4 \mathrm{~cm}^{3}$ [range: $0.2 \mathrm{~cm}^{3}-19.6 \mathrm{~cm}^{3}$ ]. The first repeat scan was made after the patient received on average a dose of 21.2 Gy \pm 4.4 Gy (about 12 fractions) and the second scan after 38.5 Gy \pm 5.2 Gy (about 21 fractions).

\section{Volume changes of the mediastinal lymph nodes}

A large heterogeneity of nodal volume changes between patients was observed at all time points (Table 2). In 6/22 (27\%) patients the nodal volume increased $>$ $30 \%$, while in $3 / 22(14 \%)$ the volume decreased $>30 \%$. For the majority of nodal areas (13/22) the volume changed only slightly $(<30 \%)$ (Fig. 1).

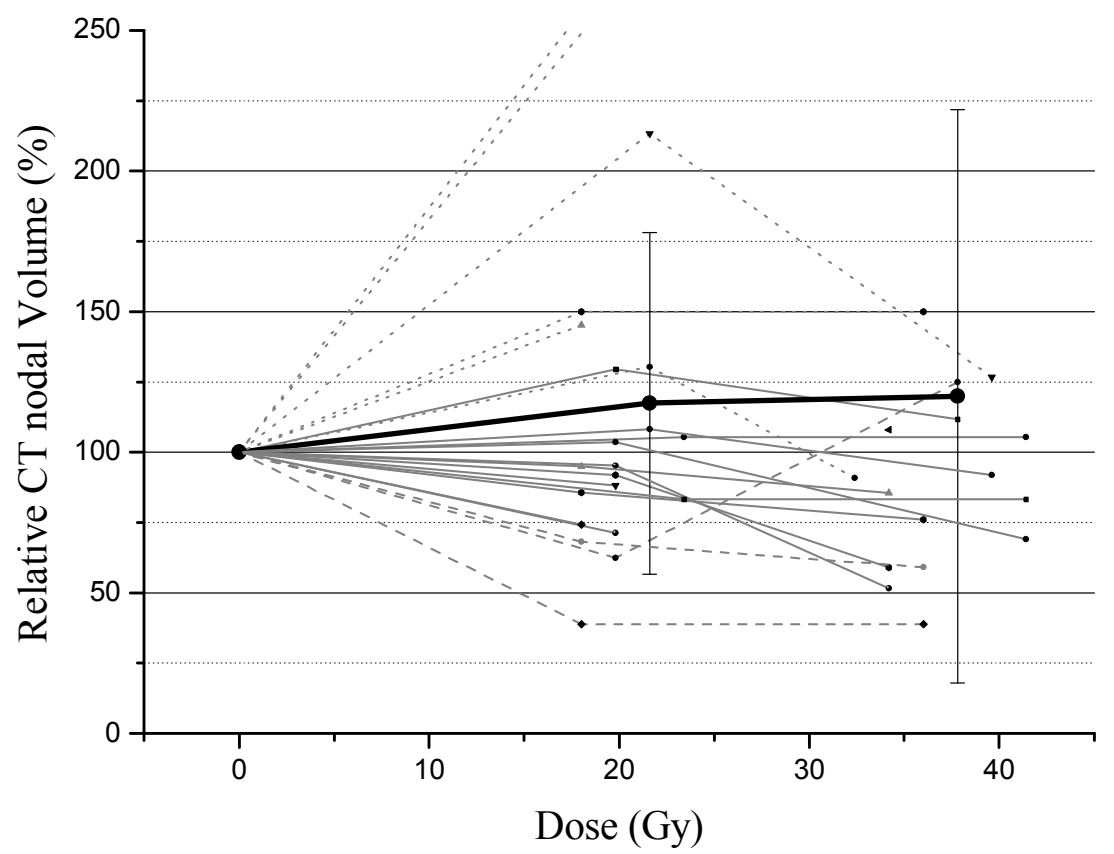

Figure 1: Nodal volumes (NV), relative to the volume prior to treatment as a function of the dose. The thick black line represents the mean relative CT volume together with the standard deviation. The dotted lines represent the individual patients where the NV increased more than $30 \%$; the dashed lines those patients with a decrease of more than $30 \%$. A solid grey line is used for the remaining patients.

On average the nodal volume changed from $4.9 \mathrm{~cm}^{3} \pm 4.4 \mathrm{~cm}^{3}$ prior to treatment, to $5.1 \mathrm{~cm}^{3} \pm 4.1 \mathrm{~cm}^{3}$ and $4.6 \mathrm{~cm}^{3} \pm 4.2 \mathrm{~cm}^{3} 1$ and 2 weeks after the start of treatment, respectively. These changes were not significant $(\mathrm{p}=0.897$ prior versus week $1, \mathrm{p}=0.177$ prior versus week 2 and $\mathrm{p}=0.084$ week 2 versus week 1 ). 


\section{Intra-observer variability in delineation of the mediastinal lymph nodes}

For the 21 delineations on three different time points which were performed twice an average intra-observer variability of $11.6 \% \pm 9.4 \%$, with a maximum deviation of $37.4 \%$ was found. Since similar volume changes were observed for the second delineation (data not shown) this variability did not influence the results on the volume changes of the mediastinal areas during radiotherapy.

\begin{tabular}{|c|c|c|c|c|}
\hline $\begin{array}{l}\text { Patient } \\
\text { no. }\end{array}$ & $\begin{array}{c}\text { Nodal } \\
\text { area }\end{array}$ & $\begin{array}{l}\text { Volume be- } \\
\text { fore RT } \\
\left(\mathrm{cm}^{3}\right)\end{array}$ & $\begin{array}{c}\text { Volume one week after } \\
\text { start RT }\left(\mathrm{cm}^{3}\right) \text { and relative } \\
\text { change }(\%)\end{array}$ & $\begin{array}{c}\text { Volume two weeks after } \\
\text { start RT }\left(\mathrm{cm}^{3}\right) \text { and relative } \\
\text { change }(\%)\end{array}$ \\
\hline 1 & 7 & 11.8 & $11.2(-5 \%)$ & $10.1(-14 \%)$ \\
\hline \multirow[t]{2}{*}{2} & 7 & 3.5 & $2.6(-26 \%)$ & NA \\
\hline & $4 \mathrm{R}$ & 3.1 & $4.5(+45 \%)$ & NA \\
\hline \multirow[t]{4}{*}{3} & 7 & 2.2 & $1.5(-32 \%)$ & $1.3(-41 \%)$ \\
\hline & $2 \mathrm{R}$ & 19.6 & $16.8(-14 \%)$ & $14.9(-24 \%)$ \\
\hline & 3 & 0.2 & $0.3(+50 \%)$ & $0.3(+50 \%)$ \\
\hline & 5 & 1.8 & $0.7(-61 \%)$ & $0.7(-61 \%)$ \\
\hline \multirow[t]{2}{*}{4} & 7 & 6.2 & $5.9(-5 \%)$ & $3.2(-48 \%)$ \\
\hline & $4 \mathrm{R}$ & 6.1 & $5.6(-8 \%)$ & $3.6(-41 \%)$ \\
\hline \multirow[t]{3}{*}{5} & $10 \mathrm{R}$ & 3.6 & $9.5(+163 \%)$ & $10.9(+202 \%)$ \\
\hline & $4 \mathrm{R}$ & 2.9 & $3.0(+3 \%)$ & $2.0(-31 \%)$ \\
\hline & 7 & 1.8 & $4.9(+172 \%)$ & $8.2(+355 \%)$ \\
\hline \multirow[t]{2}{*}{6} & $2 \mathrm{R}$ & 9.3 & $8.2(-12 \%)$ & NA \\
\hline & $4 \mathrm{R}$ & 6.6 & $4.7(-29 \%)$ & NA \\
\hline \multirow[t]{2}{*}{7} & $2 \mathrm{R}$ & 0.6 & $0.5(-17 \%)$ & $0.5(-17 \%)$ \\
\hline & 7 & 5.7 & $6.0(+5)$ & $6.0(+5 \%)$ \\
\hline 8 & 5 & 0.8 & $0.5(-38 \%)$ & $1.0(+25 \%)$ \\
\hline \multirow[t]{2}{*}{9} & $10 \mathrm{~L}$ & 1.5 & $3.2(+113 \%)$ & $1.9(+27 \%)$ \\
\hline & 7 & 6.1 & $6.6(+8 \%)$ & $5.6(-8 \%)$ \\
\hline 10 & 7 & 3.4 & $4.4(+29 \%)$ & $3.8(+12 \%)$ \\
\hline 11 & $2,4 \mathrm{R}$ & 7.6 & $9.9(+30 \%)$ & $6.9(-9 \%)$ \\
\hline 12 & $2 \mathrm{~L}$ & 2.5 & $2.7(+8 \%)$ & $1.9(-24 \%)$ \\
\hline Mean & & 4.9 & $5.1(+4 \%)$ & $4.6(-6 \%)$ \\
\hline SD & & 4.4 & 4.1 & 4.2 \\
\hline
\end{tabular}




\section{Motion changes of nodal areas}

Nodal motion is shown in Table 3 and Fig 2. For one nodal area it was not possible to measure the motion because of its limited size $\left(0.2 \mathrm{~cm}^{3}\right)$. The mean initial motion before start of radiotherapy of all nodes was $5.6 \mathrm{~mm} \pm 2.8 \mathrm{~mm}$. The motion was larger in cranial-caudal direction $(4.3 \mathrm{~mm} \pm 3.2 \mathrm{~mm})$ compared with the other directions (ventro-dorsal $1.8 \mathrm{~mm} \pm 1.5 \mathrm{~mm}$ and left-right $2.0 \mathrm{~mm}$ $\pm 1.3 \mathrm{~mm}$ ). The 3D motion changed to $5.8 \mathrm{~mm} \pm 2.6 \mathrm{~mm}$ and $5.3 \mathrm{~mm} \pm 2.7 \mathrm{~mm}$ one and two weeks after the start of the radiotherapy respectively. All differences compared to the initial nodal motion were not statistically significant different from each other $(p=0.838$ and $p=0.221$ respectively).

\begin{tabular}{|c|c|c|c|c|}
\hline $\begin{array}{l}\text { Patient } \\
\text { no. }\end{array}$ & $\begin{array}{l}\text { Nodal } \\
\text { area }\end{array}$ & $\begin{array}{l}\text { Motion prior to } \\
\text { treatment }(\mathrm{mm})\end{array}$ & $\begin{array}{c}\text { Motion after one } \\
\text { week of RT (mm) }\end{array}$ & $\begin{array}{c}\text { Motion after two } \\
\text { weeks of RT (mm) }\end{array}$ \\
\hline 1 & 7 & 4.9 & & \\
\hline \multirow[t]{2}{*}{2} & 7 & 3.6 & & \\
\hline & $4 R$ & 6.4 & & \\
\hline \multirow[t]{4}{*}{3} & 7 & 4.2 & 4.0 & 1.4 \\
\hline & $2 \mathrm{R}$ & 2.1 & 2.2 & 2.2 \\
\hline & 3 & NA & & \\
\hline & 5 & 1.4 & 3.7 & 1.4 \\
\hline \multirow[t]{2}{*}{4} & 7 & 6.7 & 9.3 & 6.1 \\
\hline & $4 \mathrm{R}$ & 6.7 & 7.8 & 7.2 \\
\hline \multirow[t]{3}{*}{5} & $10 \mathrm{R}$ & 3.3 & & \\
\hline & $4 R$ & 3.7 & & \\
\hline & 7 & 4.3 & & \\
\hline \multirow[t]{2}{*}{6} & $2 \mathrm{R}$ & 6.4 & & \\
\hline & $4 \mathrm{R}$ & 7.0 & & \\
\hline \multirow[t]{2}{*}{7} & $2 \mathrm{R}$ & 12.3 & 9.2 & 7.9 \\
\hline & 7 & 6.7 & 6.3 & 9.0 \\
\hline 8 & 5 & 4.3 & 4.6 & 3.3 \\
\hline \multirow[t]{2}{*}{9} & $10 \mathrm{~L}$ & 9.1 & 9.1 & 6.7 \\
\hline & 7 & 3.9 & 3.6 & 6.7 \\
\hline 10 & 7 & 11.0 & 4.4 & 6.3 \\
\hline 11 & $2,4 \mathrm{R}$ & 7.7 & & \\
\hline 12 & $2 \mathrm{~L}$ & 2.1 & & \\
\hline Mean & & 5.6 & 5.8 & 5.3 \\
\hline SD & & 2.8 & 2.6 & 2.7 \\
\hline
\end{tabular}


In order to investigate the influence of the anatomical location of the nodes on its motion, the involved lymph nodes were classified based on their location: infra-carinal $(\mathrm{N}=10)$ versus supra-carinal $(\mathrm{N}=12)$. The motion was $5.8 \mathrm{~mm} \pm$ $2.6 \mathrm{~mm}$ versus $5.5 \mathrm{~mm} \pm 3.2 \mathrm{~mm}$ respectively $(\mathrm{p}=0.812)$. However, in individual patients, large inter-nodal motion differences could occur (e.g. patient nrs: 2 , 7 and 9) (Table 3).

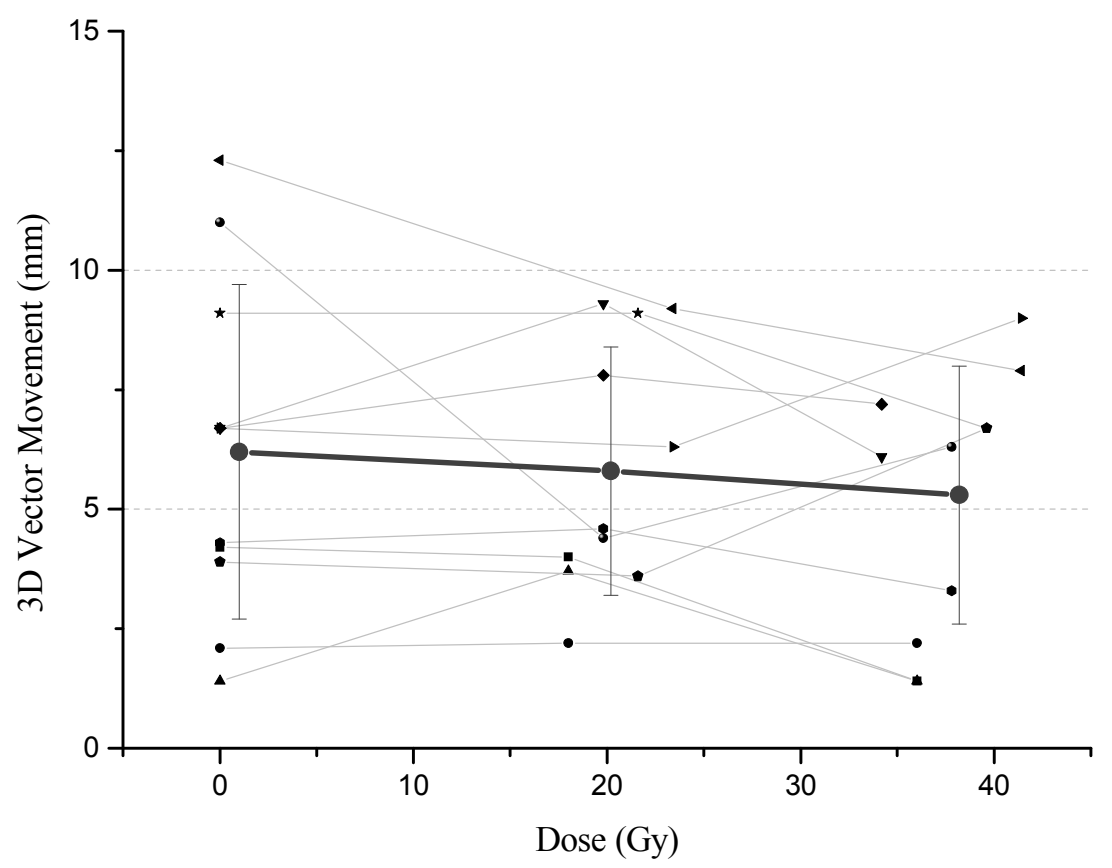

Figure 2: 3D Vector motion (quadratic sum of the motion in three orthogonal directions) as a function of the dose. The gray lines represent the individual patient data, while the thick black line represents the mean tumor motion, together with the standard deviation.

\section{DISCUSSION}

To the best of our knowledge, this is the first report about time trends of nodal volume and motion of involved mediastinal lymph nodes in patients with NSCLC during radiotherapy. This information is clinically relevant since changes in size and mobility of all tumor localizations (e.g. the primary tumor and the lymph nodes) during a course of radiotherapy are needed as an input for adaptive radiotherapy approaches. 
Our results show that, similar to the volume changes of the primary tumor volumes ${ }^{13}$, the individual volumes of the mediastinal lymph nodes may change considerably during radiotherapy and that a large heterogeneity of nodal volume changes between patients was observed. The nodal volume increased $>30 \%$ in $27 \%$ of the patients and decreased $>30 \%$ in $14 \%$ of the patients. In a previous study of our group, for the primary tumor, $17 \%$ of the patients showed a large increase of tumor volume and $13 \%$ of the patients had a large decrease of the tumor $^{13}$. We did not find a correlation between the tumor increase and decrease versus the change in volume of the involved mediastinal lymph nodes in the same patient. Intra-patient differences were also observed that may be explained by the different location and the size of the nodal areas within the patient.

Intra-observer variability could not explain the time trends of the nodal volume changes. Remarkably, the relative intra-observer variability for delineation of the nodes was substantially larger than the intra-observer variability of the primary tumor $(1.2 \% \pm 4.4 \%){ }^{13}$. This can probably be ascribed to the fact that a small intra-observer variation in the small absolute nodal volumes has larger relative effect than small intra-observer variation in the large primary tumor volumes.

However, since the nodal volume was relatively small (average $4.9 \mathrm{~cm}^{3}$, range: $0.2 \mathrm{~cm}^{3}-19.6 \mathrm{~cm}^{3}$ ), the clinical impact of the volume changes is probably limited. Assuming that nodes are spherical, the average observed volume change would result in a difference of the radius of $1 \mathrm{~mm}$ or less for $85 \%$ of the investigated nodes [range: $-2 \mathrm{~mm}-+5 \mathrm{~mm}$ ]. Only in one patient two lymph nodes showed an increase of respectively $4 \mathrm{~mm}$ and $5 \mathrm{~mm}$ of the radius. In this case, adaptation of the gross tumor volume might have been appropriate depending on the used treatment technique (e.g. motion adapted) and margins. The motion of those lymph nodes was relatively small (3D vector: $3.3 \mathrm{~mm}$ and $4.3 \mathrm{~mm}$ respectively). Our clinically applied GTV-PTV margin of $1 \mathrm{~cm}$, consisting of a 3 $\mathrm{mm}$ margin for CTV ${ }^{23}$ and $7 \mathrm{~mm}$ for PTV, would probably have covered most of the change in volume during therapy. This, in contrast with the changes of the volume of the primary tumor during therapy, where in some patients significant changes were observed and adaptive radiotherapy could be beneficial ${ }^{13}$.

The motion of the nodal areas, which are more constrained by surrounding tissue, was smaller than the motion of the primary tumor. Prior to therapy, the $3 \mathrm{D}$ vector was on average $5.6 \mathrm{~mm} \pm 2.8 \mathrm{~mm}$ versus $7.8 \mathrm{~mm} \pm 4.8 \mathrm{~mm}{ }^{13}$ for the nodes and primary tumor respectively. These results are in agreement with Piet et al. ${ }^{18}$, who also used 4D-CT scans to evaluate the nodal motion. They reported a $3 \mathrm{D}$ vector motion of $6.2 \mathrm{~mm} \pm 2.9 \mathrm{~mm}$. Jenkins et al. ${ }^{20}$ used orthogonal fluoroscopic imaging to asses the motion of calcified lymph nodes. These investigators observed the motion during several breathing cycles and therefore have probably taken the maximum respiration excursion. They found a larger nodal motion of $7.0 \mathrm{~mm} \pm 4.7 \mathrm{~mm}$ especially in the cranial caudal direction. 
The difference with our results may be due to the motion measurement method and to a different patient population.

Taking into account the peak-to-peak amplitude divided by four in our study to calculate the internal margin ${ }^{9}$, an average isotropic internal margin of $1.4 \mathrm{~mm}$ would suffice, so the $5 \mathrm{~mm}$ margin proposed by van Sörnsen de Koste et al. ${ }^{19}$, seems rather large. However, since these authors used repeated 3D CT images, other factors like image correlation and intra-observer variations may have influenced their results. In the present study, we found a large inter-patient and inter-nodal difference in motion (range: $1.4 \mathrm{~mm}-12.3 \mathrm{~m}$ ), indicating that a general internal margin is not appropriate and the motion should individually be measured with an RC-CT. This might improve target definition and hence treatment outcome. In agreement with the other investigators ${ }^{18-20}$, we did not observe a difference in motion between the locations of the nodal areas.

On average no change in nodal motion was observed during treatment although for some patients large differences could occur. When using a specific nodal internal margin these motion changes could influence treatment accuracy and adaptive strategies might be developed if the changes extend beyond the applied PTV margins.

Until now respiratory management is mainly focused on the motion of the primary tumor ${ }^{10}$. When extending this high precision treatment technique to the involved lymph nodes, the reported nodal volume and motion changes could play an important role in the accuracy of the treatment.

In conclusion, our results show that a different internal margin should be applied to the nodal areas compared to the primary tumor. This internal margin should be defined individually with a four-dimensional imaging technique prior to therapy. On average the nodal volume as well as the nodal motion did not change significantly during the radiation although large inter patient differences were observed. In contrast to the primary tumor ${ }^{13}$, repeated imaging of the nodes seems to be of limited use, as the observed individual changes in nodal volume and motion tend to fall within the applied margins.

\section{REFERENCES}

1. De Ruysscher D, Wanders S, van Haren E, et al. Selective mediastinal node irradiation based on FDG-PET scan data in patients with non-small-cell lung cancer: a prospective clinical study. Int J Radiat Oncol Biol Phys 2005;62:988-994.

2. Erdi YE, Rosenzweig K, Erdi AK, et al. Radiotherapy treatment planning for patients with non-small cell lung cancer using positron emission tomography (PET). Radiother Oncol 2002;62:51-60.

3. Mah K, Caldwell CB, Ung YC, et al. The impact of (18)FDG-PET on target and critical organs in CT-based treatment planning of patients with poorly defined non-small-cell lung carcinoma: a prospective study. Int J Radiat Oncol Biol Phys 2002;52:339-350. 
4. Senan S, De Ruysscher D. Critical review of PET-CT for radiotherapy planning in lung cancer. Crit Rev Oncol Hematol 2005;56:345-351.

5. Senan S, De Ruysscher D, Giraud P, et al. Literature-based recommendations for treatment planning and execution in high-dose radiotherapy for lung cancer. Radiother Oncol 2004;71:139-146.

6. Steenbakkers RJ, Duppen JC, Fitton I, et al. Reduction of observer variation using matched CT-PET for lung cancer delineation: a three-dimensional analysis. Int J Radiat Oncol Biol Phys 2006;64:435-448.

7. van Baardwijk A, Bosmans G, Boersma L, et al. PET-CT-Based Auto-Contouring in NonSmall-Cell Lung Cancer Correlates With Pathology and Reduces Interobserver Variability in the Delineation of the Primary Tumor and Involved Nodal Volumes. Int J Radiat Oncol Biol Phys 2007;68:771-778.

8. van Der Wel A, Nijsten S, Hochstenbag M, et al. Increased therapeutic ratio by 18FDG-PET CT planning in patients with clinical CT stage N2-N3M0 non-small-cell lung cancer: a modeling study. Int J Radiat Oncol Biol Phys 2005;61:649-655.

9. Bosmans G, Buijsen J, Dekker A, et al. An "in silico" clinical trial comparing free breathing, slow and respiration correlated computed tomography in lung cancer patients. Radiother Oncol 2006;81:73-80.

10. Keall PJ, Mageras GS, Balter JM, et al. The management of respiratory motion in radiation oncology report of AAPM Task Group 76. Med Phys 2006;33:3874-3900.

11. ICRU report 50: Prescribing, recording, and reporting photon beam therapy. Bethesda, MD: International Commission on Radiation Units and Measurements; 1993.

12. ICRU report 62: Prescribing, recording, and reporting photon beam therapy (Supplement to ICRU report 50). Bethesda, MD: International Commission on Radiation Units and Measurements; 1999.

13. Bosmans G, van Baardwijk A, Dekker A, et al. Intra-patient variability of tumor volume and tumor motion during conventionally fractionated radiotherapy for locally advanced nonsmall-cell lung cancer: a prospective clinical study. Int J Radiat Oncol Biol Phys 2006;66:748-753.

14. Britton KR, Starkschall G, Tucker SL, et al. Assessment of gross tumor volume regression and motion changes during radiotherapy for non-small-cell lung cancer as measured by four-dimensional computed tomography. Int J Radiat Oncol Biol Phys 2007.

15. Kupelian PA, Ramsey C, Meeks SL, et al. Serial megavoltage CT imaging during external beam radiotherapy for non-small-cell lung cancer: observations on tumor regression during treatment. Int J Radiat Oncol Biol Phys 2005;63:1024-1028.

16. Siker ML, Tome WA, Mehta MP. Tumor volume changes on serial imaging with megavoltage CT for non-small-cell lung cancer during intensity-modulated radiotherapy: how reliable, consistent, and meaningful is the effect? Int J Radiat Oncol Biol Phys 2006;66:135141.

17. Underberg RW, Lagerwaard FJ, van Tinteren H, et al. Time trends in target volumes for stage I non-small-cell lung cancer after stereotactic radiotherapy. Int J Radiat Oncol Biol Phys 2006;64:1221-1228.

18. Piet AH, Lagerwaard FJ, Kunst PW, et al. Can mediastinal nodal mobility explain the low yield rates for transbronchial needle aspiration without real-time imaging? Chest 2007;131:1783-1787.

19. van Sornsen de Koste JR, Lagerwaard FJ, Nijssen-Visser MR, et al. What margins are necessary for incorporating mediastinal nodal mobility into involved-field radiotherapy for lung cancer? Int J Radiat Oncol Biol Phys 2002;53:1211-1215.

20. Jenkins P, Salmon C, Mannion C. Analysis of the movement of calcified lymph nodes during breathing. Int J Radiat Oncol Biol Phys 2005;61:329-334. 
21. Mountain CF, Dresler CM. Regional lymph node classification for lung cancer staging. Chest 1997;111:1718-1723.

22. van Sornsen de Koste JR, Lagerwaard FJ, Nijssen-Visser MR, et al. Tumor location cannot predict the mobility of lung tumors: a 3D analysis of data generated from multiple CT scans. Int J Radiat Oncol Biol Phys 2003;56:348-354.

23. Yuan S, Meng X, Yu J, et al. Determining optimal clinical target volume margins on the basis of microscopic extracapsular extension of metastatic nodes in patients with non-smallcell lung cancer. Int J Radiat Oncol Biol Phys 2007;67:727-734. 
C H A P T E R

General Discussion and Future Perspectives 

For stage III non-small cell lung cancer (NSCLC) patients, radiation therapy plays a major role in their treatment with curative intent. Nevertheless, the prognosis remains grim, with most patients still experiencing local and distant tumor relapse.

Local tumor control and hence survival could be improved by delivering higher doses to the tumor in a reasonably short overall treatment time, while at the same time keeping the radiation dose to surrounding healthy tissue tolerable.

Problems to achieve this in clinical practice are respiration artifacts in imaging of the tumor and changes in the tumor during radiation therapy.

Accurate imaging of lung tumors is difficult since respiration causes distorted images. Four-dimensional imaging, with the time being the fourth dimension, is therefore currently a 'hot topic' in radiotherapy. This technique is needed to achieve the accuracy of state-of-the art treatment delivery.

Radiation treatment planning is mostly based on a single pre-treatment CT(PET) scan, but incorporating the tumor dynamics during a course of radiotherapy into the treatment planning could probably improve treatment accuracy.

\section{Respiration Correlated (RC) or Four-Dimensional (4D) CT}

We first investigated how to use the 4D imaging data in the treatment planning. We compared the standard imaging protocol, a free breathing CT, with different methods of generating an internal target volume (ITV) based on the 4D or respiration correlated CT scan. With a free breathing CT, no information about the respiration motion is known. Therefore general population based margins are used to create the planning target volume (PTV). These margins will be too big for some patients, where the tumor will not move that much, but in other patients, with a large tumor motion, the margin will be too small. For the first group, knowledge of the tumor motion in the individual patient could lead to a decrease in treatment fields and hence sparing more normal tissue, resulting in a lower normal tissue complication probability (NTCP). Another possibility would be to keep the same level of toxicity and to escalate the radiation dose to reach these normal tissue dose constraints. For the second group, the treatment fields should be enlarged, thereby reducing geographical misses and increasing the tumor control probability (TCP).

Besides inappropriate margins, free breathing CT also might introduce systematic errors. The free breathing CT, with current fast multislice CT scanners, is actually a snapshot of the patient's anatomy and could be taken at an extreme position near end expiration or end inspiration of the tumor. As such a CT does not represent patient geometry during irradiation, a large systematic error may be introduced, which will have a big impact on the treatment accuracy ${ }^{1}$.

One might overcome this problem by acquiring a slow CT instead of the snapshot, free breathing CT. We therefore simulated a slow CT by averaging all the 
CTs taken at individual phases, and showed that it was sometimes difficult to delineate the tumor because of the blurred images, especially near the mediastinum or diaphragm. This resulted in too small volumes (Chapter 2) and therefore no optimal coverage of the Clinical Target Volume could be achieved.

Treatment plans based on all the CT datasets (i.e. the full 4D-CT) will have better CTV coverage but this method generated larger volumes than the treatment plan based on the half-ventilation CT scan.

The half-ventilation method seems to be optimal since only one CT has to been delineated, individual anisotropic tumor motion can be incorporated and the proposed margin recipe of the peak-to-peak amplitude divided by four still achieved good CTV coverage (Chapter 2).

The main advantage of using 4D-CT in treatment planning is reducing systematic errors, with the half-ventilation phase, and for some patients decreasing the internal safety margins which make dose escalation possible.

\section{Respiration Correlated CT-PET}

CT-PET imaging has proven to be beneficial for radiation treatment planning. It provides a more accurate estimate of the true extent of disease inside the thorax compared to CT and is a sensitive method for detection of distant metastasis outside the thorax ${ }^{2}$. PET can also better identify involved lymph nodes than CT and it has been proven to be safe to only irradiate PET positive lymph nodes ${ }^{3}$.

However, it is difficult to define the GTV manually on a PET scan, since these images are for instance very susceptible to window level settings. Many suggestions have been made for automatic delineations ${ }^{4}$, but no good threedimensional validation with pathologic information has been performed. One of the prerequisites of auto-delineation is a quantitative PET, which is a challenge in a tumor moving with respiration. The main problem is the smearing out effect due to the respiration, which makes the intensity of a PET voxel not only a function of glucose metabolism, but also of the respiration. With respiration correlated PET, blurring does not occur, so it should improve target volume definition. Respiration correlated PET might also be very useful to image nonhomogeneous tumors. A malignant tumor is not a homogeneous mass, but is composed of regions that differ in radioresistance. This intra-tumor heterogeneity can be visualized with PET imaging and opens new therapeutic possibilities for individualized patient treatment. However, the visualization of this intratumor heterogeneity could be distorted if no respiration correlation is performed.

Another consideration when trying to keep PET a quantitative technique, is the attenuation correction. In a CT-PET scanner, the CT information is used for the calculation of the attenuation correction. However, discrepancies between the fast CT and slow PET images could introduce artifacts. We therefore investi- 
gated how an RC-PET should be optimally corrected for attenuation in phantom measurements (Chapter 3) and in simulations based on real RC-CT patient data (Chapter 4). To allow reliable quantification of the PET-signal, attenuation related errors should be minimized. These errors depend on tumor location, tumor size and tumor motion. In all simulated cases, the phased attenuation correction yielded the best results.

The binning of the images was based on the phase of the respiration instead of the amplitude of the respiration. The latter method is more accurate but can lead to gaps in the coronal images, depending on the patient's breathing pattern. Phase binning exhibits no gaps but could generate artifacts due to misbinning ${ }^{5}$. For RC-CT, with a relatively short acquisition time, we seldom see irregular breathing patterns. Therefore we believe that phase binning is an acceptable method of reconstruction. For RC-CT-PET, more research on a large patient scale is needed to quantify errors between the two methods.

To allow the use of (RC-) CT-PET suitable for radiotherapy simulation and dose calculation, a flat carbon table should be used, a moveable laser system and the CT Hounsfield units should be calibrated to electron densities. Although this is an initial investment, in the end it will be more cost effective, since in that case no additional CT for radiotherapy planning has to be performed.

In conclusion, the main reasons for making RC-CT-PET scans are: avoiding a systematic error, avoiding image blurring, and improving auto-delineation procedures since it allows quantification of the PET signal when using phased attenuation correction. In addition, the RC-CT-PET also offers unique possibilities for further research on intra-tumor heterogeneity and correlation of CT-PET with pathology data. This indicated that an RC-CT-PET should be the standard procedure for CT-PET imaging of lung cancer patients.

\section{Repeated imaging during radiotherapy}

The main purpose of repeat imaging during therapy is to see if the GTV and its motion changes during therapy. We used repeated CT-PET and repeated RC-CT scans to investigate these changes. This has the advantage, over in-room imaging, that the diagnostic image quality is preserved. In addition, apart from anatomical information it allows to investigate metabolic changes. The consequence of anatomical changes could be a change in the size of the treatment fields. But maybe in some cases also the field geometry should be adjusted, when critical organs (e.g. the spinal cord) become dose limiting.

We observed for some patients a large increase in tumor volume during therapy. For those patients tumor control might be improved by enlarging the treatment fields. For the patients with decreasing tumor volumes, more normal tissue can be spared with shrinking treatment fields. 
Changes in tumor motion were relatively small, especially when looking at the impact on the internal safety margin, which is only a fraction of the motion (Chapter 5).

The second purpose of repeated PET imaging was to see if we could find a predictive parameter for response early in the treatment and to enable early adjustments, like prescribing a higher total dose, adjusting the fractionation scheme or adding another treatment modality (Chapter 6). With this information we can also investigate if the intra-tumor heterogeneity remains stable during therapy. This information is necessary to develop new treatment options, such as boosting radio-resistant areas within the tumor.

In locally advanced NSCLC, the mediastinal lymph nodes are often involved. Therefore similar knowledge as for the primary tumor is needed for adaptive treatment. When looking at the changes, for the volume as well as for the motion, the clinical impact is relatively small. However, like for tumor motion, there was a large inter-patient variability of nodal motion, even for involved lymph nodes close to the mediastinum (for example: nodal area seven ${ }^{6}$ ). The nodal motion was somewhat smaller than tumor motion. Even within the same patient with multiple involved lymph nodes, differences were observed in nodal motion depending on its anatomical location (Chapter 7). This underscores the need of making an RC-CT not only for the tumor but also for the lymph nodes and to use a patient and nodal specific anisotropic internal margin.

\section{FUTURE PERSPECTIVES}

Non-small cell lung cancer (NSCLC) is a leading cause of cancer mortality in both men and women. Therefore those patients are a big challenge as they have a very high incidence of both local tumor recurrence and distant failure.

One of the first chains in the radiotherapy process is imaging the patient for target definition. A lot of effort has been made to incorporate PET information for treatment planning. A discussed before, this should be replaced by an RC-CTPET but currently no institution uses these scans routinely for all patients. Therefore RC-CT-PET scanning protocols should be optimized and standardized to assure good image quality (i.e. a good signal/noise ratio). This includes investigating the benefits of phased attenuation, regarding quantitative parameters like the $\mathrm{SUV}_{\max }$, on real RC-CT and RC-PET patient data.

At present, no data are yet available on 3D correlation of PET information with the pathologic information. This could give information about the extent of the tumor and can be used as an input for developing auto-delineation protocols. This would reduce further the interobserver variation and would be less time consuming for the radiation oncologist. It will also facilitate multi-institutional studies when a uniform, preferably automated, delineation protocol is available. 
Radiotherapy patients are mostly inoperable and therefore we are currently performing a study to validate RC-CT-PET delineation with pathology in a different, i.e. operable patient population ${ }^{7}$. Another way to tackle this problem is to prospectively investigate the result of autodelineation algorithms by looking at local recurrence patterns.

When resources do not allow to make RC-CT-PET images, another solution is described recently ${ }^{8,9}$. Deep inspiration breath hold (BH) CT-PET imaging looks promising and an increased detection and better characterization of lung lesions compared with a standard clinical CT-PET protocol is feasible. This technique is easy to implement in clinical practice and requires only a minor increase in the examination time. It also reduces breathing artifacts, misregistration, and inaccuracies in SUV measurements. Of course treatment should be performed in the same position which will increase treatment time on the linear accelerator and will be more critical to assure similar patient setup during treatment. Another drawback of this approach is the ability of lung cancer patients to hold their breath during scanning and treatment.

These innovative imaging techniques for FDG-PET scanning could also be extrapolated to other tracers, which visualize different biological characteristics of the tumor.

In the near future we will investigate if the information obtained by repeated CT-PET images, is worthwhile to adapt the treatment fields. Calculations will be done for the dose distribution of the tumor and the surrounding tissues. We also need to look more in depth at the biological variations during therapy and try to answer important questions why $\mathrm{SUV}_{\max }$ changes occurs differently for metabolic responders versus metabolic non-responders. These comparisons will be performed using non-rigid registrations algorithms to minimize deformation artifacts. At all timepoints blood samples were acquired which could give more insight in these tumor dynamics.

Gaining more knowledge of intra-tumor heterogeneity by imaging also implies improving treatment modalities. As the total radiation dose is restricted by virtue of the normal tissue constraints, dose redistribution (higher dose to resistant and lower dose to sensitive areas) within the tumor has a theoretical advantage. This can be done by introducing IMRT (subvolume boosting or dose painting) and delivering a non-homogenous dose to more radioresistant areas within the tumor. Another possibility is to treat the radioresistant zones within the primary tumor with hypofractionation (fraction size of $10 \mathrm{~Gy}-20 \mathrm{~Gy}$ ) followed by radiotherapy to the whole tumor, with more conventional fraction doses.

Currently, most studies and predictive models, for instance to predict outcome or radiation induced normal tissue injury, are based on planned dose distributions. When the theoretical distribution could be replaced by the actual delivered dose, this will improve accuracy in developing these predictive algorithms. The delivered dose can be measured with repeated imaging using an electronic 
portal imaging device (EPID), and research is ongoing of converting the twodimensional EPID information behind the patient to three-dimensional dose distributions within the patient ${ }^{10}$.

\section{REFERENCES}

1. van Herk M. Errors and margins in radiotherapy. Semin Radiat Oncol 2004;14:52-64.

2. Mac Manus M, Hicks RJ, Everitt S. Role of PET-CT in the optimization of thoracic radiotherapy. J Thorac Oncol 2006;1:81-84.

3. De Ruysscher D, Wanders S, van Haren E, et al. Selective mediastinal node irradiation based on FDG-PET scan data in patients with non-small-cell lung cancer: a prospective clinical study. Int J Radiat Oncol Biol Phys 2005;62:988-994.

4. Grills IS, Yan D, Black QC, et al. Clinical implications of defining the gross tumor volume with combination of CT and 18FDG-positron emission tomography in non-small-cell lung cancer. Int J Radiat Oncol Biol Phys 2007;67:709-719.

5. Abdelnour A, Nehmeh S, Pan T, et al. Phase and amplitude binning for 4D-CT. Phys Med Biol 2007;52:3515-3529.

6. Mountain CF, Dresler CM. Regional lymph node classification for lung cancer staging. Chest 1997;111:1718-1723.

7. Stroom JC, Blaauwgeers H, van Baardwijk A, et al. Feasibility of Pathology-correlated Lung Imaging for Accurate Target Definition of Lung Tumors. Int J Radiat Oncol Biol Phys 2007;69:267-275.

8. Meirelles GS, Erdi YE, Nehmeh SA, et al. Deep-Inspiration Breath-Hold PET/CT: Clinical Findings with a New Technique for Detection and Characterization of Thoracic Lesions. $J$ Nucl Med 2007;48:712-719.

9. Nehmeh SA, Erdi YE, Meirelles GS, et al. Deep-inspiration breath-hold PET/CT of the thorax. J Nucl Med 2007;48:22-26.

10. van Elmpt WJ, Nijsten SM, Schiffeleers RF, et al. A Monte Carlo based three-dimensional dose reconstruction method derived from portal dose images. Med Phys 2006;33:2426-2434. 


\section{Su m mary}



Radiotherapy plays an important role in the management of treating lung cancer patients. In this thesis two approaches to improve radiotherapy for patients with lung cancer are investigated. The first approach is to better define the target using novel imaging techniques such as respiration correlated (RC-) CT and respiration correlated CT-PET. These techniques take into account the intra-fraction variations and artifacts due to respiration. In the second approach the target is followed during treatment using repeated (RC-) CT and CT-PET imaging, with the final goal being adaptation of radiotherapy during treatment and tackle interfraction changes.

In Chapter 2 we determined which method of internal target volume (ITV) definition based on a respiration correlated CT (RC-CT) allows optimal tumor coverage. Therefore we acquired both a free breathing $\mathrm{CT}\left(\mathrm{CT}_{\mathrm{fb}}\right)$ and an $\mathrm{RC}-\mathrm{CT}$ scan. The RC-CT scan was used to measure tumor motion and to reconstruct a $\mathrm{CT}$ at 10 phases $\left(\mathrm{CT}_{10 \mathrm{ph}}\right)$, amongst which the half ventilation $\mathrm{CT}\left(\mathrm{CT}_{\mathrm{hv}}\right)$. By averaging the $\mathrm{CT}_{10 \mathrm{ph}}$, a slow $\mathrm{CT}\left(\mathrm{CT}_{\text {slow }}\right)$ was reconstructed. Based on those scans ITVs were delineated and treatments were planned, where for the $\mathrm{ITV}_{\mathrm{hv}}$ an internal margin (margin for tumor motion) of (motion amplitude)/ 4 was used. The treatment plans for the ITVs were projected on the 10 respiration phases and subsequently the absorbed dose was calculated and averaged over the ten phases to estimate the actual CTV coverage. This was an in silico (plannings) study based on real RC-CT patient data. The $\mathrm{CT}_{\mathrm{hv}}$ seems to be the optimal dataset for delineation, using an adequate anisotropic internal margin.

Since accurate knowledge of the position of the tumor and the surrounding tissues is needed for radiation treatment planning it is important to improve CTPET image acquisition. In Chapter 3 we evaluated the potential to improve image acquisition using phased attenuation correction in respiration correlated CTPET, where data of both modalities was binned retrospectively. After collection of the raw CT data, list-mode PET data, and the respiratory signal CT-PET images were binned to ten phases with help of in-house built software. Each PET phase was corrected for attenuation with CT data of the corresponding phase. For comparison, the attenuation correction was also performed with nonrespiration correlated (non-RC) CT data. With a standard, non-RC CT-PET scan, the volume was underestimated by as much as $46 \%$ in CT and the PET volume was overestimated to $370 \%$ whereas the volumes with RC-CT-PET scanning had small deviations from the actual volume. Evaluation of the maximum activity concentration showed a clear displacement in the images with non-RC attenuation correction. In this phantom study we have shown that a combination of respiration correlated CT-PET scanning with application of phased attenuation correction can improve the imaging of moving objects and 
can lead to improved volume estimation and a more precise localization and quantification of the activity.

To quantify the attenuation errors and identify the parameters associated with them, we performed a simulation based on respiratory-gated CT studies of five lung cancer patients in Chapter 4. The CT series were converted to $511-\mathrm{keV}$ attenuation maps which were forward-projected and exponentiated to form sinograms of PET attenuation factors at each phase of respiration. The CT images were also segmented to form a PET object, moving with the same motion as the CT series. In the moving PET object, spherical 20-mm mobile tumors were created in the vicinity of the dome of the liver, and immobile 20 -mm tumors in the mid chest region. The moving PET objects were forward-projected and attenuated, then reconstructed in several ways: phase-matched PET and CT; gated PET with ungated CT; ungated PET with gated CT; and conventional PET. Phase-matched gated PET and CT gave essentially perfect PET reconstructions in the simulation. Gated PET with ungated CT gave tumors of the correct shape, but recovery was too large by an amount that depended on the extent of the motion, as much as $90 \%$ for mobile tumors and $60 \%$ for immobile tumors. Gated CT with ungated PET resulted in blurred tumors and caused the recovery errors between $-50 \%$ and $+75 \%$. Recovery in clinical scans would be $0 \%$ to $20 \%$ lower than stated. Quantitative PET imaging near the diaphragm requires proper matching of attenuation information to the emission information.

Chapter 5 deals with the change in tumor volume, motion and breathing frequency during a course of radiotherapy, for locally advanced non-small cell lung cancer. Twenty-three patients underwent CT-PET and Respiration Correlated CT scans prior to treatment, which was repeated in the first and second week following the start of radiotherapy. Patients were treated with an accelerated fractionation schedule with a total tumor dose depending on pre-set dose constraints for the lungs and the spinal cord. A striking heterogeneity of tumor volume changes was observed at all time points. In some patients the volume decreased $>30 \%$ in others the volume increased $>30 \%$ but for the majority of patients the tumor volume only changed slightly. No significant changes in average tumor motion or breathing frequencies were seen during treatment. These results underscore the need for repeated imaging during the course of radiotherapy. However, the changes in tumor motion are small, which indicates that repeated respiration correlated CT does not appear to be necessary for the primary tumor.

${ }^{18}$ F-fluoro-2-deoxy-glucose (FDG) uptake on PET scan is a prognostic factor for outcome in NSCLC. We investigated changes in FDG uptake during fractionated radiotherapy in relation to metabolic response with the ultimate aim to 
adapt treatment according to early response (chapter 6). The same patient population of the previous chapter was examined. Almost all patients had a 70 days post treatment CT-PET scan. Changes in maximal standardized uptake value $\left(\mathrm{SUV}_{\max }\right)$ were described. The most striking result was the large intraindividual heterogeneity in the evolution of $\mathrm{SUV}_{\max }$. Different time trends were shown for responders (no change during radiotherapy) and non-responders (48\% increase during first week). Non-responders had a higher $\mathrm{SUV}_{\max }$ on all time points investigated. Time trends in $\mathrm{SUV}_{\max }$ showed a large intra-individual heterogeneity and different patterns for metabolic responders and nonresponders. These new findings may reflect intrinsic tumor characteristics and might finally be useful to adapt treatment.

Chapter 7 describes the time trends in nodal CT volume and nodal motion. Twelve patients, with a total of 22 positive nodes, underwent a CT-PET scan prior to treatment, as well as in the first and second week following start of radiotherapy. Volume changes could be measured for all nodes. For 21 nodes, the motion was measured based on a respiration correlated CT (RC-CT) scan. Repeated RC-CT scans were available for 11 nodes to evaluate the change in motion. In $27 \%$ patients the nodal volume increased $>30 \%$ while in $14 \%$ the volume decreased $>30 \%$. These changes had limited clinical impact, since volumes and hence volume changes were small and on average, the nodal volume did not change significantly. The average motion of the nodal areas was initially $5.6 \mathrm{~mm} \pm 2.8 \mathrm{~mm}$. This motion decreased slightly during therapy but not statistically significant. Large inter-patient and inter-nodal motion differences were observed. However, because of the large inter patient variability of nodal motion prior to treatment, internal margins for nodal areas should be calculated before radiotherapy using RC-CT, such that the margins can be applied for individual patients. Repeated imaging of the nodes seems however to be of limited use, as the observed individual changes in nodal volume and motion tend to fall within the commonly applied margins. 



\section{Samenvatting}



Radiotherapie speelt een belangrijke rol in de behandeling van longkanker patienten. In dit proefschrift worden twee benaderingen onderzocht om de radiotherapie te verbeteren voor deze patiënten. De eerste benadering is het beter definieren van het doelvolume, gebruik makend van vernieuwende beeldvormingstechnieken zoals ademhalingsgecorreleerde CT (RC-CT) en ademhalingsgecorreleerde CT-PET (RC-CT-PET). Deze technieken houden rekening met de intra-fractie veranderingen en artefacts te wijten aan de ademhaling. In de tweede benadering wordt het doelvolume gevolgd tijdens de behandeling gebruik makend van herhaalde (RC)-CT en CT-PET beeldvorming, met het uiteindelijke doel om de radiotherapiebehandeling aan te passen tijdens de behandeling en om de inter-fractie veranderingen aan te pakken.

In Hoofdstuk 2 hebben we bepaald welke methode om het interne doelvolume (ITV) te bepalen, gebaseerd op een RC-CT, een optimale dekking van de tumor toelaat. Daarom hebben we zowel een gewone 'vrije ademhalings' CT $\left(\mathrm{CT}_{\mathrm{fb}}\right)$ en een RC-CT scan gemaakt. De RC-CT scan werd gebruikt om de tumorbeweging te meten en om een 10 fasen $\mathrm{CT}$ te reconstrueren $\left(\mathrm{CT}_{10 \mathrm{ph}}\right)$, waaronder de halfventilatie $\mathrm{CT}\left(\mathrm{CT}_{\mathrm{hv}}\right)$. Door de $\mathrm{CT}_{10 \mathrm{ph}}$ te middelen werd een 'trage' $\mathrm{CT}$ scan bekomen $\left(\mathrm{CT}_{\text {slow }}\right)$. Op basis van al deze scans werden ITVs ingetekend en behandelingen berekend, waarbij we voor het $\mathrm{ITV}_{\mathrm{hv}}$ een interne marge (marge voor de tumor beweging) van bewegingsamplitude gedeeld door vier werd gebruikt. Al de behandelplannen voor de verschillende ITVs werden geprojecteerd op de 10 ademhalingsfasen en vervolgens werd de geabsorbeerde dosis berekend over alle 10 fasen om de reële dekking van de tumor in te schatten. Dit was een planningstudie gebaseerd op echte RC-CT patiënt gegevens. De $\mathrm{CT}_{\mathrm{hv}}$ lijkt de optimale dataset te zijn voor intekening, gebruik makend van een voldoende grote anisotrope interne marge.

Nauwkeurige kennis van de positie van de tumor en het omliggende weefsel is nodig voor de radiotherapiebehandelingsplanning en het is belangrijk om de CT-PET beeldvorming te verbeteren. In Hoofdstuk 3 hebben we de mogelijkheid geevalueerd om de acquisitie te verbeteren door gebruik te maken van gefaseerde verzwakkingscorrectie in RC-CT-PET, waar beide modaliteiten retrospectief opgedeeld zijn. Na het verzamelen van de ruwe CT gegevens, de listmode PET gegevens en het ademhalingssignaal, werden de CT-PET beelden opgedeeld in 10 fasen met behulp van zelf geschreven software. Elke PET fase was gecorrigeerd voor verzwakking met de CT gegevens van de overeenkomstige fase. Ter vergelijking was ook de verzwakkingscorrectie uitgevoerd met CT data die niet ademhalingsgecorreleerd was. Met een standaard, niet ademhalingsgecorreleerde CT-PET scan werd het volume onderschat tot $46 \%$ in de CT beelden en overschat tot $370 \%$ voor het PET volume, terwijl met de RC-CTPET de volumes konden bepaald worden met kleine afwijkingen ten opzichte 
van het reële volume. Het evalueren van de maximale activiteitsconcentratie liet een duidelijke verschuiving zien in de niet ademhalingsgecorreleerde beelden van dit maximum. In deze fantoomstudie hebben we laten zien dat een combinatie van RC-CT-PET beeldvorming met toepassing van gefaseerde verzwakkingscorrectie, de beeldvorming kan verbeteren van bewegende voorwerpen en kan leiden tot een verbeterde volumebepaling en een meer precieze lokalisatie en kwantificatie van de activiteit.

Om de verzwakkingsfouten te kwantificeren en de parameters te identificeren verbonden met deze fouten, hebben we een simulatie uitgevoerd gebaseerd op ademhalingsgecorreleerde CT studies van vijf longkanker patiënten in Hoofdstuk 4. De CT werd geconverteerd naar 511-keV verzwakkingsmappen welke voorwaarts geprojecteerd en geexponentieerd werden om sinogrammen te vormen van PET verzwakkingsfactoren voor elke fase van de ademhaling. De CT beelden werden ook gesegmenteerd om een PET object te vormen met dezelfde beweging als de CT data. In de bewegende PET objecten werden bolvormige $20 \mathrm{~mm}$ bewegende tumoren aangemaakt in de buurt van de top van de lever en niet bewegende tumoren in de buurt van het midden van de borstkas. De bewegende PET objecten werden voorwaarts geprojecteerd en verzwakt en daarna op verschillende manieren gereconstrueerd: gefaseerde PET en CT, gated PET met ungated CT, ungated PET met gated CT en conventionele PET. De gefaseerde gated PET en CT gaf perfecte reconstructies in de simulatie. Gated PET met ungated CT gaf tumoren met de correcte vorm maar de recuperatie van de maximale activiteit was te groot, afhankelijk van de grootte van tumorbeweging, tot $90 \%$ voor bewegende tumoren en $60 \%$ voor niet bewegende tumoren. Gated CT met ungated PET resulteerde in onscherpe tumoren en veroorzaakte fouten in recuperatie tussen $-50 \%$ en $+75 \%$. In klinische scans zou de recuperatie $0 \%$ tot $20 \%$ lager uitvallen. Kwantitatieve PET beeldvorming dichtbij het diafragma vereist gepaste matching van verzwakkingsinformatie voor de emissie informatie.

Hoofdstuk 5 gaat over de verandering in tumorvolume, tumorbeweging en ademhalingsfrekwentie tijdens een radiotherapiebehandeling voor patiënten met uitgebreid niet-kleincellig longkanker. Drieëntwintig patiënten kregen een CTPET en RC-CT scan voor de behandeling die herhaald werd in de eerste en tweede week volgende op de start van de radiotherapie. De patiënten werden behandeld met een versneld fractionatieschema met een totale tumor dosis afhankelijk van voorgenomen dosisbeperkingen voor de longen en het ruggemerg. Een opvallende heterogeniteit van de tumorvolume veranderingen werd waargenomen op alle tijdstippen. Voor sommige patiënten werd het volume meer dan 30\% kleiner, voor andere werd het meer dan 30\% groter maar voor de meerderheid van de patiënten veranderde het volume slechts gering. Geen signi- 
ficante veranderingen in gemiddelde tumorbeweging of ademhalingsfrekwentie werd waargenomen tijdens de behandeling. Deze resultaten onderstrepen de noodzaak om herhaalde beeldvorming tijdens de radiotherapiebehandeling uit te voeren. De veranderingen in tumorbeweging waren klein, daarom lijken herhaalde ademhalingsgecorreleerde CT scans niet noodzakelijk voor de primaire tumor.

${ }^{18}$ F-fluoro-2-deoxy-glucose (FDG) opname op PET beelden is een voorspellende factor voor de uitkomst voor patiënten met niet-kleincellig longkanker. We hebben de veranderingen onderzocht in deze FDG opname tijdens gefractioneerde radiotherapie in relatie tot de metabole respons met het uiteindelijke doel de behandeling aan te passen volgens de vroege respons (Hoofdstuk 6). Dezelfde patiëntpopulatie als in het vorige hoofdstuk werd onderzocht. Bijna alle patiënten hadden ook een CT-PET 70 dagen na het einde van de behandeling. Veranderingen in de maximale gestandaardizeerde opname waarde $\left(\mathrm{SUV}_{\max }\right)$ zijn beschreven. Het meest opvallende resultaat was de grote heterogeniteit in evolutie van de $\mathrm{SUV}_{\max }$ tussen de patiënten. Verschillende tijd trends werden gezien voor responders (geen verandering tijdens radiotherapie) en voor nietresponders ( $48 \%$ toename in de eerste week). Niet-responders hadden op ieder onderzocht tijdspunt een hogere $\mathrm{SUV}_{\max }$. Tijd trends in $\mathrm{SUV}_{\max }$ lieten een grote intra-individuele heterogeniteit en verschillende patronen voor metabole responders en niet-responders zien. Deze nieuwe bevindingen weerspiegelen mogelijks intrinsieke tumoreigenschappen en kunnen uiteindelijk bruikbaar zijn om de behandeling aan te passen.

Hoofdstuk 7 beschrijft de tijd trends in kliervolume en klierbeweging op de CT scan. Twaalf patiënten, met in totaal 22 positieve klieren, ondergingen herhaalde CT-PET scans voor de behandeling en de eerste en tweede week na de start van de radiotherapie. Volumeveranderingen konden gemeten worden voor alle klieren. Voor 21 klieren was de beweging gemeten op basis van een RC-CT scan. Herhaalde RC-CT scans waren beschikbaar voor 11 klieren om de bewegingsveranderingen te evalueren. In $27 \%$ van de patiënten nam het kliervolume toe met meer dan $30 \%$ terwijl in $14 \%$ het volume daalde meer dan $30 \%$. Deze volumeveranderingen hebben echter weinig klinisch impact gezien de volumes en dus ook de volumeveranderingen klein zijn en gemiddeld veranderde het kliervolume niet significant. De gemiddelde beweging van de klierregio's was initieel $5.6 \mathrm{~mm} \pm 2.8 \mathrm{~mm}$. Deze beweging verminderde lichtjes tijdens de radiotherapiebehandeling maar niet statistich significant. Grote verschillen in beweging tussen de patiënten en tussen klierregio's binnen dezelfde patiënt werden waargenomen. Vanwege de grote variabiliteit in klierbeweging tussen de patiënten onderling, moeten de interne marges voor deze klierregio's individueel berekend worden voor de start van de behandeling gebruik makend van een RC- 
CT. Herhaalde beeldvorming voor de klieren lijkt echter van weinig nut, gezien de geobserveerde individuele veranderingen in kliervolume en klierbeweging lijkt omvat te zijn binnen de huidige toegepaste marges. 


\section{Da nkwoord}



Het is altijd een risico om een dankwoord te schrijven omdat de kans bestaat dat je iemand, uiteraard niet moedwillig, kan vergeten. Vandaar dat ik wil beginnen met iedereen te bedanken die op welke manier dan ook heeft bijgedragen tot het welslagen van dit proefschrift. Hier horen natuurlijk ook de patiënten bij, die door hun deelname aan studies, het ons mogelijk maken om klinisch onderzoek te verrichten.

Bijna vier jaar geleden overtuigde André Minken en Philippe Lambin mij om als klinisch fysicus te komen werken in Maastricht. Een bijkomende aantrekkingskracht was de mogelijkheid om te promoveren, een optie die ik op dat moment als interessant beschouwde maar niet echt als een meerwaarde. $\mathrm{Nu}$, na het volmaken van dit proefschrift, zie ik het als een echte verrijking en niet alleen voor mijn 'carrière'.

Philippe Lambin, promotor. Beste Philippe, bedankt voor je aanmoedigingen en kritische bijdragen. Het is vaak onbegrijpelijk, met je drukke agenda, hoe snel je opmerkingen op manuscripten terug kwamen.

Dirk De Ruysscher, co-promotor. Beste Dirk, zonder jouw onuitputtelijke ideeen zou deze promotie nooit zo vlot verlopen zijn. Ik sta nog altijd versteld van de tijd die je kunt vrijmaken voor iedereen en ik apprecieer het enorm dat een (klinische) vraag nooit te veel is. Het enige probleem is dat een antwoord van 5 minuutjes al vlug eindigde in discussies van een paar uur en resulteerde in extra werk voor de komende weken.

André Dekker, co-promotor. André bedankt voor je inzichtelijke opmerkingen en aanbevelingen op de manuscripten, vaak van de lastigste om te beantwoorden, en het efficiënt sturen van mijn onderzoek. Je hebt ook voor een vlotte start van dit proefschrift gezorgd doordat ik een deel van je eigen onderzoek kon verder zetten.

André Minken, mijn eerste begeleider. Beste André, je hebt me uiteindelijk overtuigd om te starten met de promotie. Hiervoor schiep je ook de ideale omstandigheden door mij twee dagen per week onderzoekstijd te geven. De grote lijnen van dit proefschrift zijn ook nog onder jouw supervisie uitgezet.

De promotiecommissie wil ik bedanken voor alle tijd gespendeerd aan het lezen en beoordelen van dit proefschrift.

Beste Angela, het gezamenlijk uitvoeren en publiceren van ons onderzoek was zeer aangenaam. Ik kon ook steeds bij je terecht voor mijn (eenvoudige) klini- 
sche vragen, zoals 'Waar zit de tumor?'. En samen probeerden we de waterval van Dirk's ideeën te trotseren. Gelukkig stond ik hierbij niet alleen.

Liesbeth Boersma, jou wil ik bedanken voor je vaak zeer terechte reviews van de manuscripten, zowel voor de grote lijnen maar zeker ook voor alle komma's, punten en afkortingen. Het kritisch nalezen van dit proefschrift tussen je drukke bezigheden waardeer ik enorm.

Verder wil ik ook alle andere co-auteurs bedanken voor het publiceerbaar maken van de manuscripten. Bedankt Rinus, Jeroen, Cathryn, Jim, Marije, Marinus, Ruud, Norbert en Klaus.

Mijn paranimfen, Hugo en Peter, bedankt voor de steun en plezier bij het beëindigen van het onderzoek.

Uiteraard wil ik ook al mijn rechtstreekse (ex) collega klinisch fysici (i.o.) bedanken voor het overnemen van de klinische taken tijdens een onderzoeksweek, congres of één of ander research bezoek. Bedankt Lars, Bas, Peter, Michel, Robert, Martina, Martijn, Erik en Ramon.

Ook de rest van de hele KFG groep bedankt voor de aangename momenten tijdens lunchpauzes, commissioning metingen, avondjes uit,...

Uiteindelijk zijn alle scans en behandelingen dagelijks uitgevoerd door de laboranten en ingepland door de ondersteuning. Zonder deze, vaak onzichtbare inzet achter de schermen, kan er geen klinisch onderzoek verricht worden. Bedankt!

Bianca, bedankt voor het ontwerpen van de cover in je avonduren, een kopzorg minder tijdens de voorbereiding.

De secretariële ondersteuning is uiteraard onmisbaar in het kluwen van dokumenten die ingediend moeten worden en voor het verzenden van alle proefschriften. Bedankt Rian, Simone, Sonia, Marlene en Nelly.

Uiteindelijk wil ik ook familie en vrienden bedanken. Deze promotie was in de afgelopen drie jaren een continu gegeven en hopelijk kan ik nu eindelijk mijn hoofd 's avonds en in het weekend leegmaken en meer tijd voor hen vrijmaken.

Ann en Janne, bedankt voor jullie geduld en vanaf nu is je man en je papa er terug voor $100 \%$. 
Curriculum Vitae 

Geert Bosmans werd geboren op 1 juni 1973 in Hasselt (België) en groeide op in Zonhoven. Zowel lagere als middelbare school doorliep hij in Hasselt en behaalde in 1991 het ASO diploma (Wiskunde-Wetenschappen).

Hierna ging hij natuurkunde (fysica) studeren, de eerste twee kandidaatsjaren aan het toenmalige Limburgs Universitair Centrum (nu Universiteit Hasselt) te Diepenbeek. In 1995 behaalde hij zijn licentiaatsdiploma natuurkunde aan de Katholieke Universiteit Leuven met een thesis in het vakgebied van halfgeleiderfysica.

Vermits de zuivere fysica hem niet kon bekoren volgde hij nog een bijkomende opleiding tot klinisch fysicus, waarvoor het diploma 'aanvullende studies biomedische en klinische ingenieurstechnieken optie medische fysica' gehaald moest worden. Hierin was een éénjarige stageperiode omvat die op de radiotherapie afdeling van het UZ Leuven is gebeurd en waarbij ook een thesis over amorf silicium EPID's werd gemaakt o.l.v. Prof. dr. Jan van Dam.

$\mathrm{Na}$ een korte werkervaring in de ICT (Cap Gemini Belgium) is hij sinds begin 1998 continu aan de slag als klinisch fysicus radiotherapie. Eerst in het Heilig Hart Ziekenhuis te Roeselare dan in het AZ-VUB te Brussel en tenslotte in het Limburgs Oncologisch Centrum te Hasselt.

De nederlandse lokroep werd echter te groot en sinds oktober 2004 is hij werkzaam in Maastro Clinic te Maastricht. Na een verkorte opleiding niveau 3 stralingsbescherming is hij vanaf januari 2005 geregistreerd als klinisch fysicus radiotherapie in Nederland.

Zijn voornaamste aandachtsgebieden zijn CT-PET beeldvorming, stereotactische bestralingen en patiëntveiligheid.

Hij is getrouwd met Ann Hendrickx en hebben samen één dochtertje, Janne. 

Publications | 151

Publications 

Articles

A. van Baardwijk, B. Baumert, G. Bosmans, M. van Kroonenburgh, S. Stroobants, V. Gregoire, Ph. Lambin, D. De Ruysscher. The current status of FDG-PET in tumour volume definition in radiotherapy treatment planning. Cancer Treat Rev 2006;32(4):245-260.

C. Nagel, G. Bosmans, A. Dekker, M. Öllers, D. De Ruysscher, Ph. Lambin, A. Minken, N. Lang, K. Schäfers. Phased attenuation correction in respiration correlated CT/PET. Med Phys 2006;33(6):1840-1847.

G. Bosmans, J. Buijssen, A. Dekker, M. Velders, L. Boersma, D. De Ruysscher, A. Minken, Ph. Lambin. An "in silico" clinical trial comparing free breathing, slow and respiratory correlated computed tomography in lung cancer patients. Radiother Oncol 2006;81(1):73-80.

G. Bosmans, A. van Baardwijk, A. Dekker, M. Öllers, L. Boersma, A. Minken, $\mathrm{Ph}$. Lambin, D. De Ruysscher. Intra-patient variability of tumor volume and tumor motion during conventionally fractionated radiotherapy for locally advanced non-small cell lung cancer. A Prospective Clinical Study. Int J Radiat Oncol Biol Phys 2006;66(3):748-753.

A. van Baardwijk, G. Bosmans, A. Dekker, M. van Kroonenburgh, L. Boersma, S. Wanders, M. Öllers, A. Minken, Ph. Lambin, D. De Ruysscher. Time trends in the maximal uptake of FDG on PET scan during thoracic radiotherapy in relation to metabolic response. A prospective study in locally advanced non-small cell lung cancer patients. Radiother Oncol 2007;82(2):145-152.

A. van Baardwijk, G. Bosmans, L. Boersma, J. Buijsen, S. Wanders, M. Hochstenbach, R.J. van Suylen, A. Dekker, C. Dehing-Oberije, R. Houben, S. Bentzen, M. van Kroonenburgh, Ph. Lambin, D. De Ruysscher. PET-CT scan based autocontouring in non-small cell lung cancer correlates with pathology and reduces interobserver variability in the delineation of the primary tumor and involved nodal volumes. Int J Radiat Oncol Biol Phys 2007;68(3):771-778.

G. Bosmans, A. van Baardwijk, A. Dekker, M. Öllers, S. Wanders, L. Boersma, $\mathrm{Ph}$. Lambin, D. De Ruysscher. Time trends in nodal CT colume and nodal motion during radiotherapy for patients with stage III non-small cell lung cancer. Int J Radiat Oncol Biol 2007; accepted for publication. 
G. Janssens, J. Orban de Xivry, G. Bosmans, M. De Craene, A. Dekker, J. Buijsen, A. van Baardwijk, D. De Ruysscher, B. Macq, Ph. Lambin. Tumor delineation and cumulative dose computation in radiotherapy based on deformable registration of Respiratory Correlated CT images of lung cancer patients. Radiother Oncol 2007; accepted for publication.

J. Hamill, G. Bosmans, A. Dekker. Respiratory-Gated CT as a Tool for the Simulation of Breathing Artifacts in PET and PET/CT. Med Phys 2007; accepted for publication.

\section{Published abstracts}

B. Reymen, A. Maes, K. Bamps, G. Bosmans, E. Bressers, P. Bulens, M. Brosens, Y. Staelens. 3D-TPS and IMRT significantly decreases the hot-spots in breast radiotherapy. Radiother Oncol 2004;73(S1):S335.

A. Dekker, G. Bosmans, M. Öllers, L. Boersma, D. De Ruysscher, A. Minken, $\mathrm{Ph}$. Lambin. Respiratory Correlated CT: Acceptance and Clinical Introduction. Med Phys 2005;32(6):1905.

C. Nagel, A. Dekker, G. Bosmans, M. Öllers, A. Minken, Ph. Lambin, K. Schäfers, N. Lang. Phased attenuation Correction in Respiration Correlated PET/CT. Med Phys 2005;32(6):1913.

A. Dekker, G. Bosmans, J. Buijsen, M. Velders, L. Boersma, D. De Ruysscher, R. Wanders, A. Minken, Ph. Lambin. Comparison between Free Breathing, Slow and Respiratory Correlated CT in Radiation Treatment Planning for Lung Cancer Patients. Med Phys 2005;32(6):1945.

G. Bosmans, J. Buijssen, A. Dekker, M. Velders, L. Boersma, D. De Ruysscher, A. Minken, Ph. Lambin. An "in silico" clinical trial comparing free breathing, slow and respiratory correlated computed tomography in lung cancer patients. Radiother Oncol 2005;77(S2):S92.

G. Bosmans, C. Nagel, A. Dekker, M. Öllers, A. Minken, Ph. Lambin, K. Schäfers, N. Lang. Phased attenuation correction in respiration correlated PET/CT. Radiother Oncol 2005;77(S2):S166.

A. Theelen, J. Martens. G. Bosmans, I. Rutten, H. Beijer, A. Minken, R. Houben, Ph. Lambin, B.G. Baumert. Relocatable fixation systems in intracranial stereotactic radiotherapy: Evaluation by serial CT scans and patient acceptance. Radiother Oncol 2006;81(S1):S49. 
A. van Baardwijk, G. Bosmans, A. Dekker, M. van Kroonenburgh, L. Boersma, S. Wanders, M. Öllers, A. Minken, Ph. Lambin, D. De Ruysscher. Changes in the maximal uptake of FDG on PET scan during radiotherapy in locally advanced non-small cell lung cancer (NSCLC) patients: a correlation with metabolic response. Radiother Oncol 2006;81(S1):S214.

B. Baumert, B.Vanhauten, G. Bosmans, S. Abdulfatah, R. Houben, R. Debougnoux, M. van Kroonenburgh, Ph. Lambin. Prediction of survival in patients with a glioblastoma based on SUV determination before radiotherapy. Radiother Oncol 2006;81(S1):S219.

G. Janssens, J. Orban de Xivry, G. Bosmans, J. Buijsen, M. Decraene, A. Dekker, A. van Baardwijk, B. Macq, Ph. Lambin. Semi-automatic delineation of moving lung tumours using deformation fields based on respiration correlated CT : improvement of the internal target volume definition. Radiother Oncol 2006;81(S1):S247.

J. Orban de Xivry, G. Janssens, G. Bosmans, J. Buijsen, M. Decraene, A. Dekker, A. van Baardwijk, B. Macq, Ph. Lambin. Deformation of planned dose distribution to track delivered dose to Non Small Cell Lung Cancer (NSCLC) patients. Radiother Oncol 2006;81(S1):S255.

A. van Baardwijk, G. Bosmans, J. Buijsen, L. Boersma, R. Wanders, M. Hochstenbach, M. van Kroonenburgh, C. Dehing-Oberije, Ph. Lambin, D. De Ruysscher. FDG-PET based automatic delineation correlates with pathology and reduces interobserver variability in the delineation of tumour and nodal volumes in non-small cell lung cancer (NSCLC). Radiother Oncol 2006;81(S1):S347.

H. Aerts, G. Bosmans, A. van Baardwijk, A. Dekker, M. Öllers, Ph. Lambin, D. De Ruysscher. Time trends in the heterogeneity of FDG uptake, based on SUV contours, during a course of radical radiotherapy in NSCLC. Radiother Oncol 2006;81(S1):S468

G. Bosmans, C. Nagel, I. Potargent, A. Dekker, M. Öllers, D. De Ruysscher, A. Minken, Ph. Lambin. Clinical introduction of Respiration Correlated (4D) CT/PET scanning. Radiother Oncol 2006;81(S1):S512.

A. Dekker, G. Bosmans, A. van Baardwijk, M. Öllers, L. Boersma, A. Minken, $\mathrm{Ph}$. Lambin, D. De Ruysscher. Intra-patient variability of tumor volume and tumor motion during radiotherapy for non-small cell lung cancer. Radiother Oncol 2006;81(S1):S540. 
C. Offermann-Wulms, D. Emans, G. Bosmans, A. Dekker, Ph. Lambin, A. Minken, D. De Ruysscher. Boosting FDG-positive tumour sub-volumes in patients with non-small cell lung cancer (NSCLC): a planning study using IMRT. Radiother Oncol 2006;81(S1):S778.

M. Engelsman, A. Driesen, R Vliegen, B. Vanhauten, D. De Ruysscher, G. Bosmans, T. Eiland, A. van Baardwijk,, R. Beets-Tan, Ph. Lambin, G. Lammering. Tumor response prediction by use of a combined FDG-PET-CT simulator before and after radiochemotherapy in rectal cancer patients. Radiother Oncol 2006;81(S1):S780.

A. van Baardwijk, G. Bosmans, A. Dekker, M. van Kroonenburgh, L. Boersma, S. Wanders, M. Öllers, A. Minken, Ph. Lambin, D. De Ruysscher. Time Trends in the Maximal Uptake of FDG on PET Scan During Thoracic Radiotherapy. A Prospective Study in Locally Advanced Non-Small Cell Lung Cancer (NSCLC) Patients. Int J Radiat Oncol Biol Phys 2006;66-3(S1);S25.

B.G. Baumert, B. Vanhauten, S.B. Abdulfatah, R. Houben, R. DebougnouxHuppertz, M. van Kroonenburgh, G. Bosmans, Ph. Lambin. Combined 18FDG PET-CT Before Radiotherapy May Predict Survival in Patients With a Glioblastoma. Int J Radiat Oncol Biol Phys, 2006;66-3(S1);S197-S198.

G. Bosmans, H. Aerts, A. van Baardwijk, A. Dekker, S. Wanders, L. Boersma, $\mathrm{Ph}$. Lambin, D. De Ruysscher. FDG-PET allows identification of radioresistant areas within the tumor during and after radiation treatment of NSCLC. $J$ Thorac Oncol 2007;2(8)S4:S411-S412.

A. van Baardwijk, G. Bosmans, L. Boersma, S. Wanders, J. Vernooy, A. Mandic Havelka, Ph. Lambin, J. Cohen-Tervaert, D. De Ruysscher. Changes in plasma levels of cytokines during fractionated radiotherapy of non-small cell lung cancer (NSCLC): the relation with metabolic response and toxicity $J$ Thorac Oncol 2007;2(8)S4:S436-S437.

G. Bosmans, A. van Baardwijk,A. Dekker, S. Wanders, L. Boersma, Ph. Lambin, D. De Ruysscher. Time trends in nodal CT volume and nodal motion during radiotherapy for patients with stage III non-small cell lung cancer. $J$ Thorac Oncol 2007;2(8)S4:S473-S474.

A. Dekker, G. Bosmans, A. van Baardwijk, M. Öllers, Ph. Lambin, D. De Ruysscher. The role of PET/CT in delineation, treatment and follow-up in lung cancer. Radiother Oncol 2007;84(S1):S36. 
A. Houben, H. Aerts, G. Bosmans, C. Duisters, D. Emans, Ph. Lambin, A. Dekker, D. De Ruysscher. Towards a better prediction of radiation induced lung damage (RILD). Radiother Oncol 2007;84(S1):S160.

T. Rosario, M. Öllers, G. Bosmans, Ph. Lambin, A. Dekker. Quantitative evaluation of image quality of respiration correlated PET for patients with NSCLC. Radiother Oncol 2007;84(S1):S176.

M. Janssen, H. Aerts, M. Öllers, G. Bosmans, J. Lee, G. Lammering, A. Dekker, Ph. Lambin, D. De Ruysscher. Tumor delineation based on the time activity curve differences assessed with dynamic PET. Radiother Oncol 2007;84(S1):S181.

G. Bosmans, L. Boersma, A. van Baardwijk, A. Dekker, M. Öllers, S. Wanders, Ph. Lambin and D. De Ruysscher. Time Trends in Nodal CT Volume and Nodal Motion During Radiotherapy for Patients With Stage III Non-Small Cell Lung Cancer Int J Radiat Oncol Biol Phys 2007;69-3(S1);S490-S491. 


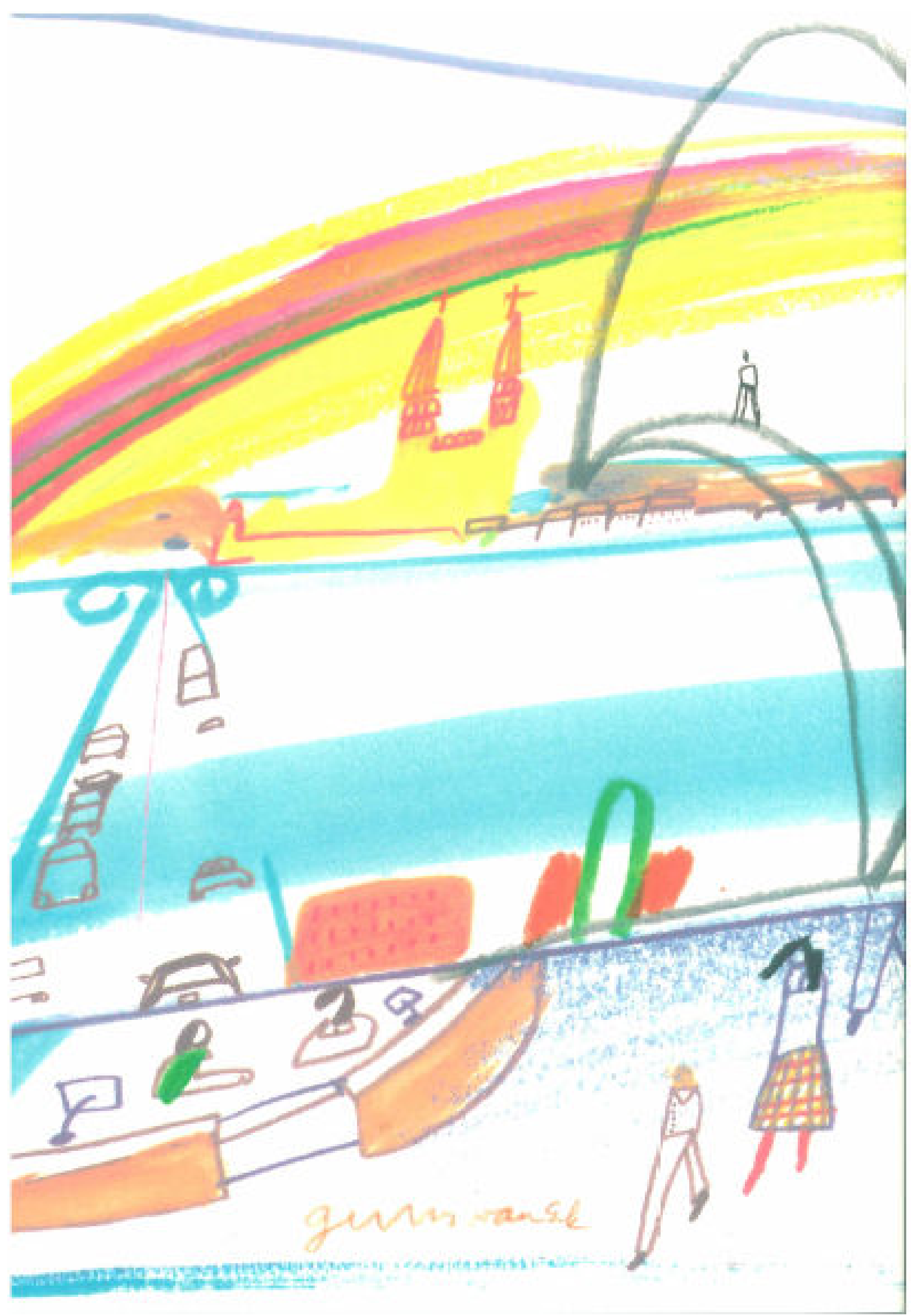

